\title{
A Four-Noded Quadrilateral Element for Composite Laminated Plates/Shells using the Refined Zigzag Theory
}

\author{
A. Eijo \\ E. Oñate \\ S. Oller
}




\section{A Four-Noded Quadrilateral Element for Composite Laminated Plates/Shells using the Refined Zigzag Theory}
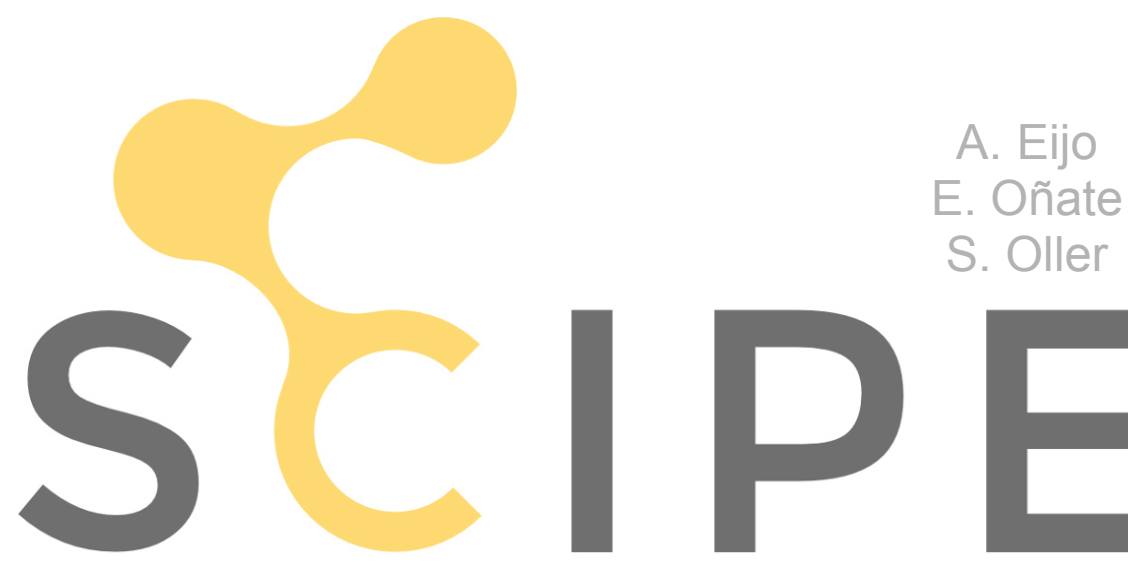

Register for free at https//www.scipedia.com to download the version without the watermark Publication CIMNE №-378, June 2012 


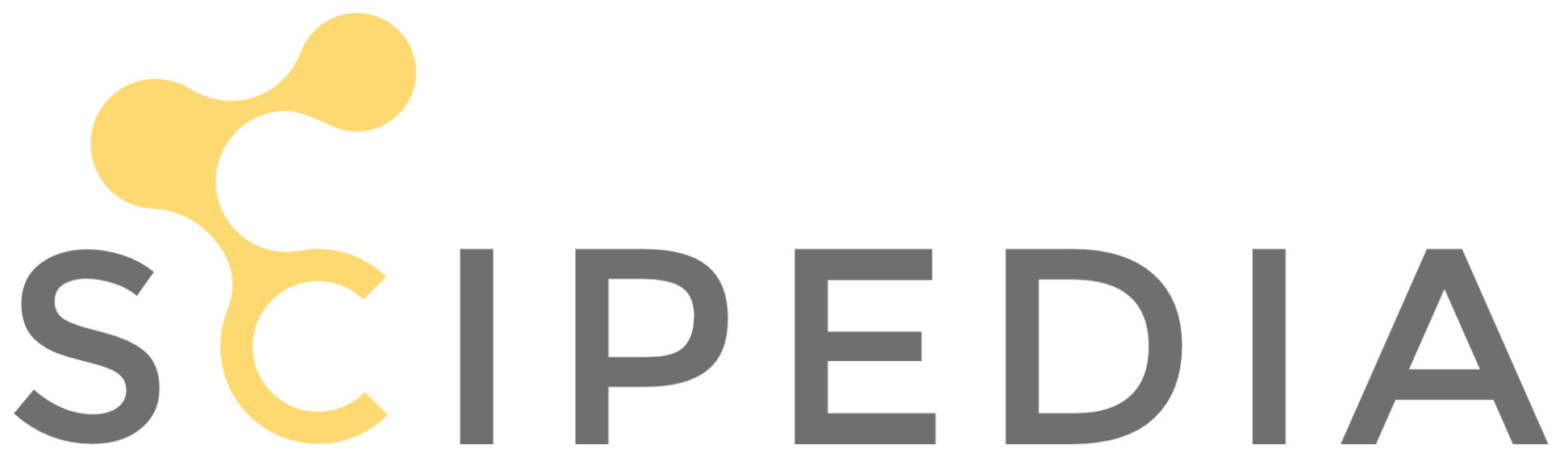

Register for free at https//www.scipedia.com to download the version without the watermark 


\title{
A FOUR-NODED QUADRILATERAL ELEMENT FOR COMPOSITE LAMINATED PLATES/SHELLS USING THE REFINED ZIGZAG THEORY
}

\author{
A. Eijo, E. Oñate and S. Oller \\ International Center for Numerical Methods in Engineering (CIMNE) \\ Universitat Politècnica de Catalunya (UPC) \\ Campus Norte UPC, 08034 Barcelona, Spain
}

\begin{abstract}
A new bilinear 4-noded quadrilateral element (called QLRZ) for the analysis of composite laminated and sandwich plates/shells based on the refined zigzag theory (RZT) proposed by Tessler et al. [1] is presented. The element has seven kinematic variables per node. Shear locking is avoided by introducing an assumed linear shear strain field. The performance of the element is studied in several examples where the reference solution is the 3D finite element analysis using 20-noded hexahedral elements. Finally, the capability for capturing delamination effects is analyzed.
\end{abstract}

\section{INTRODUCTION}

Classical plate thin theory, known as Kirchhoff theory [2], and the more advanced Reissner-Mindlin theory (RMT) [3, 4], also called First Order Shear Deformation Theory (FSDT), were the first simplified theories capable to precisely model a plate structure of homogeneous material. However, when applied to highly heterogeneous laminated composite plates it is known that both theories give poor predictions. The

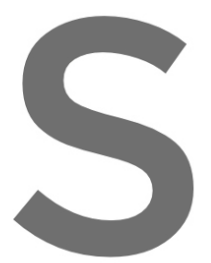
cause of this drawback is due to that these theories
distribution of the axial displacement, which is unable to
kinematics of a composite laminate
3D finite element analysis is the more appropriate tool
and shells of laminated composite material. However, for plies, 3D analysis becomes prohibitively expensive.

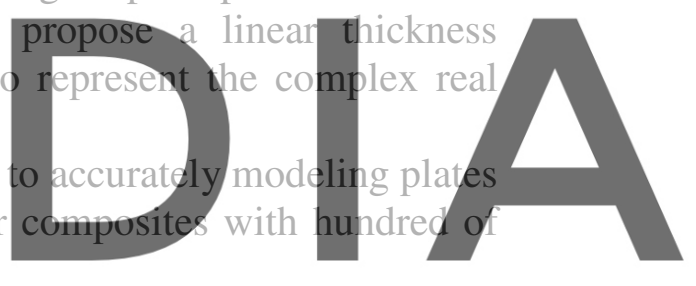

Improved FSDT models have been obtained by the so-called.Higher Order Shear

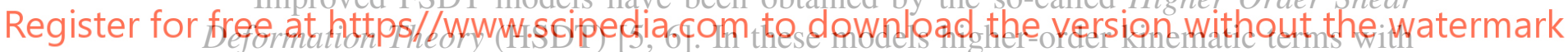

respect to the plate thickness are added to the expression for the axial displacement. However, these modeis are not effective for complex cases with localized loads or high transverse anisotropy.

More accurate models are given by Layer-Wise Theories (LWT) [5, 7] in which the thickness coordinate is divided into a number of analysis layers (that may be not coincident with the number of laminate layers) assuming separate displacement field expansions within each ply. LWT yields high quality predictions. However, the number of unknowns is proportional to the number of analysis layers, which largely increases the computational cost of the method.

An attractive alternative between the accuracy of LWT and the computational efficiency of FSDT and some HSDT are the ZigZag (ZZ) theories [5, 6, 8]. In ZZ theories the in-plane displacement is a superposition of a piecewise linear displacement function (the zigzag function) over a linear, quadratic or cubic displacement field along the thickness direction. It is important to note that the number of kinematics variables in $\mathrm{ZZ}$ theories is independent of the number of layers. Many of the $\mathrm{ZZ}$ formulations suffer from their inability to model correctly a clamped boundary condition, which makes it difficult to satisfy equilibrium of forces at a support. In addition many $\mathrm{ZZ}$ theories require $C^{1}$ continuity for the deflection field, which is a disadvantage versus simpler $C^{0}$ continuity plate theories, such as RMT. 
Tessler et al. [1,9] have recently developed a Refined ZigZag Theory (RZT) for beams and plates that adopt Timoshenko and RMT displacement fields as the baselines for beam and plate analysis, respectively. The key attributes of the RZT are, first, a linear piecewise zigzag function that vanishes at top and bottom surfaces of the beam and plate section. Second, it does not require full transverse shear stress continuity across the laminated plate depth. Third, $\mathrm{C}^{0}$ continuity is only required for the finite element method (FEM) approximation of the kinematic variables and finally, all boundary conditions can be effectively simulated [1,9-11].

Oñate et al. [10] have taken the RZT as the basis for developing a simple two-noded $\mathrm{C}^{0}$ beam element named LRZ. The accuracy of the LRZ beam element for analyzing composite laminated beams has been demonstrated for simple support and clamped beams under different loads. The possibility of the LRZ beam element for modeling delamination effects has also been tested $[10,12]$. More recently, anisoparametric two and three-noded $\mathrm{C}^{0}$ beam elements based in the RZT have been presented by Gherlone et al. [11].

In this work we present the formulation of an isoparametric four-noded $C^{0}$ quadrilateral plate element named QLRZ with seven kinematic variables per node based on the RZT [1]. Shear locking is avoided by using an assumed linear shear strain field. A deep study of this phenomenon is presented in annex I. The good performance of QLRZ is shown in three different studies: verification, convergence, and comparison. The verification section aims at evaluating the performance of this element when the material is homogenous, i.e., when the zigzag function vanishes. The influence of

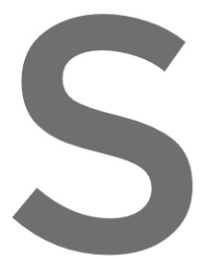
composite materia in the convergence of the QLRZ element the possibility of the laminated plate is dert
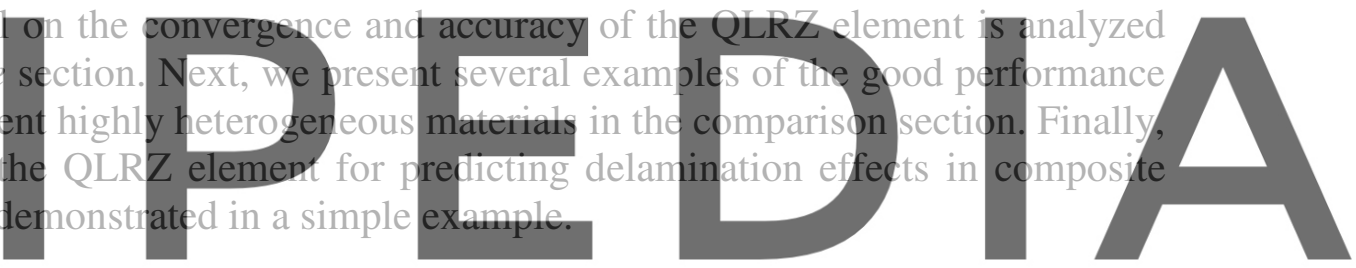

\section{GENERAL CONCEPTS OF ZIGZAG PLATE THEORY}

Register for free at https//www.scipedia.com to download the version without the watermark 2.1 ZIGZAG KINEMATICS

The kinematic field in zigzag plate theory is generally written as

$$
\begin{aligned}
& u^{k}(x, y, z)=u_{0}(x, y)-z \cdot \theta_{x}(x, y)+\bar{u}^{k}(x, y, z) \\
& v^{k}(x, y, z)=v_{0}(x, y)-z \cdot \theta_{y}(x, y)+\bar{v}^{k}(x, y, z) \\
& w(x, y)=w_{0}(x, y)
\end{aligned}
$$

where the axial displacement functions are

$$
\begin{aligned}
& \bar{u}^{k}=\phi_{x}^{k}(z) \cdot \psi_{x}(x, y) \quad ; \quad k=1, N \\
& \bar{v}^{k}=\phi_{y}^{k}(z) \cdot \psi_{y}(x, y)
\end{aligned}
$$

and superscript $k$ indicates quantities within the $k$ th layer with $z_{k} \leq z \leq z_{k+1}, z_{k}$ is the vertical coordinate of the $k$ th interface and $N$ is the number of layers. The uniform axial displacements along the coordinate directions $x$ and $y$ are $u_{0}$ and $v_{0}$, respectively; $\theta_{x}$ and $\theta_{y}$ represent the average bending rotation of the transverse normal about the positive $y$ and $x$ directions; and $w_{0}$ is the transverse deflection. $\phi_{i}^{k}(i=x, y)$ denotes a known piecewise linear zigzag function, and $\psi_{i}$ is a primary kinematic variable defining the amplitude of the zigzag function on the plate. Summarizing, the kinematic variables are 


$$
\mathbf{a}=\left[\begin{array}{lllllll}
u_{0} & v_{0} & w_{0} & \theta_{x} & \theta_{y} & \psi_{x} & \psi_{y}
\end{array}\right]^{T}
$$

The zigzag displacement field of Eq.(1a) is a superposition between the standard kinematics of the first order Reissner-Mindlin theory (RMT) and the linear piecewise zigzag functions (Eq.(1b)). Note that the zigzag displacement vanishes for homogeneous materials leading to the displacement field of the RMT.

The in-plane $\left(\varepsilon_{p}^{k}\right)$ and transverse shear $\left(\varepsilon_{t}^{k}\right)$ strains are defined as

$$
\boldsymbol{\varepsilon}^{k}=\left[\frac{\boldsymbol{\varepsilon}_{p}}{\boldsymbol{\varepsilon}_{t}}\right]^{k}=\left[\begin{array}{c}
\boldsymbol{\varepsilon}_{x} \\
\boldsymbol{\varepsilon}_{y} \\
\frac{\gamma_{x y}}{\gamma_{x z}} \\
\gamma_{y z}
\end{array}\right]^{k}\left[\begin{array}{c}
\frac{\partial u^{k}}{\partial x} \\
\frac{\partial v^{k}}{\partial y} \\
\frac{\partial u^{k}}{\partial y}+\frac{\partial v^{k}}{\partial x} \\
\frac{\partial u^{k}}{\partial z}+\frac{\partial w}{\partial x} \\
\frac{\partial v^{k}}{\partial z}+\frac{\partial w}{\partial y}
\end{array}\right]
$$
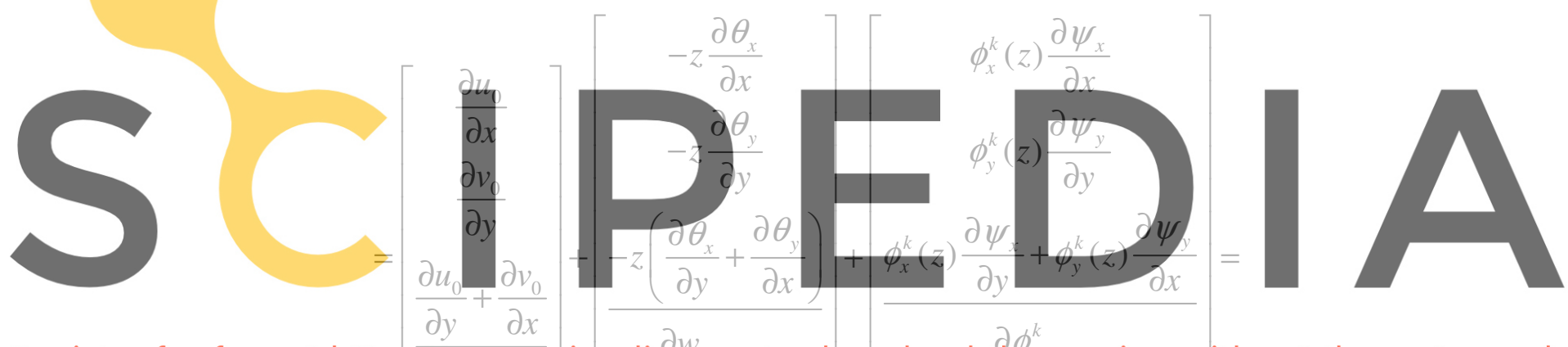

Register for free at https//WWW.scipedia.comoto download the

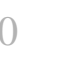

$$
\begin{gathered}
-\left[\begin{array}{c}
\frac{\partial w_{0}}{\partial y}-\theta_{y} \\
]
\end{array} \frac{\partial \phi_{y}^{k}}{\partial z} \psi_{y}\right. \\
=\left[\begin{array}{c}
\boldsymbol{\varepsilon}_{m} \\
\mathbf{0}
\end{array}\right]+\left[\begin{array}{c}
\boldsymbol{\varepsilon}_{b} \\
\boldsymbol{\varepsilon}_{s}
\end{array}\right]+\left[\begin{array}{c}
\boldsymbol{\varepsilon}_{m b \phi} \\
\boldsymbol{\varepsilon}_{s \phi}
\end{array}\right]^{k}=\left[\begin{array}{cc}
\mathbf{S}_{p} & \mathbf{0} \\
\mathbf{0} & \mathbf{S}_{t}
\end{array}\right]^{k} \cdot\left[\begin{array}{c}
\hat{\boldsymbol{\varepsilon}}_{p} \\
\hat{\boldsymbol{\varepsilon}}_{t}
\end{array}\right]
\end{gathered}
$$

where $\boldsymbol{\varepsilon}_{m}, \boldsymbol{\varepsilon}_{b}$ and $\boldsymbol{\varepsilon}_{s}$ are the strain vectors duo to membrane, bending and transverse shear effects of the RMT, respectively. The in-plane and transverse shear strains vectors emanating from the RZT are denoted by $\boldsymbol{\varepsilon}_{m b \phi}$ and $\boldsymbol{\varepsilon}_{s \phi}$, and $\hat{\boldsymbol{\varepsilon}}_{p}$ and $\hat{\boldsymbol{\varepsilon}}_{t}$ are the generalized in-plane and transverse shear strains vectors defined as

$$
\begin{aligned}
\hat{\boldsymbol{\varepsilon}}_{p}=\left[\begin{array}{c}
\hat{\boldsymbol{\varepsilon}}_{m} \\
\hat{\boldsymbol{\varepsilon}}_{b} \\
\hat{\boldsymbol{\varepsilon}}_{m b \phi}
\end{array}\right] \quad ; \quad \hat{\boldsymbol{\varepsilon}}_{t}=\left[\begin{array}{l}
\hat{\boldsymbol{\varepsilon}}_{s} \\
\hat{\boldsymbol{\varepsilon}}_{s \phi}
\end{array}\right] \\
\mathbf{S}_{p}^{k}=\left[\begin{array}{lll}
\mathbf{S}_{m} & \mathbf{S}_{b} & \mathbf{S}_{m b \phi}^{k}
\end{array}\right] \quad ; \quad \mathbf{S}_{t}^{k}=\left[\begin{array}{ll}
\mathbf{S}_{s} & \mathbf{S}_{s \phi}^{k}
\end{array}\right]
\end{aligned}
$$

where (.) denotes the generalized strain vectors given by 


$$
\begin{aligned}
& \hat{\boldsymbol{\varepsilon}}_{m}=\left[\begin{array}{lll}
\frac{\partial u_{0}}{\partial x} & \frac{\partial v_{0}}{\partial y} & \frac{\partial u_{0}}{\partial y}+\frac{\partial v_{0}}{\partial x}
\end{array}\right]^{T} \quad ; \quad \hat{\boldsymbol{\varepsilon}}_{s}=\left[\begin{array}{l}
\frac{\partial w_{0}}{\partial x}-\theta_{x} \\
\frac{\partial w_{0}}{\partial y}-\theta_{y}
\end{array}\right]=\left[\begin{array}{l}
\gamma_{x z} \\
\gamma_{y z}
\end{array}\right] \\
& \hat{\boldsymbol{\varepsilon}}_{b}=\left[\begin{array}{lll}
\frac{\partial \theta_{x}}{\partial x} & \frac{\partial \theta_{y}}{\partial y} & \frac{\partial \theta_{x}}{\partial y}+\frac{\partial \theta_{y}}{\partial x}
\end{array}\right]^{T} \quad ; \quad \hat{\boldsymbol{\varepsilon}}_{s \phi}=\left[\begin{array}{l}
\psi_{x} \\
\psi_{y}
\end{array}\right] \\
& \hat{\boldsymbol{\varepsilon}}_{m b \phi}=\left[\begin{array}{llll}
\frac{\partial \psi_{x}}{\partial x} & \frac{\partial \psi_{y}}{\partial y} & \frac{\partial \psi_{x}}{\partial y} & \frac{\partial \psi_{y}}{\partial x}
\end{array}\right]^{T} \\
& \mathbf{S}_{m}=\left[\begin{array}{lll}
1 & 0 & 0 \\
0 & 1 & 0 \\
0 & 0 & 1
\end{array}\right]=\mathbf{I}_{3} \quad ; \quad \mathbf{S}_{b}=-z \mathbf{I}_{3} \quad ; \quad \mathbf{S}_{s}=\left[\begin{array}{ll}
1 & 0 \\
0 & 1
\end{array}\right]=\mathbf{I}_{2} \\
& \mathbf{S}_{m b \phi}^{k}=\left[\begin{array}{cccc}
\phi_{x}^{k}(z) & 0 & 0 & 0 \\
0 & \phi_{y}^{k}(z) & 0 & 0 \\
0 & 0 & \phi_{x}^{k}(z) & \phi_{y}^{k}(z)
\end{array}\right] ; \quad \mathbf{S}_{s \phi}^{k}=\left[\begin{array}{cc}
\frac{\partial \phi_{x}^{k}}{\partial z} & 0 \\
0 & \frac{\partial \phi_{y}^{k}}{\partial z}
\end{array}\right]
\end{aligned}
$$

where $\gamma_{i z}(i=x, y)$ is the average transverse shear strain of RMT. Note that $\phi_{i}^{k}$ is

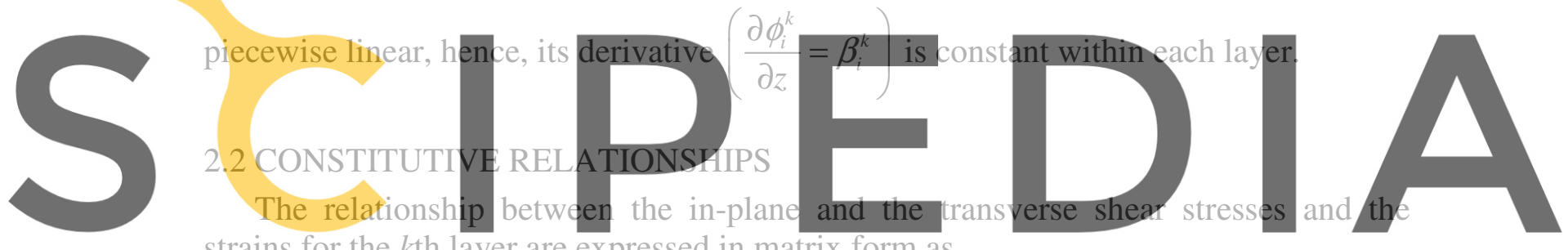
strains for the $k$ th layer are expressed in matrix form as

Register for free at https//www.scipedia. $\emptyset^{\prime k}$ to download the version without the watermark

$$
\boldsymbol{\sigma}^{k}=\left[\frac{\sigma_{p}}{\boldsymbol{\sigma}_{t}}\right]^{k}=\left[\begin{array}{l}
\sigma_{y} \\
\frac{\tau_{x y}}{\tau_{x z}} \\
\tau_{y z}
\end{array}\right]=\left[\begin{array}{cc}
D_{p} & 0 \\
\mathbf{0} & \mathbf{D}_{t}
\end{array}\right]^{k} \cdot\left[\begin{array}{l}
\varepsilon_{p} \\
\boldsymbol{\varepsilon}_{t}
\end{array}\right]^{k}=\mathbf{D}^{k} \boldsymbol{\varepsilon}^{k}
$$

with

$$
\begin{aligned}
\mathbf{D}_{p}^{k} & =\frac{1}{1-v_{x y} v_{y x}}\left[\begin{array}{ccc}
E_{x} & v_{x y} E_{x} & 0 \\
v_{y x} E_{x} & E_{y} & 0 \\
0 & 0 & \left(1-v_{x y} v_{y x}\right) G_{x y}
\end{array}\right] \\
\mathbf{D}_{t}^{k} & =\left[\begin{array}{cc}
G_{x z} & 0 \\
0 & G_{y z}
\end{array}\right]
\end{aligned}
$$

The resultant stress vectors are defined as Membrane forces 


$$
\begin{gathered}
\hat{\boldsymbol{\sigma}}_{m}=\left[\begin{array}{c}
N_{x} \\
N_{y} \\
N_{x y}
\end{array}\right]=\int_{z} \mathbf{S}_{m}{ }^{T} \boldsymbol{\sigma}_{p}^{k} d z \\
\hat{\boldsymbol{\sigma}}_{m}=\left(\int_{z} \mathbf{S}_{m}{ }^{T} \mathbf{D}_{p}^{k} \mathbf{S}_{m} d z\right) \hat{\boldsymbol{\varepsilon}}_{m}+\left(\int_{z} \mathbf{S}_{m}{ }^{T} \mathbf{D}_{p}^{k} \mathbf{S}_{b} d z\right) \hat{\boldsymbol{\varepsilon}}_{b}+\left(\int_{z} \mathbf{S}_{m}{ }^{T} \mathbf{D}_{p}^{k} \mathbf{S}_{m b \phi}^{k} d z\right) \hat{\boldsymbol{\varepsilon}}_{m b \phi} \\
\hat{\boldsymbol{\sigma}}_{m}=\hat{\mathbf{D}}_{m} \hat{\boldsymbol{\varepsilon}}_{m}+\hat{\mathbf{D}}_{m b} \hat{\boldsymbol{\varepsilon}}_{b}+\hat{\mathbf{D}}_{m m b \phi} \hat{\boldsymbol{\varepsilon}}_{m b \phi} \\
\hat{\mathbf{D}}_{m}=\int_{z} \mathbf{S}_{m}{ }^{T} \mathbf{D}_{p}^{k} \mathbf{S}_{m} d z \\
\hat{\mathbf{D}}_{m b}=\int_{z} \mathbf{S}_{m}{ }^{T} \mathbf{D}_{p}^{k} \mathbf{S}_{b} d z \\
\hat{\mathbf{D}}_{m m b \phi}=\int_{z} \mathbf{S}_{m}{ }^{T} \mathbf{D}_{p}^{k} \mathbf{S}_{m b \phi}^{k} d z
\end{gathered}
$$

Bending moments

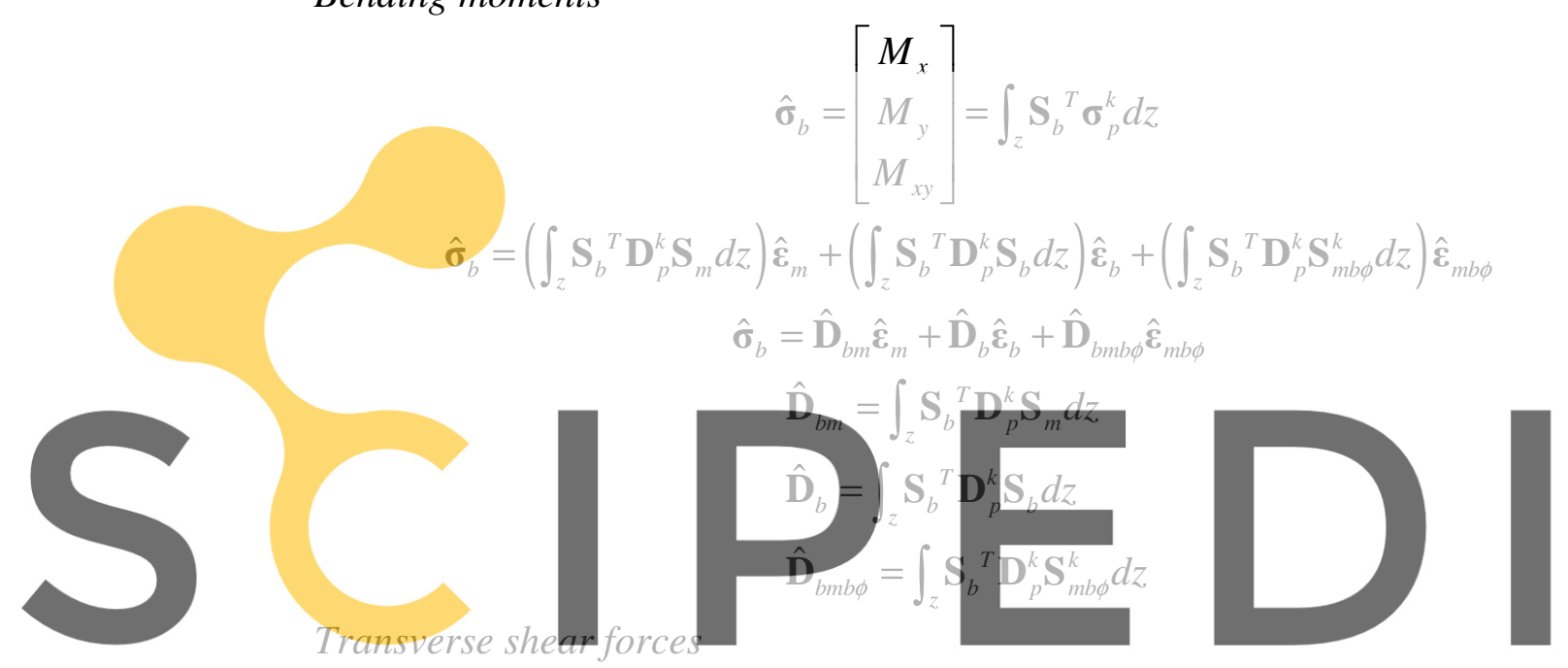

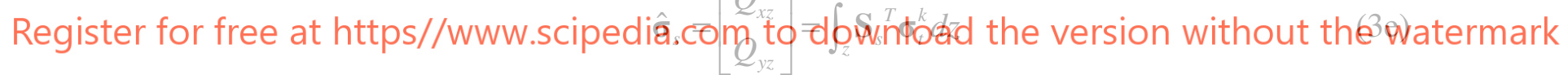

$$
\begin{gathered}
\hat{\mathbf{\sigma}}_{s}=\left(\int_{z} \mathbf{S}_{s}^{T} \mathbf{D}_{t}^{k} \mathbf{S}_{s} d z\right) \hat{\boldsymbol{\varepsilon}}_{s}+\left(\int_{z} \mathbf{S}_{s}^{T} \mathbf{D}_{t}^{k} \mathbf{S}_{s \phi}^{k} d z\right) \hat{\boldsymbol{\varepsilon}}_{s \phi} \\
\hat{\boldsymbol{\sigma}}_{s}=\hat{\mathbf{D}}_{s} \hat{\boldsymbol{\varepsilon}}_{s}+\hat{\mathbf{D}}_{s s \phi} \hat{\boldsymbol{\varepsilon}}_{s \phi} \\
\hat{\mathbf{D}}_{s}=\int_{z} \mathbf{S}_{s}{ }^{T} \mathbf{D}_{t}^{k} \mathbf{S}_{s} d z \\
\hat{\mathbf{D}}_{s s \phi}=\int_{z} \mathbf{S}_{s}^{T} \mathbf{D}_{t}^{k} \mathbf{S}_{s \phi}^{k} d z
\end{gathered}
$$

Next, we define the additional pseudo-bending moments and the pseudo-shear forces emanating from the RZT, which are conjugate to the new generalized strains $\frac{\partial \psi_{i}}{\partial j}(i, j=x, y)$ and the variable $\psi_{i}$, respectively.

The pseudo-bending moments are defined by

$$
\hat{\boldsymbol{\sigma}}_{m b \phi}=\left[\begin{array}{c}
M_{x \phi_{x}} \\
M_{y \phi_{y}} \\
M_{x y \phi_{x}} \\
M_{x y \phi_{y}}
\end{array}\right]=\int_{z} \mathbf{S}_{m b \phi}^{k}{ }^{T} \boldsymbol{\sigma}_{p}^{k} d z
$$




$$
\begin{aligned}
\hat{\boldsymbol{\sigma}}_{m b \phi}=\left(\int_{z} \mathbf{S}_{m b \phi}^{k}{ }^{T} \mathbf{D}_{p}^{k} \mathbf{S}_{m} d z\right) \hat{\boldsymbol{\varepsilon}}_{m}+\left(\int_{z} \mathbf{S}_{m b \phi}^{k}{ }^{T} \mathbf{D}_{p}^{k} \mathbf{S}_{b} d z\right) \hat{\boldsymbol{\varepsilon}}_{b}+\left(\int_{z} \mathbf{S}_{m b \phi}^{k}{ }^{T} \mathbf{D}_{p}^{k} \mathbf{S}_{m b \phi}^{k} d z\right) \hat{\boldsymbol{\varepsilon}}_{m b \phi} \\
\hat{\boldsymbol{\sigma}}_{m b \phi}=\hat{\mathbf{D}}_{m b \phi m} \hat{\boldsymbol{\varepsilon}}_{m}+\hat{\mathbf{D}}_{m b b \boldsymbol{b}} \hat{\boldsymbol{\varepsilon}}_{b}+\hat{\mathbf{D}}_{m b \phi} \hat{\boldsymbol{\varepsilon}}_{m b \phi} \\
\hat{\mathbf{D}}_{m b \phi m}=\int_{z} \mathbf{S}_{m b \phi}^{k} \mathbf{D}_{p}^{k} \mathbf{S}_{m} d z \\
\hat{\mathbf{D}}_{m b \phi b}=\int_{z} \mathbf{S}_{m b \phi}^{k} \mathbf{D}_{p}^{k} \mathbf{S}_{b} d z \\
\hat{\mathbf{D}}_{m b \phi}=\int_{z} \mathbf{S}_{m b \phi}^{k}{ }^{T} \mathbf{D}_{p}^{k} \mathbf{S}_{m b \phi}^{k} d z
\end{aligned}
$$

and the pseudo-shear forces by

$$
\begin{gathered}
\hat{\boldsymbol{\sigma}}_{s}=\left[\begin{array}{c}
Q_{x z \psi_{x}} \\
Q_{y z \psi_{y}}
\end{array}\right]=\int_{z} \mathbf{S}_{s \phi}^{k^{T}} \boldsymbol{\sigma}_{t}^{k} d z \\
\hat{\boldsymbol{\sigma}}_{s \phi}=\left(\int_{z} \mathbf{S}_{s \phi}^{k^{T}} \mathbf{D}_{t}^{k} \mathbf{S}_{s} d z\right) \hat{\boldsymbol{\varepsilon}}_{s}+\left(\int_{z} \mathbf{S}_{s \phi}^{k^{T}} \mathbf{D}_{t}^{k} \mathbf{S}_{s \phi}^{k} d z\right) \hat{\boldsymbol{\varepsilon}}_{s \phi} \\
\hat{\sigma}_{s \phi}=\hat{\mathbb{D}}_{s \phi s} \hat{\boldsymbol{\varepsilon}}_{s}+\hat{\mathbb{D}}_{s \phi} \hat{\boldsymbol{\varepsilon}}_{s \phi} \\
\hat{\mathbb{D}}_{s \phi s}=\int_{z} S_{s \phi}^{k^{T}} \mathbb{D}_{t}^{k} \mathrm{~S}_{s} d z \\
\hat{\mathbb{D}}_{s \phi}=\int_{z} \mathrm{~S}_{s \phi}^{k^{T}} \mathbb{D}_{t}^{k} \mathrm{~S}_{s \phi}^{k} d z
\end{gathered}
$$

The overall constitutive expression for the resultant stresses can be written in matrix form as
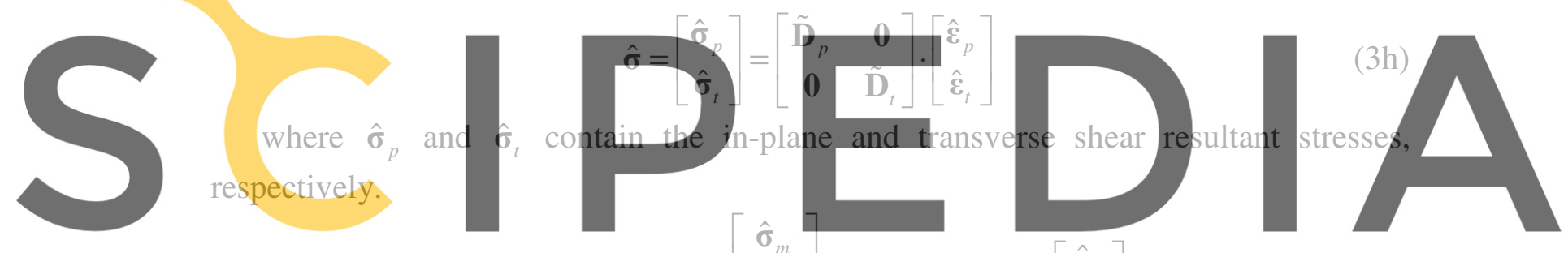

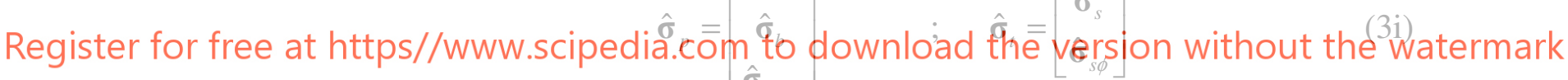

The in-piane and transverse shear generaiized constitutive matrices, $\tilde{\mathbf{D}}_{p}$ and $\tilde{\mathbf{D}}_{t}$ are given by

$$
\mathbf{D}_{p}=\left[\begin{array}{ccc}
\hat{\mathbf{D}}_{m} & \hat{\mathbf{D}}_{m b} & \hat{\mathbf{D}}_{m m b \phi} \\
\hat{\mathbf{D}}_{b m} & \hat{\mathbf{D}}_{b} & \hat{\mathbf{D}}_{b m b \phi} \\
\hat{\mathbf{D}}_{m b \phi m} & \hat{\mathbf{D}}_{m b \phi b} & \hat{\mathbf{D}}_{m b \phi}
\end{array}\right] \quad ; \quad \tilde{\mathbf{D}}_{t}=\left[\begin{array}{cc}
\hat{\mathbf{D}}_{s} & \hat{\mathbf{D}}_{s s \phi} \\
\hat{\mathbf{D}}_{s \phi s} & \hat{\mathbf{D}}_{s \phi}
\end{array}\right]
$$

\subsection{PRINCIPLE OF VIRTUAL WORK (PVW)}

The virtual work principle for a distributed load $q$ and point loads $\mathbf{f}_{i}$ can be stated as

$$
\iiint_{V} \boldsymbol{\delta} \boldsymbol{\varepsilon}^{k^{T}} \boldsymbol{\sigma}^{k} d V=\iint_{A} \boldsymbol{\delta} \mathbf{a}^{T} \mathbf{q} d A+\sum_{i=1}^{n p l} \boldsymbol{\delta} \mathbf{a}_{i}^{T} \mathbf{f}_{i}
$$

where the 1.h.s. of Eq.(4a) expresses the internal virtual work performed by the stresses and the r.h.s. is the external virtual work of the distributed and point loads. $V$ is the volume of the plate, $A$ is the area of application of the distributed load, and $n p l$ is the number of point loads. Substituting Eq.(2a) into Eq.(4a) gives 


$$
\begin{gathered}
\iiint_{V} \boldsymbol{\delta} \boldsymbol{\varepsilon}^{k T} \boldsymbol{\sigma}^{k} d V=\iiint_{V}\left(\boldsymbol{\delta} \hat{\boldsymbol{\varepsilon}}_{m}{ }^{T} \mathbf{S}_{m}{ }^{T}+\boldsymbol{\delta} \hat{\boldsymbol{\varepsilon}}_{b}{ }^{T} \mathbf{S}_{b}{ }^{T}+\boldsymbol{\delta} \hat{\boldsymbol{\varepsilon}}_{m b \phi}^{T} \mathbf{S}_{m b \phi}^{k}\right) \boldsymbol{\sigma}_{p}^{k} d V+ \\
\left.+\iiint_{V}\left(\boldsymbol{\delta} \hat{\boldsymbol{\varepsilon}}_{s}^{T} \mathbf{S}_{s}^{T}+\boldsymbol{\delta} \hat{\boldsymbol{s}}_{s \phi}{ }^{T} \mathbf{S}_{s \phi}^{k}\right)\right) \boldsymbol{\sigma}_{t}^{k} d V \\
=\iiint_{V}\left(\boldsymbol{\delta} \hat{\boldsymbol{\varepsilon}}_{m}{ }^{T} \mathbf{S}_{m}{ }_{m}^{T} \boldsymbol{\sigma}_{p}^{k}+\boldsymbol{\delta} \hat{\boldsymbol{\varepsilon}}_{b}{ }^{T} \mathbf{S}_{b}^{T}{ }^{T} \boldsymbol{\sigma}_{p}^{k}+\boldsymbol{\delta} \hat{\boldsymbol{\varepsilon}}_{m b \phi}^{T} \mathbf{S}_{m b \phi}^{k}{ }^{T} \boldsymbol{\sigma}_{p}^{k}\right) d V+ \\
+\iiint_{V}\left(\boldsymbol{\delta} \hat{\boldsymbol{\varepsilon}}_{s}{ }^{T} \mathbf{S}_{s}^{T} \boldsymbol{\sigma}_{t}^{k}+\boldsymbol{\delta} \hat{\boldsymbol{\varepsilon}}_{s \phi}{ }^{T} \mathbf{S}_{s \phi}^{k}{ }^{T} \boldsymbol{\sigma}_{t}^{k}\right) d V
\end{gathered}
$$

Using Eqs. (3c), (3d), (3e), (3f), (3g) yields

$$
\iiint_{V} \boldsymbol{\delta} \boldsymbol{\varepsilon}^{k T} \boldsymbol{\sigma}^{k} d V=\iint_{A}\left(\boldsymbol{\delta} \hat{\boldsymbol{\varepsilon}}_{m}^{T} \hat{\boldsymbol{\sigma}}_{m}+\boldsymbol{\delta} \hat{\boldsymbol{\varepsilon}}_{b}^{T} \hat{\boldsymbol{\sigma}}_{b}+\boldsymbol{\delta} \hat{\boldsymbol{\varepsilon}}_{m b \phi}{ }^{T} \hat{\boldsymbol{\sigma}}_{m b \phi}\right) d A+\iint_{A}\left(\boldsymbol{\delta} \hat{\boldsymbol{\varepsilon}}_{s}^{T} \hat{\boldsymbol{\sigma}}_{s}+\boldsymbol{\delta} \hat{\boldsymbol{\varepsilon}}_{s \phi}^{T} \hat{\boldsymbol{\sigma}}_{s \phi}\right) d A
$$

The virtual work can be therefore written as

$$
\iint_{A}\left(\delta \hat{\varepsilon}_{p}^{T} \hat{\boldsymbol{\sigma}}_{p}+\delta \hat{\varepsilon}_{t}^{T} \hat{\boldsymbol{\sigma}}_{t}\right)=\iint_{A} \delta \mathbf{a}^{T} \mathbf{q} d A+\sum_{i=1}^{n p l} \delta \mathbf{a}_{i}^{T} \mathbf{f}_{i}
$$

The integrands in Eq.(4b) contain kinematic variables derivatives up to first order only, which allows us to use $\mathrm{C}^{0}$ continuous elements.

\section{DERIVATION OF THE ZIGZAG FUNCTION}

The zigzag function is defined within each layer by
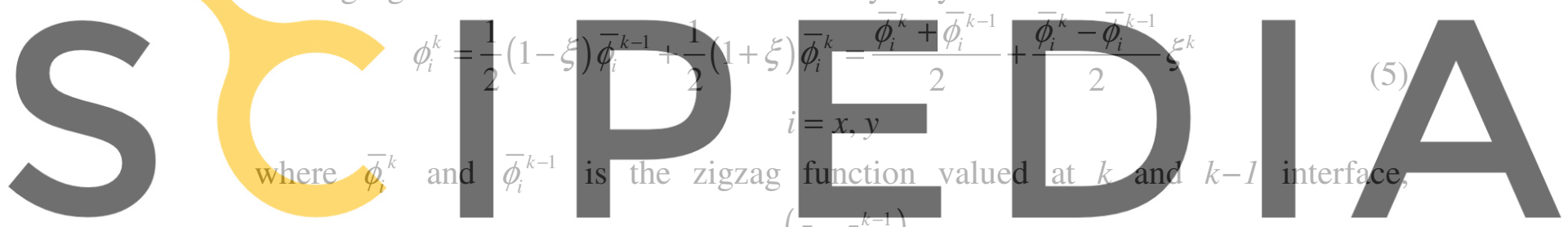

respectively with $\bar{\phi}_{i}^{0}=\bar{\phi}_{i}^{N}=0$ and $\xi^{k}=2 \frac{\left(z-z^{k-1}\right)}{c^{k}}-1$.

Register for free at https//www.scipedia.com to db ${ }^{k}$ wnload the version without the watermark

Figure (1) shows the zigzag function $\phi_{x}^{k}$, the zigzag displacements $\bar{u}^{k}$, and the axial

displacements $u^{k}$, for the $x$ direction. A similar distribution is found for the zigzag function $\phi_{y}^{k}$.

a)

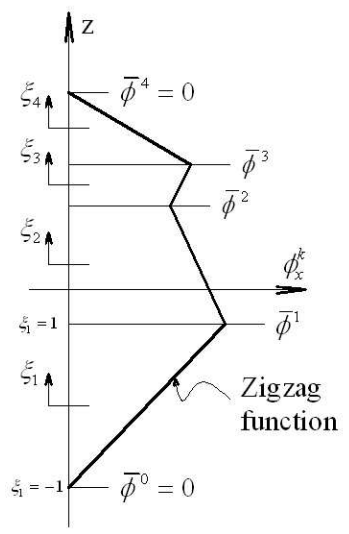

b)

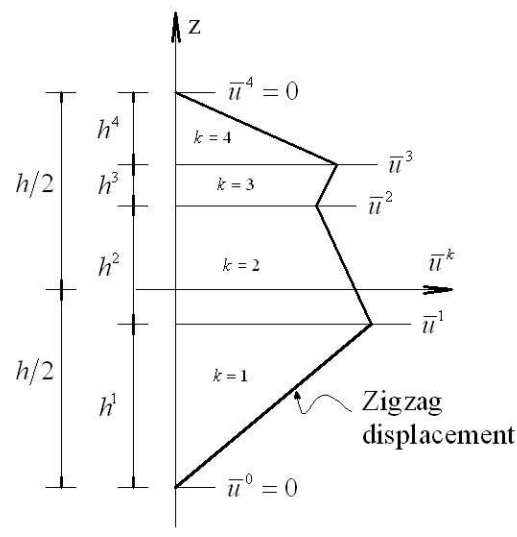

c)

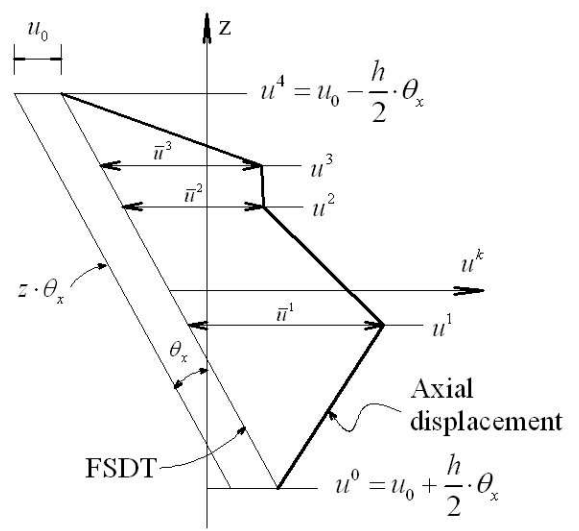

Figure 1 - Thickness distribution of the zigzag function $\phi_{x}^{k}$ a), zigzag displacement $\bar{u}^{k}$ b), and axial displacement $u^{k} \mathrm{c}$ ) in the RZT. 
The slope of the zigzag function (Eq.(5)) gives a constant value for each layer defined as

$$
\beta_{i}^{k}=\frac{\partial \phi_{i}^{k}}{\partial z}=\frac{\left(\bar{\phi}_{i}^{k}-\bar{\phi}_{i}^{k-1}\right)}{h^{k}}
$$

Because the zigzag function vanishes on the top and bottom surfaces, the throughthe-thickness integrals of the slope functions $\beta_{i}^{k}$ is equal to zero, i.e.

$$
\int_{z} \beta_{i}^{k} d z=0
$$

It is convenient to define a new different function $\eta_{i}$ as

$$
\eta_{i}=\gamma_{i z}-\psi_{i}
$$

which leads to the following expression for the $k$ th layer transverse shear strains and stresses as

$$
\begin{gathered}
\gamma_{i z}^{k}=\left(1+\beta_{i}^{k}\right) \gamma_{i z}-\beta_{i}^{k} \eta_{i} \\
\tau_{i z}^{k}=G_{i z}^{k}\left(1+\beta_{i}^{k}\right) \gamma_{i z}-G_{i z}^{k} \beta_{i}^{k} \eta_{i}
\end{gathered}
$$

The average shear strains over the plate thickness are obtained by integrating the transverse shear strains $\gamma_{i z}^{k}$ (Eq.(8a)) over the thickness and using Eq.(6b). This gives

$$
\gamma_{i z}=\frac{1}{h} \int_{z} \gamma_{i z}^{k} d z
$$

The interfacial continuity of the first term, associated with the average shear strain $\gamma_{i z}$, is enforced in the transverse shear stress distribution (Eq.(8b)), i.e.

$$
G_{i z}^{k}\left(1+\beta_{i}^{k}\right)=G_{i z}^{k+1}\left(1+\beta_{i}^{k+1}\right)
$$

which leads to a constant shear modulus across the plate thickness defined by

$$
G_{i z}=G_{i z}^{k}\left(1+\beta_{i}^{k}\right)
$$

Then, from Eq. (10b)

$$
\beta_{i}^{k}=\frac{G_{i z}}{G_{i z}^{k}}-1
$$

the explicit form of $G_{i z}$ is obtained by substituting $\beta_{i}^{k}$ in the integral of Eq.(6b), i.e.

$$
G_{i z}=h\left[\sum_{k=1}^{N} \frac{h^{k}}{G_{i z}^{k}}\right]^{-1}
$$

Finally, the zigzag function is obtained by replacing Eq.(6a) into Eq.(5), this gives

$$
\phi_{i}^{k}=\bar{\phi}_{i}^{k-1}+\frac{h^{k} \beta_{i}^{k}}{2}\left(\zeta^{k}+1\right)
$$

with $\beta_{i}^{k}$ defined by Eq.(11). 


\section{QUADRILATERAL LINEAR REFINED ZIGZAG PLATE ELEMENT (QLRZ)}

The QLRZ element (figure AI.2) is a Lagrangian isoparametric 4-noded finite element derived from the refined zigzag theory described above.

\subsection{DISCRETIZATION OF THE DISPLACEMENT FIELD}

The middle surface of a plate is discretized into 4-node 2D isoparametric finite elements of quadrilateral shape. The kinematic variables (Eq.(1c)) can be interpolated within each element as

$$
\mathbf{a}=\left[\begin{array}{c}
u_{0} \\
v_{0} \\
w_{0} \\
\theta_{x} \\
\theta_{y} \\
\psi_{x} \\
\psi_{y}
\end{array}\right]=\sum_{i=1}^{4} \mathbf{N}_{i} \mathbf{a}_{i}^{(e)}=\left[\begin{array}{llll}
\mathbf{N}_{1} & \mathbf{N}_{2} & \mathbf{N}_{3} & \mathbf{N}_{4}
\end{array}\right] \cdot\left[\begin{array}{c}
\mathbf{a}_{1}^{(e)} \\
\mathbf{a}_{2}^{(e)} \\
\mathbf{a}_{3}^{(e)} \\
\mathbf{a}_{4}^{(e)}
\end{array}\right]=\mathbf{N a}^{(e)}
$$

where

$$
\mathbf{N}_{i}=N_{i} \mathbf{I}_{7} \quad ; \quad \mathbf{a}_{i}^{(e)}=\left[\begin{array}{lllllll}
u_{0} & v_{0} & w_{0} & \theta_{x} & \theta_{y} & \psi_{x} & \psi_{y}
\end{array}\right]_{i}^{T}
$$

being $N_{i}(\xi, \eta)$ (Eq.(21)) the $\mathrm{C}^{0}$ continuous shape function of node $i$ th and $\mathbf{I}_{7}$ is the $7 \times 7$ unit matrix.

\subsection{GENERALIZED STRAIN FIELD}

The generalized in-plane strains are obtained in terms of the nodal kinematic variables by substituting Eq.(14) into the generalized in-plane shear strains $\hat{\varepsilon}_{p}$ (Eq.(2b)), 


$$
\hat{\boldsymbol{\varepsilon}}_{p}=\left[\begin{array}{c}
\hat{\boldsymbol{\varepsilon}}_{m} \\
\hat{\boldsymbol{\varepsilon}}_{b} \\
\hat{\boldsymbol{\varepsilon}}_{m b \phi}
\end{array}\right]=\left[\begin{array}{c}
\frac{\partial u_{0}}{\partial x} \\
\frac{\partial v_{0}}{\partial y} \\
\frac{\partial u_{0}}{\partial y}+\frac{\partial v_{0}}{\partial x} \\
\frac{\partial \theta_{x}}{\partial x} \\
\frac{\partial \theta_{y}}{\partial y} \\
\frac{\partial \theta_{x}}{\partial y}+\frac{\partial \theta_{y}}{\partial x} \\
\frac{\partial \psi_{x}}{\partial x} \\
\frac{\partial \psi_{y}}{\partial y} \\
\frac{\partial \psi_{x}}{\partial y} \\
\frac{\partial \psi_{y}}{\partial y}
\end{array}\right]=\sum_{i=1}^{4}\left[\begin{array}{c}
\frac{\partial N_{i}}{\partial x} u_{0} \\
\frac{\partial N_{i}}{\partial y} v_{0} \\
\frac{\partial N_{i}}{\partial x} \theta_{x} \\
\frac{\partial N_{i}}{\partial y} \theta_{y}+\frac{\partial N_{i}}{\partial x} \theta_{y} \\
\frac{\partial N_{i}}{\partial x} \psi_{x} \\
\frac{\partial N_{i}}{\partial y} \psi_{y} \\
\frac{\partial N_{i}}{\partial y} \psi_{x} \\
\frac{\partial N_{i}}{\partial x} \psi_{y}
\end{array}\right]_{i=1}^{4} \mathbf{B}_{p i} \mathbf{a}_{i}^{(e)}=\mathbf{B}_{p} \mathbf{a}^{(e)}
$$

where $\mathbf{B}_{p}$ and $\mathbf{B}_{p i}$ are the in-plane generalized strain matrices for the element and the $i$ th node, respectively. The matrix $\mathbf{B}_{p i}$ can be splitted into membrane $(m)$, bending $(b)$ and zigzag $(m b \phi)$ contributions. This leads to

$$
\mathbf{B}_{p i}=\left[\begin{array}{c}
\mathbf{B}_{m} \\
\mathbf{B}_{b} \\
\mathbf{B}_{m b \phi}
\end{array}\right]_{i}
$$

with

$$
\begin{aligned}
& \mathbf{B}_{m i}=\left[\begin{array}{ccccccc}
\frac{\partial N_{i}}{\partial x} & 0 & 0 & 0 & 0 & 0 & 0 \\
0 & \frac{\partial N_{i}}{\partial y} & 0 & 0 & 0 & 0 & 0 \\
\frac{\partial N_{i}}{\partial y} \frac{\partial N_{i}}{\partial x} & 0 & 0 & 0 & 0 & 0
\end{array}\right] \mathbf{B}_{b i}=\left[\begin{array}{ccccccc}
0 & 0 & 0 & \frac{\partial N_{i}}{\partial x} & 0 & 0 & 0 \\
0 & 0 & 0 & 0 & \frac{\partial N_{i}}{\partial y} & 0 & 0 \\
0 & 0 & 0 & \frac{\partial N_{i}}{\partial y} \frac{\partial N_{i}}{\partial x} & 0 & 0
\end{array}\right] \\
& \mathbf{B}_{m b \phi i}=\left[\begin{array}{ccccccc}
0 & 0 & 0 & 0 & 0 & \frac{\partial N_{i}}{\partial x} & 0 \\
0 & 0 & 0 & 0 & 0 & 0 & \frac{\partial N_{i}}{\partial y} \\
0 & 0 & 0 & 0 & 0 & \frac{\partial N_{i}}{\partial y} & 0 \\
0 & 0 & 0 & 0 & 0 & 0 & \frac{\partial N_{i}}{\partial x}
\end{array}\right]
\end{aligned}
$$

Replacing Eq.(14) into (Eq.(2b)) the generalized transverse strains are obtained as 


$$
\hat{\boldsymbol{\varepsilon}}_{t}=\left[\begin{array}{c}
\hat{\boldsymbol{\varepsilon}}_{s} \\
\hat{\boldsymbol{\varepsilon}}_{s \phi}
\end{array}\right]=\left[\begin{array}{c}
\frac{\partial w_{0}}{\partial x}-\theta_{x} \\
\frac{\partial w_{0}}{\partial y}-\theta_{y} \\
\psi_{x} \\
\psi_{y}
\end{array}\right]=\sum_{i=1}^{4}\left[\begin{array}{c}
\frac{\partial N_{i}}{\partial x} w_{0}-N_{i} \theta_{x} \\
\frac{\partial N_{i}}{\partial y} w_{0}-N_{i} \theta_{y} \\
N_{i} \psi_{x} \\
N_{i} \psi_{y}
\end{array}\right]_{i} \sum_{i=1}^{4} \mathbf{B}_{t i} \mathbf{a}_{i}^{(e)}=\mathbf{B}_{t} \mathbf{a}^{(e)}
$$

where $\mathbf{B}_{t}$ and $\mathbf{B}_{t i}$ are the transverse generalized strain matrices for the $e$ element and the $i$ th node, respectively. Matrix $\mathbf{B}_{t i}$ can be split into shear $(s)$ and zigzag $(s \phi)$ contributions as

$$
\mathbf{B}_{t i}=\left[\begin{array}{c}
\mathbf{B}_{s} \\
\mathbf{B}_{s \phi}
\end{array}\right]_{i}
$$

where

$$
\begin{aligned}
\mathbf{B}_{s i} & =\left[\begin{array}{ccccccc}
0 & 0 & \frac{\partial N_{i}}{\partial x} & -N_{i} & 0 & 0 & 0 \\
0 & 0 & \frac{\partial N_{i}}{\partial y} & 0 & -N_{i} & 0 & 0
\end{array}\right] \\
\mathbf{B}_{s \phi i} & =\left[\begin{array}{lllllcc}
0 & 0 & 0 & 0 & 0 & N_{i} & 0 \\
0 & 0 & 0 & 0 & 0 & 0 & N_{i}
\end{array}\right]
\end{aligned}
$$

\subsection{ELEMENT STIFFNESS MATRIX}

The equilibrium equations relating nodal forces and displacements are obtained by substituting the discretized equations (15a) and (16a) into the virtual work principle

$$
\iint_{A}\left(\boldsymbol{\delta} \hat{\boldsymbol{\varepsilon}}_{p}^{T} \hat{\boldsymbol{\sigma}}_{p}+\boldsymbol{\delta} \hat{\boldsymbol{\varepsilon}}_{t}^{T} \hat{\boldsymbol{\sigma}}_{t}\right)=\iint_{A} \boldsymbol{\delta} \mathbf{a}^{T} \mathbf{q} d A+\sum_{i=1}^{n p l} \boldsymbol{\delta} \mathbf{a}_{i}^{T} \mathbf{f}_{i}
$$

Substituting Eq.(3h) into the 1.h.s of Eq.(17a) gives

Considering that

$$
\iint_{A}\left(\boldsymbol{\delta} \hat{\boldsymbol{\varepsilon}}_{p}^{T} \hat{\boldsymbol{\sigma}}_{p}+\boldsymbol{\delta} \hat{\boldsymbol{\varepsilon}}_{t}^{T} \hat{\boldsymbol{\sigma}}_{t}\right)=\iint_{A}\left(\boldsymbol{\delta} \hat{\boldsymbol{\varepsilon}}_{p}^{T} \tilde{\mathbf{D}}_{p} \hat{\boldsymbol{\varepsilon}}_{p}+\boldsymbol{\delta} \hat{\boldsymbol{\varepsilon}}_{t}^{T} \tilde{\mathbf{D}}_{t} \hat{\boldsymbol{\varepsilon}}_{t} d A\right)
$$

$$
\begin{aligned}
\boldsymbol{\delta} \hat{\boldsymbol{\varepsilon}}_{p}^{T} & =\sum_{i=1}^{4} \boldsymbol{\delta} \mathbf{a}_{i}^{(e) T} \mathbf{B}_{p i}{ }^{T}=\boldsymbol{\delta} \mathbf{a}^{(\mathrm{e}) T} \mathbf{B}_{p}{ }^{T} \\
\boldsymbol{\delta} \hat{\boldsymbol{\varepsilon}}_{t}^{T} & =\sum_{i=1}^{4} \boldsymbol{\delta} \mathbf{a}_{i}^{(e) T} \mathbf{B}_{t i}{ }^{T}=\boldsymbol{\delta} \mathbf{a}^{(\mathrm{e}) T} \mathbf{B}_{t}{ }^{T}
\end{aligned}
$$

and substituting Eqs.(15a), (16a), (17c) into Eq.(17b) yields

$$
\begin{aligned}
\iint_{A}\left(\boldsymbol{\delta} \hat{\boldsymbol{\varepsilon}}_{p}^{T} \tilde{\mathbf{D}}_{p} \hat{\boldsymbol{\varepsilon}}_{p}+\boldsymbol{\delta} \hat{\boldsymbol{\varepsilon}}_{t}^{T} \tilde{\mathbf{D}}_{t} \hat{\boldsymbol{\varepsilon}}_{t}\right) d A & =\iint_{A}\left(\boldsymbol{\delta} \mathbf{a}^{(\mathrm{e}) T} \mathbf{B}_{p}{ }^{T} \tilde{\mathbf{D}}_{p} \mathbf{B}_{p} \mathbf{a}^{(e)}\right) d A \\
& +\iint_{A}\left(\boldsymbol{\delta} \mathbf{a}^{(\mathrm{e}) T} \mathbf{B}_{t}{ }^{T} \tilde{\mathbf{D}}_{t} \mathbf{B}_{t} \mathbf{a}^{(e)}\right) d A= \\
= & \boldsymbol{\delta} \mathbf{a}^{(\mathrm{e}) T}\left[\iint_{A}\left(\mathbf{B}_{p}{ }^{T} \tilde{\mathbf{D}}_{p} \mathbf{B}_{p}+\mathbf{B}_{t}{ }^{T} \tilde{\mathbf{D}}_{t} \mathbf{B}_{t}\right) d A\right] \mathbf{a}^{(e)} \\
= & \boldsymbol{\delta} \mathbf{a}^{(\mathrm{e}) T} \mathbf{K}^{(e)} \mathbf{a}^{(e)}
\end{aligned}
$$


Finally Eq.(17a) is reduced to

$$
\mathbf{K}^{(e)} \mathbf{a}^{(e)}-\iint_{A} \mathbf{q} d A-\sum_{i=1}^{n p l} \mathbf{f}_{i}=0
$$

where $\mathbf{K}^{(e)}$ is the sought element stiffness matrix. This matrix can be expressed as

$$
\mathbf{K}^{(e)}=\mathbf{K}_{p}^{(e)}+\mathbf{K}_{t}^{(e)}
$$

being $\mathbf{K}_{p}^{(e)}$ and $\mathbf{K}_{t}^{(e)}$ the in-plane and transverse stiffness matrices, respectively. These are given by

$$
\begin{aligned}
\mathbf{K}_{p}^{(e)} & =\iint_{A} \mathbf{B}_{p}{ }^{T} \tilde{\mathbf{D}}_{p} \mathbf{B}_{p} d A \\
\mathbf{K}_{t}^{(e)} & =\iint_{A} \mathbf{B}_{t}{ }^{T} \tilde{\mathbf{D}}_{t} \mathbf{B}_{t} d A
\end{aligned}
$$

To facilitate subsequent shear locking studies, matrix $\mathbf{K}_{t}^{(e)}$ is split as follows

$$
\mathbf{K}_{t}^{(e)}=\mathbf{K}_{s}^{(e)}+\mathbf{K}_{s \phi}^{(e)}+\mathbf{K}_{s s \phi}^{(e)}+\left[\mathbf{K}_{s s \phi}^{(e)}\right]^{T}
$$

with

$$
\begin{aligned}
& \mathbf{K}_{s}^{(e)}=\iint_{A} \mathbf{B}_{s}{ }^{T} \hat{\mathbf{D}}_{s} \mathbf{B}_{s} d A \\
& \mathbf{K}_{s \phi}^{(e)}=\iint_{A} \mathbf{B}_{s \phi}{ }^{T} \hat{\mathbf{D}}_{s \phi} \mathbf{B}_{s \phi} d A \\
& \mathbf{K}_{s s \phi}^{(e)}=\iint_{A} \mathbf{B}_{s}{ }^{T} \hat{\mathbf{D}}_{s s \phi} \mathbf{B}_{s \phi} d A
\end{aligned}
$$

\subsection{BOUNDARY CONDITIONS}

The boundary conditions are:

A. Clamped side:

$$
\begin{gathered}
w=0 \\
u=\theta_{x}=\psi_{x}=0 \\
v=\theta_{y}=\psi_{y}=0
\end{gathered}
$$

B. Simply supported side:

- Hard support: $\quad w=u_{s}=\theta_{s}=\psi_{s}=0$

- Soft support: $\quad w=0$

where "s" is the direction of the side.

C. Symmetry axis:

$$
u_{n}=\theta_{n}=\psi_{n}=0
$$

where " $n$ " is the orthogonal direction to the symmetry axis.

\subsection{SHEAR LOCKING}

The original form of the QLRZ element suffers shear locking for slender composite laminated plates. In order to remove this defect two different alternatives are analyzed in Annex I: 1) by using a reduced integration of the transverse stiffness matrix $\mathbf{K}_{t}^{(e)}$ (Eq.(20a), and 2) by using an assumed transverse shear strain field [13]. The study showed that the assumed transverse shear strain technique is a more consistent alternative for avoiding the shear locking problem.

Matrices $\mathbf{B}_{m i}, \mathbf{B}_{b i}, \mathbf{B}_{m b \phi i}$ from Eq.(15c) and $\mathbf{B}_{s \phi i}$ (Eq.(16c)) are computed using bilinear shape functions (Eq.(21)) while matrix $\mathbf{B}_{s}$ (Eqs. (16b) and (AI.16.b)) is replaced by the substitute transverse shear strain matrix $\overline{\mathbf{B}}_{s}$ of Eq.(AI.18). 
The bi-linear shape functions $N_{i}$ are

$$
N_{i}=\frac{1}{4}\left(1+\xi \xi_{i}\right)\left(1+\eta \eta_{i}\right)
$$

Table 1 - Values of $\xi_{\mathrm{i}}$ and $\eta_{\mathrm{i}}$ for each node.

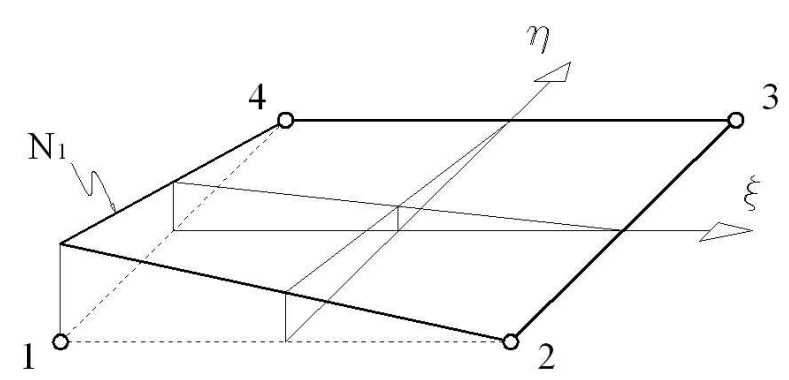

$$
\begin{aligned}
& \mathrm{N}_{1}=0.25(1-\xi)(1-\eta) \\
& \mathrm{N}_{2}=0.25(1+\xi)(1-\eta) \\
& \mathrm{N}_{3}=0.25(1+\xi)(1+\eta) \\
& \mathrm{N}_{4}=0.25(1-\xi)(1+\eta)
\end{aligned}
$$

Figure 2 - Bilinear shape functions.

The stiffness matrices $\mathbf{K}_{s}^{(e)}$ and $\mathbf{K}_{s s \phi}^{(e)}$ of $\mathbf{K}_{t}^{(e)}$ (Eq.(20a)) are computed as

$$
\begin{aligned}
& \mathbf{K}_{s}^{(e)}=\iint_{A} \overline{\mathbf{B}}_{s}^{T} \hat{\mathbf{D}}_{s} \overline{\mathbf{B}}_{s} d A \\
& \mathbf{K}_{s s \phi}^{(e)}=\iint_{A} \overline{\mathbf{B}}_{s}^{T} \hat{\mathbf{D}}_{s s \phi} \mathbf{B}_{s \phi} d A
\end{aligned}
$$

\section{6 “A POSTERIORI” COMPUTATION OF TRANSVERSE SHEAR STRESSES}

While in-plane stresses $\left(\sigma_{x}, \sigma_{y}\right.$ and $\left.\tau_{x y}\right)$ are well predicted by equation (3a), the transverse shear stresses $\left(\tau_{x z}\right.$ and $\left.\tau_{y z}\right)$ are not. The reason is that the constitutive Eq. (3a) yields a constant value into each layer, leading to a discontinuous thickness distribution of $\tau_{x z}$ and $\tau_{y z}$. An useful alternative is to compute $\tau_{x z}$ and $\tau_{y z}$ a posteriori from the in-plane stresses using the equilibrium equations,

$$
\begin{aligned}
& \frac{\partial \sigma_{x}}{\partial x}+\frac{\partial \tau_{x y}}{\partial y}+\frac{\partial \tau_{x z}}{\partial z}=0 \\
& \frac{\partial \tau_{x y}}{\partial x}+\frac{\partial \sigma_{y}}{\partial y}+\frac{\partial \tau_{y z}}{\partial z}=0
\end{aligned}
$$

from which, the transverse shear stresses at a point "P" across the thickness coordinates $z$ are computed by

$$
\begin{aligned}
& \left.\tau_{x z}(z)\right|_{P}=-\left.\int_{h / 2}^{z} \frac{\partial \sigma_{x}}{\partial x}\right|_{P} d z-\left.\int_{h / 2}^{z} \frac{\partial \tau_{x y}}{\partial y}\right|_{P} d z \\
& \left.\tau_{y z}(z)\right|_{P}=-\left.\int_{h / 2}^{z} \frac{\partial \sigma_{y}}{\partial y}\right|_{P} d z-\left.\int_{h / 2}^{z} \frac{\partial \tau_{x y}}{\partial x}\right|_{P} d z
\end{aligned}
$$


The in-plane stresses at point "P" in the QLRZ element are approximated by

$$
\begin{aligned}
& \left.\sigma_{x}(z)\right|_{P}=\left.\sum_{i=1}^{4} N_{i}\right|_{P} \cdot \sigma_{x}^{i}(z) \\
& \left.\sigma_{y}(z)\right|_{P}=\left.\sum_{i=1}^{4} N_{i}\right|_{P} \cdot \sigma_{y}^{i}(z) \\
& \left.\tau_{x y}(z)\right|_{P}=\left.\sum_{i=1}^{4} N_{i}\right|_{P} \cdot \tau_{x y}^{i}(z)
\end{aligned}
$$

where $N_{i}$ is the shape function (Eq.(21)) and $i$ denotes the $i$ th node. Finally, the transverse shear stresses are obtained by replacing Eq.(23c) into $\operatorname{Eq}(23 b)$,

$$
\begin{aligned}
& \left.\tau_{x z}(z)\right|_{P}=-\int_{h / 2}^{z}\left(\left.\sum_{i=1}^{4} \frac{\partial N_{i}}{\partial x}\right|_{P} \cdot \sigma_{x}^{i}(z)\right)-\int_{h / 2}^{z}\left(\left.\sum_{i=1}^{4} \frac{\partial N_{i}}{\partial y}\right|_{P} \cdot \tau_{x y}^{i}(z)\right) \\
& \left.\tau_{y z}(z)\right|_{P}=-\int_{h / 2}^{z}\left(\left.\sum_{i=1}^{4} \frac{\partial N_{i}}{\partial y}\right|_{P} \cdot \sigma_{y}^{i}(z)\right)-\int_{h / 2}^{z}\left(\left.\sum_{i=1}^{4} \frac{\partial N_{i}}{\partial x}\right|_{P} \cdot \tau_{x y}^{i}(z)\right)
\end{aligned}
$$

\section{VERIFICATION STUDIES}

The accuracy of the $Q L R Z$ element for isotropic homogeneous material is studied in this section. The aim is to evaluate the behavior of the QLRZ element when $\phi_{i}$ $(i=x, y)$ vanishes which leads to $\psi_{i}=0$ and the kinematics of Eq.(1a) coincide with that of RMT.

This study consists in analyzing a SS and a clamped square plate of side length $L=2$ and thickness $h=0.05(\lambda=L / h=40)$ under a uniformly distributed load $q=1$ and a point load $P=4$ acting at the center (Figure 3 ). Isotropic homogeneous material properties are assumed with: $E=0.219, \mu=0.25$, and $G=E / 2(1+\mu)$.

a)

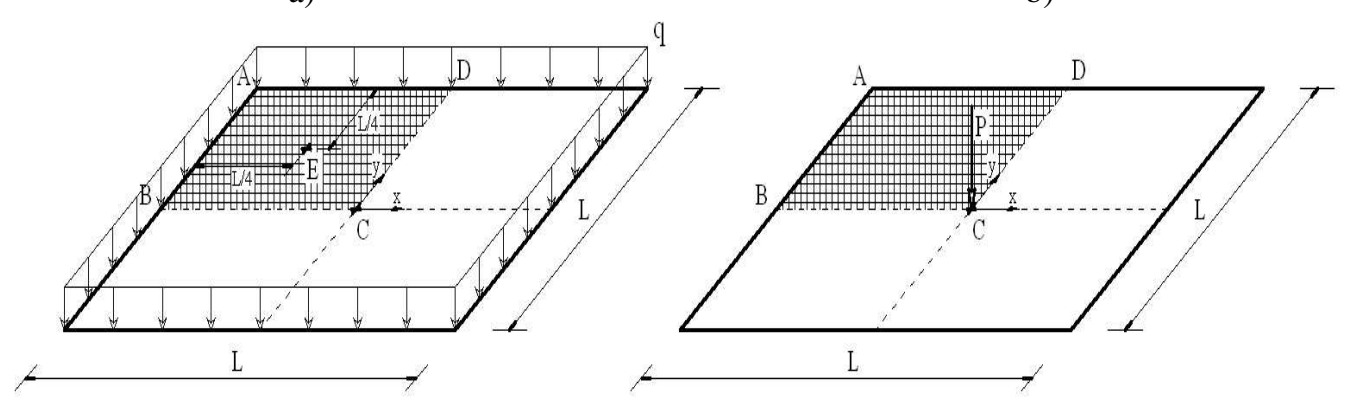

c)

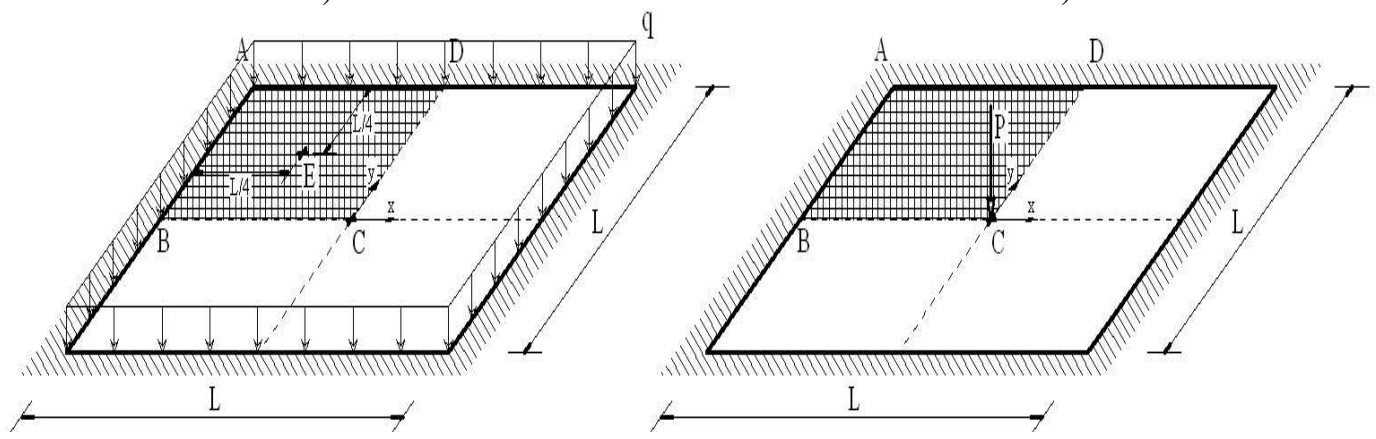

Figure 3 - Square plate ( $\lambda=40$ ) for verification and convergence analysis. SS plate under uniformly distributed load a) and point load b). Clamped plate under uniformly distributed load c) and point load d). 
Assuming symmetry along both axes, only one quarter of the plate is analyzed. Five different meshes of QLRZ elements (figure 4) whose properties are listed in table 2 are employed.

a)

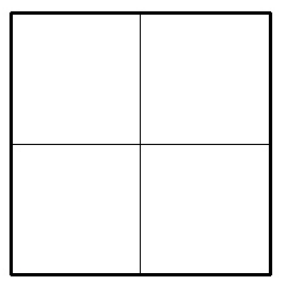

b)

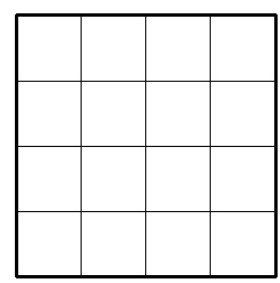

c)

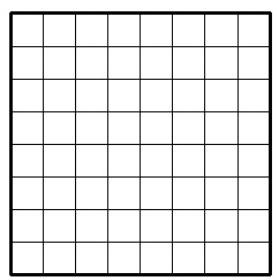

d)

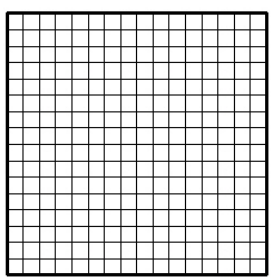

e)

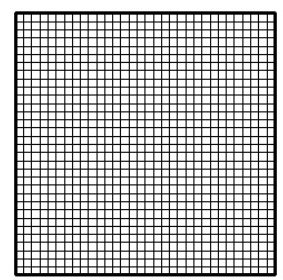

Figure 4 - Meshes of NxN QLRZ elements employed for verification and convergence analysis. a) $\mathrm{N}=$ 2 ; b) $\mathrm{N}=4$; c) $\mathrm{N}=8$; d) $\mathrm{N}=16$; e) $\mathrm{N}=32$.

\begin{tabular}{|c|c|c|c|c|}
\hline \multicolumn{5}{|c|}{ Meshes Properties } \\
\hline Mesh & N & Elements & Nodes & DOFs \\
\hline $\mathbf{1}$ & 2 & 4 & 9 & 45 \\
\hline $\mathbf{2}$ & 4 & 16 & 25 & 150 \\
\hline $\mathbf{3}$ & 8 & 64 & 81 & 405 \\
\hline $\mathbf{4}$ & 16 & 256 & 289 & 1445 \\
\hline $\mathbf{5}$ & 32 & 1024 & 1089 & 5445 \\
\hline
\end{tabular}

Table 2 - QLRZ meshes properties.

In order to asses the element accuracy, the following relative error is defined as

$$
e_{r_{i}}=\frac{w_{i}-w_{K}}{w_{K}}
$$

where $w_{i}$ is the vertical deflection at the center point computed with the $i$ th mesh $(i=1,2, \ldots, 5)$ and $w_{K}$ is the analytical Kirchhoff solution defined as

$$
w_{K}=\alpha \cdot \frac{F \cdot L^{n}}{D} \quad \text { with } \quad D=\frac{E \cdot h^{3}}{12 \cdot\left(1-\mu^{2}\right)}
$$

where $F$ denotes the load and $\alpha$ is a coefficient dependent on the boundary conditions and the load. The analytical solutions of the problem are shown in Table 3.

\begin{tabular}{|c|c|c|c|c|c|}
\hline \multicolumn{5}{|c|}{ Analytical Kirchhoff solutions } \\
\hline Boundary & Load & A & F & n & w (Kirchhoff) \\
\hline \multirow{2}{*}{ SS } & Distributed & 0,00406 & $\mathrm{q}$ & 4 & $\mathbf{0 . 0 2 6 7 1 1 0 1}$ \\
\cline { 2 - 6 } & Point & 0,01160 & $\mathrm{P}$ & 2 & $\mathbf{0 . 0 7 6 2 7 3 9 7}$ \\
\hline \multirow{2}{*}{ Clamped } & Distributed & 0,00122 & $\mathrm{q}$ & 4 & $\mathbf{0 . 0 0 8 2 8 4 9 3}$ \\
\cline { 2 - 6 } & Point & 0,00560 & $\mathrm{P}$ & 2 & $\mathbf{0 . 0 3 6 8 2 1 9 2}$ \\
\hline
\end{tabular}

Table 3 - Analytical Kirchhoff solutions.

The QLRZ solution of the problem and the relative error are presented in Table 4. Figure 5 shows the behavior of the error. Labels SS-P, SS-q, C-P, and C-q in Figure 5 
refer to simply-supported-point-load, simply-supported-distributed-load, clamped-pointload, and clamped-distributed-load, respectively.

\begin{tabular}{|c|c|c|c|c|c|}
\hline \multicolumn{6}{|c|}{ Relative error $\left(e_{r} \%\right)$ of $w$ at center point } \\
\hline \multirow{2}{*}{ Load } & \multirow{2}{*}{ Mesh } & \multicolumn{2}{|c|}{ SS } & \multicolumn{2}{|c|}{ Clamped } \\
\hline & & $\mathbf{w}$ & $\operatorname{er}(\%)$ & $\mathbf{w}$ & $\operatorname{er}(\%)$ \\
\hline \multirow{5}{*}{ 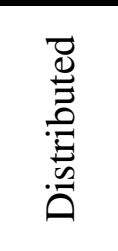 } & $2 \times 2$ & 0.026150 & -2.100 & 0.0080239 & -3.150 \\
\hline & $4 \times 4$ & 0.026638 & -0.273 & 0.0082998 & 0.179 \\
\hline & $8 \times 8$ & 0.026744 & 0.123 & 0.0083747 & 1.083 \\
\hline & $16 \times 16$ & 0.026770 & 0.220 & 0.0083939 & 1.315 \\
\hline & $32 \times 32$ & 0.026776 & 0.243 & 0.0083988 & 1.374 \\
\hline \multirow{5}{*}{$\begin{array}{l}\vec{\Xi} \\
\stackrel{0}{0} \\
.\end{array}$} & $2 \times 2$ & 0.076049 & -0.294 & 0.0322470 & -12.424 \\
\hline & $4 \times 4$ & 0.076392 & 0.154 & 0.0360900 & -1.987 \\
\hline & $8 \times 8$ & 0.076767 & 0.646 & 0.0371910 & 1.002 \\
\hline & $16 \times 16$ & 0.076966 & 0.907 & 0.0375650 & 2.018 \\
\hline & $32 \times 32$ & 0.077097 & 1.079 & 0.0377400 & 2.493 \\
\hline
\end{tabular}

Table 4 - Relative error $e_{r}$ of $w$ at center point.

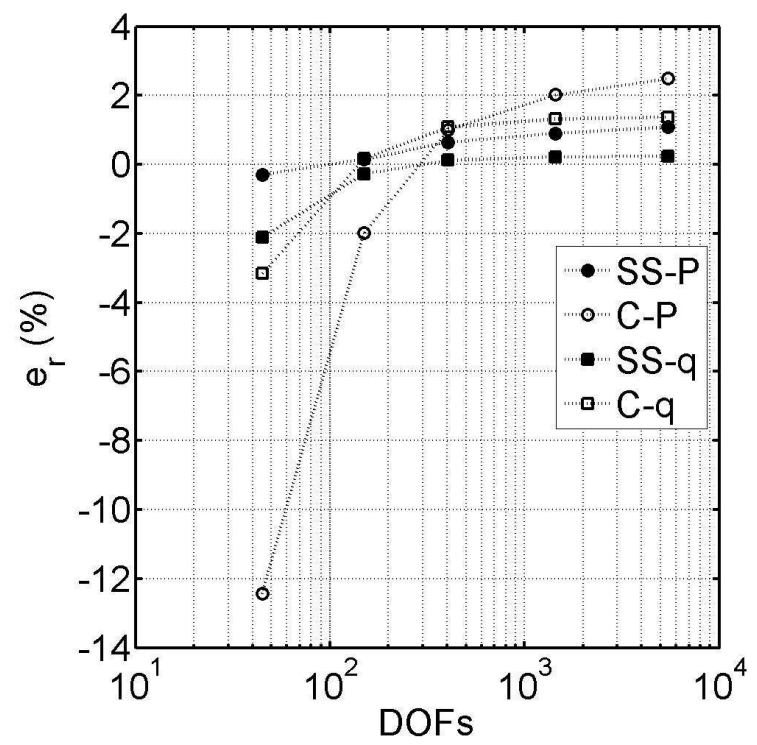

Figure 5 - Relative error $e_{r}$ of central deflection $w$ values.

Table 5 shows that the converged solution is obtained for the $8 \times 8$ mesh $\left(\mathrm{N}^{\circ} 3\right)$. Good accuracy is obtained already for the $4 \times 4$ mesh ( $e_{r}$ less than $2.5 \%$ ). Results for the SS case (error $1.0 \%$ ) are better than for the clamped one. The worst result is obtained for the clamped plate under central point load for the $2 \times 2$ mesh $\left(e_{r}=-12.42 \%\right)$.

\section{CONVERGENCE STUDIES}

We study next the influence of the heterogeneity of the composite material on the convergence and accuracy of the QLRZ element, a SS and clamped square plates of length side $L=2 m$ and thickness $h=0.1 m(\lambda=20)$ under uniformly distributed load $q=1 \mathrm{~N} / \mathrm{m}^{2}$ (Figures $3 \mathrm{a}$ and $3 \mathrm{c}$ ). Three different composite laminated materials, whose 
properties are shown in Table 5, are considered for each example. The degree of heterogeneity increases from composite $\mathrm{C} 1$ to $\mathrm{C} 3$.

\begin{tabular}{|c|c|c|c|c|}
\hline & Properties & $\begin{array}{c}\text { Layer 1 } \\
\text { (Top) }\end{array}$ & Layer 2 & $\begin{array}{c}\text { Layer 3 } \\
\text { (Bottom) }\end{array}$ \\
\hline \multirow{3}{*}{ Composite C1 } & $h[m]$ & $\mathrm{h} / 3$ & $\mathrm{~h} / 3$ & $\mathrm{~h} / 3$ \\
\cline { 2 - 5 } & $E[M P a]$ & 0.219 & $0.219 \times 10^{-1}$ & 0.44 \\
\cline { 2 - 5 } & $\mu$ & 0.25 & 0.25 & 0.25 \\
\hline \multirow{3}{*}{ Composite C2 } & $h[m]$ & $\mathrm{h} / 3$ & $\mathrm{~h} / 3$ & $\mathrm{~h} / 3$ \\
\cline { 2 - 5 } & $E[M P a]$ & 0.219 & $0.219 \times 10^{-2}$ & 0.219 \\
\cline { 2 - 5 } Composite C3 & $\mu$ & 0.25 & 0.25 & 0.25 \\
\cline { 2 - 5 } & $E[m]$ & $\mathrm{h} / 10$ & $\mathrm{~h} / 1.25$ & $\mathrm{~h} / 10$ \\
\cline { 2 - 5 } & $\mu$ & 0.219 & $0.725 \times 10^{-3}$ & $0.73 \mathrm{E} \times 10^{-1}$ \\
\hline
\end{tabular}

Table 5 - Composite material properties.

Taking advantage of symmetry only one quarter of plate is analyzed using the QLRZ meshes described in section 5 (Figure 4). The reference solution was obtained by a 3D finite element analysis using a mesh of 10x10x9 (3 elements per ply) 20-noded hexahedral elements involving 4499 nodes and 13497 DOFs (Figure 6).

a)
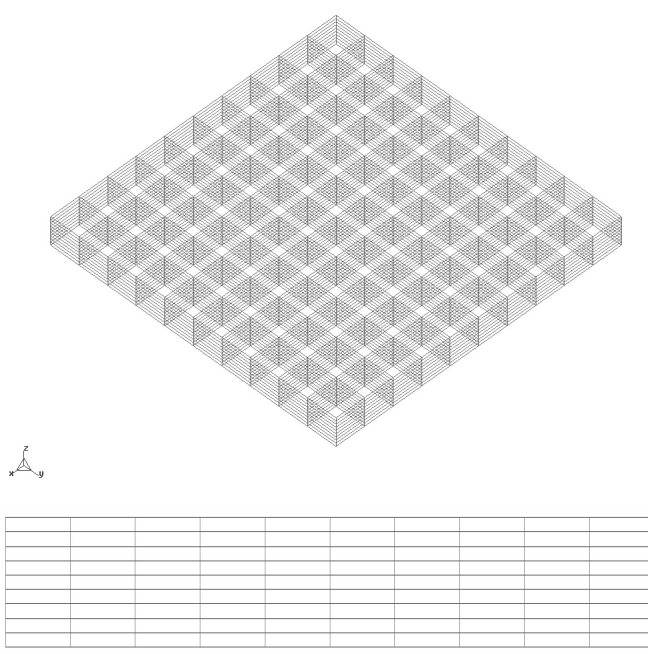

b)

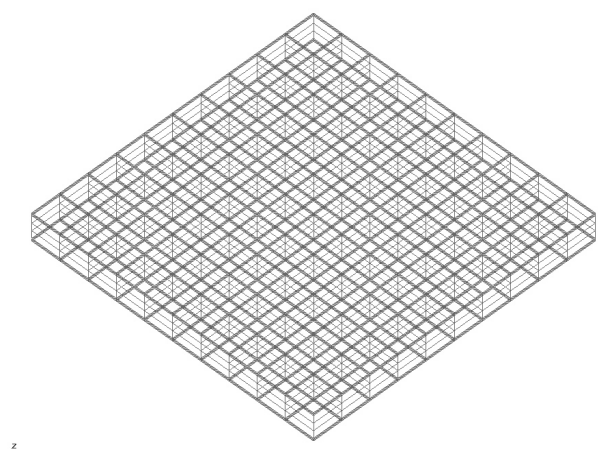

$x_{y}^{2}$

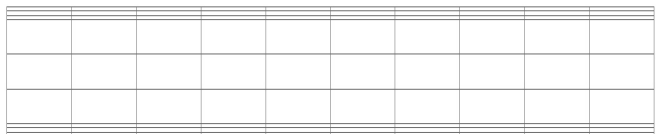

$\alpha$.

Figure 6 - 10x10x9 HEXA20 meshes employed to compute the reference solution for composite $\mathrm{C} 1$ and $\mathrm{C} 2 \mathrm{a}$ ), and composite $\mathrm{C} 3 \mathrm{~b}$ ).

Convergence is quantified by the relative error defined as

$$
e_{r}=\frac{m_{i}-m_{3 D}}{m_{3 D}}
$$


where $m_{i}$ and $m_{3 D}$ are the magnitudes of interest obtained with the $i$ th QLRZ mesh $(i=1,2, \ldots, 5)$ and the $3 \mathrm{D}$ reference solution, respectively. The magnitudes studied $m$ are: the vertical deflection $w$ at the center point $\mathrm{C}$ (Figure 3), the axial stress $\sigma_{x}$ on the top surface of ply 1 at point E, and $\psi_{x}$ at point E. Since $\psi_{x}$ does not appear in 3D finite element analysis, $m_{i}$ and $m_{3 D}$ are the values of this magnitude obtained using the $i$ th QLRZ mesh $(i=1, \ldots, 4)$ and the finest mesh (32x32), respectively. The results obtained are shown in Tables 6-7, and Figures 7-8.

It is clearly seen that convergence is always slower for the more heterogeneous material and for the clamped plate.

For the clamped plate and the three materials (Table 6) errors are less than $10 \%$ for the $16 \times 16$ mesh for all variables. For the SS plate (Table 7) errors are less than $2.3 \%$ for the $8 \times 8$ mesh in all cases.

For composite $\mathrm{C} 1$ (the more homogeneous one) errors are less than $2.9 \%$ for the $8 \mathrm{x} 8$ mesh in all cases and less than $6.3 \%$ for the $4 \times 4$ mesh in all cases except for $\sigma_{x}$ in the clamped plate.

For the more heterogeneous material (composite C3), the difference in the results between the SS and the clamped plate is larger. For the SS plate (Table 7) errors are less than $2.3 \%$ for the $8 \times 8$ mesh in all variables. For the clamped plate (Table 6) errors are less than $23 \%$ for the $8 \times 8$ mesh and less than $10 \%$ for the $16 \times 16$ mesh in all cases.

The quality of the results obtained for the composite $\mathrm{C} 2$ are between that of composites $\mathrm{C} 1$ and $\mathrm{C} 3$.

\begin{tabular}{|c|c|c|c|c|c|c|c|c|c|}
\hline \multicolumn{8}{|c|}{ Relative error $e_{r}(\%)$ in clamped plate } \\
\hline \multirow{2}{*}{ Mesh } & \multicolumn{3}{|c|}{ w at point C } & \multicolumn{3}{|c|}{$\boldsymbol{\sigma}_{\mathbf{x}}$ at point $\mathrm{E}$} & \multicolumn{3}{|c|}{$\boldsymbol{\psi}_{\mathbf{x}}$ at point $\mathrm{E}$} \\
\cline { 2 - 11 } & $\mathbf{C 1}$ & $\mathbf{C 2}$ & $\mathbf{C 3}$ & $\mathbf{C 1}$ & $\mathbf{C 2}$ & $\mathbf{C 3}$ & $\mathbf{C 1}$ & $\mathbf{C 2}$ & $\mathbf{C 3}$ \\
\hline $2 \times 2$ & 11,71 & 50,28 & 60,99 & 99,99 & 100 & 100 & 26,13 & 80,09 & 86,48 \\
\hline $4 \times 4$ & 4,65 & 30,16 & 43,47 & 20,86 & 44,14 & 45,53 & $-6,28$ & 43,34 & 54,80 \\
\hline $8 \times 8$ & 1,60 & 12,32 & 22,44 & 2,90 & 14,35 & 17,24 & $-1,47$ & 13,68 & 18,58 \\
\hline $16 \times 16$ & 0,29 & 3,67 & 9,25 & $-1,21$ & $-0,40$ & $-1,15$ & $-0,30$ & 2,58 & 2,22 \\
\hline $32 \times 32$ & $-0,14$ & 0,69 & 2,85 & $-2,22$ & $-4,70$ & $-4,62$ & 0,00 & 0,00 & 0,00 \\
\hline
\end{tabular}

Table 6 - Clamped square plate ( $\lambda=20$ ) under uniformly distributed load. Relative error $e_{r}(\%)$ for $w$,

$$
\sigma_{x}, \text { and } \psi_{x} \text {. }
$$

\begin{tabular}{|c|c|c|c|c|c|c|c|c|c|}
\hline \multicolumn{10}{|c|}{ Relative error $e_{r}(\%)$ in SS plate } \\
\hline \multirow{2}{*}{ Mesh } & \multicolumn{3}{|c|}{ w at point C } & \multicolumn{3}{c|}{$\boldsymbol{\sigma}_{\mathbf{x}}$ at point $\mathrm{E}$} & \multicolumn{3}{|c|}{$\boldsymbol{\Psi}_{\mathbf{x}}$ at point $\mathrm{E}$} \\
\cline { 2 - 11 } & $\mathbf{C 1}$ & $\mathbf{C 2}$ & $\mathbf{C 3}$ & $\mathbf{C 1}$ & $\mathbf{C 2}$ & $\mathbf{C 3}$ & $\mathbf{C 1}$ & $\mathbf{C 2}$ & $\mathbf{C 3}$ \\
\hline $2 \times 2$ & 2,69 & 19,36 & 25,83 & 26,98 & 32,89 & 33,24 & $-9,11$ & 41,06 & 51,92 \\
\hline $4 \times 4$ & 0,68 & 6,50 & 10,14 & 4,86 & 7,70 & 9,05 & $-3,99$ & 8,95 & 13,67 \\
\hline $8 \times 8$ & 0,25 & 1,54 & 2,22 & $-0,30$ & $-0,79$ & 0,44 & $-0,71$ & $-0,40$ & $-1,84$ \\
\hline $16 \times 16$ & 0,15 & 0,38 & 0,35 & $-1,55$ & $-3,04$ & $-1,92$ & 0,07 & $-0,45$ & $-1,44$ \\
\hline $32 \times 32$ & 0,12 & 0,12 & $-0,02$ & $-1,86$ & $-3,49$ & $-2,07$ & 0,00 & 0,00 & 0,00 \\
\hline
\end{tabular}

Table 7 - SS square plate ( $\lambda=20$ ) under uniformly distributed load. Relative error $e_{r}(\%)$ for $w, \sigma_{x}$, and $\psi_{x}$. 
(a)

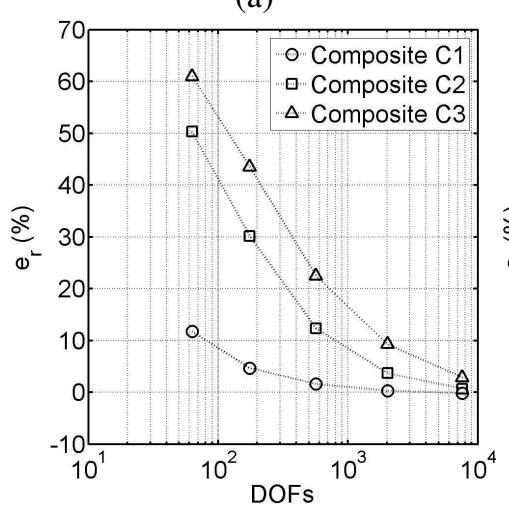

(b)

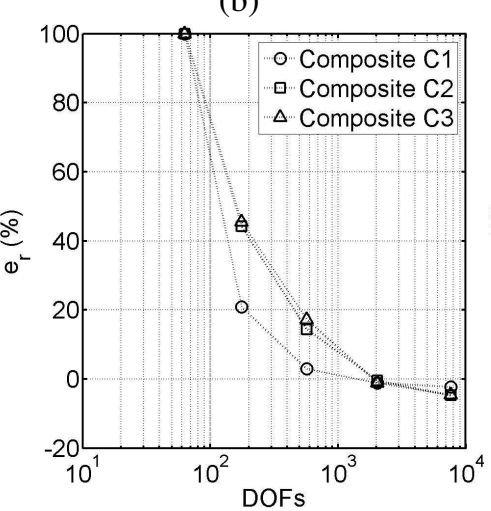

(c)

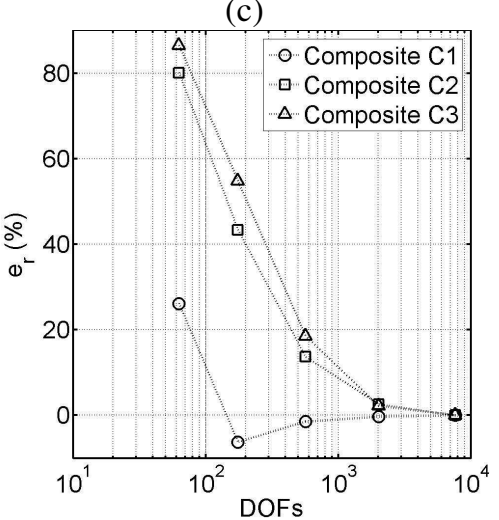

Figure 7 - Clamped square plate ( $\lambda=20$ ) under uniformly distributed load. Relative error $e_{r}(\%)$ for $w$ a), $\sigma_{x}$ b), and $\psi_{x}$ c).

(a)

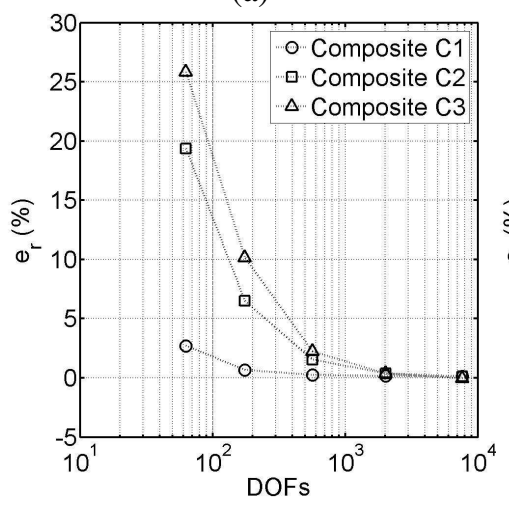

(b)

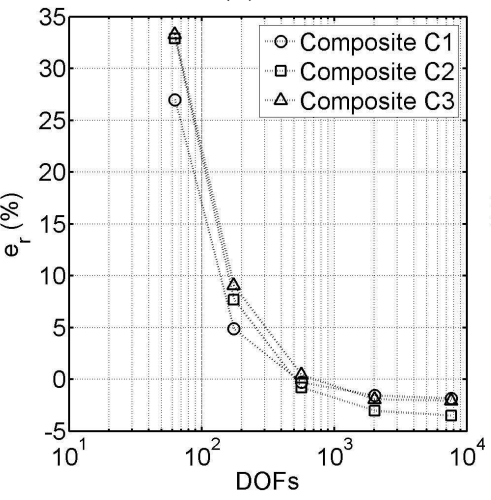

(c)

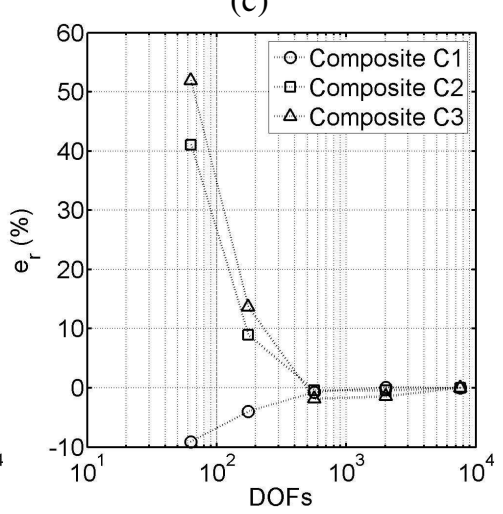

Figure 8 - SS square plate ( $\lambda=20$ ) under uniformly distributed load. Relative error $e_{r}(\%)$ for $w$ a), $\sigma_{x}$ b), and $\psi_{x}$ c).

\section{COMPARISON STUDIED FOR SS SQUARE AND CIRCULAR COMPOSITE LAMINATED PLATES}

In order to show the performance of the QLRZ element for highly heterogeneous composite material, a square SS plate of length $L=2 m$ and thickness $h=0.1 \mathrm{~m}$, and a circular SS plate of diameter $d=2 m$ and thickness $h=0.1 m$ are studied. The structures are loaded under a uniformly distributed load, $q=10000 \mathrm{~N} / \mathrm{m}^{2}$ (Figure 9).

Each plate is studied for different composite laminated materials with properties shown in Tables 8 and 9. The square plate is analyzed for composites C4-7 and the circular plate for composites C6-7.

Do to symmetry only one quarter of plate is analyzed using the QLRZ meshes shown in Figure 10 whose properties are listed in Table 10. The reference solution is the 3D finite element analysis using HEXA20 elements. The different 3D meshes for each case are shown in Figure 11. Details of each mesh are given in Table 11.

The RMT results for the square plate of composite C4 are also shown in Figure 12. The RMT solution was obtained by using a mesh of 16x16 four-noded QLLL plate element $[13,14]$ 
a)

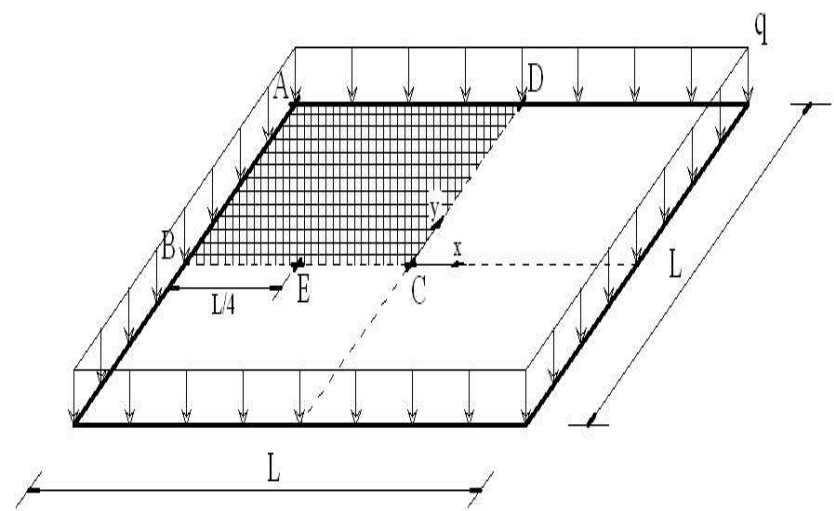

b)

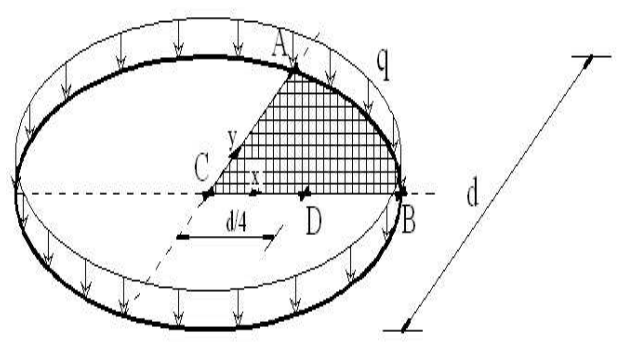

Figure 9 - Square SS plate a) and circular SS plate b) under uniformly distributed load.

\begin{tabular}{|c|c|c|c|c|}
\hline \multicolumn{5}{|c|}{ Layer material properties } \\
\hline & $\bar{A}$ & $\mathrm{~B}$ & $\mathrm{C}$ & $\bar{D}$ \\
\hline $\mathbf{E}_{1}$ & $157.9 \times 10^{2}$ & 19.15 & \multirow{3}{*}{$0.104 \times 10^{2}$} & \multirow{3}{*}{$104.1 \times 10^{2}$} \\
\hline $\mathbf{E}_{2}$ & $9.58 \times 10^{2}$ & 19.15 & & \\
\hline $\mathbf{E}_{3}$ & $9.58 \times 10^{2}$ & 191.5 & & \\
\hline$\mu_{12}$ & 0.32 & $6.58 \times 10^{-4}$ & \multirow{3}{*}{0.30} & \multirow{3}{*}{0.31} \\
\hline$\mu_{13}$ & 0.32 & $6.43 \times 10^{-8}$ & & \\
\hline$\mu_{23}$ & 0.49 & $6.43 \times 10^{-8}$ & & \\
\hline $\mathbf{G}_{12}$ & $5.93 \times 10^{2}$ & $42.3 \times 10^{-7}$ & \multirow{2}{*}{$0.04 \times 10^{2}$} & \multirow{2}{*}{$39.73 \times 10^{2}$} \\
\hline $\mathbf{G}_{13}$ & $5.93 \times 10^{2}$ & 36.51 & & \\
\hline $\mathbf{G}_{23}$ & $3.23 \times 10^{2}$ & 124.8 & & \\
\hline
\end{tabular}

Table 8 - Layer material properties. $\mathrm{E}$ and $\mathrm{G}$ are given in $\mathrm{MPa}$.

\begin{tabular}{|c|c|c|}
\hline \multicolumn{3}{|c|}{ Composite laminated materials } \\
\hline Composite & Layer distribution & $h^{k} / h$ \\
\hline C4 & $(\mathrm{A} / \mathrm{C} / \mathrm{A})$ & $(0.1 / 0.8 / 0.1)$ \\
\hline C5 & $(\mathrm{A} / \mathrm{B})$ & $(0.5 / 0.5)$ \\
\hline C6 & $(\mathrm{A} / \mathrm{B} / \mathrm{C} / \mathrm{D})$ & $(0.1 / 0.3 / 0.5 / 0.1)$ \\
\hline C7 & $(\mathrm{A} / \mathrm{C} / \mathrm{A} / \mathrm{C} / \mathrm{B} / \mathrm{C} / \mathrm{A} / \mathrm{C} / \mathrm{A})$ & $(0.1 / 0.1 / 0.1 / 0.1 / 0.2 / 0.1 / 0.1 / 0.1 / 0.1)$ \\
\hline
\end{tabular}

Table 9 -Layer distribution of the composite materials. 
a)

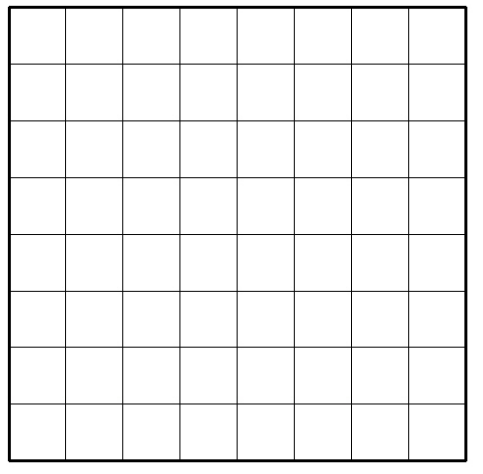

c)

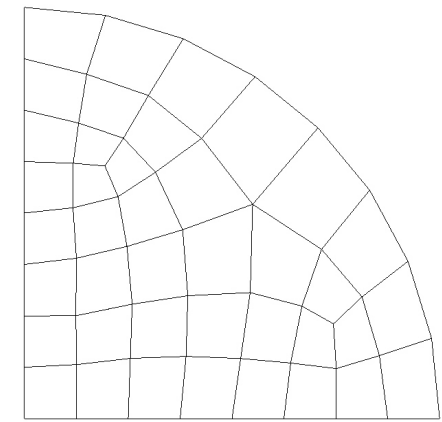

*.

Figure 10 - QLRZ meshes. Square plate: 8x8 a) and 16x16 element b). Circular plate: 40 c) and $168 \mathrm{~d}$ ) elements.

b)

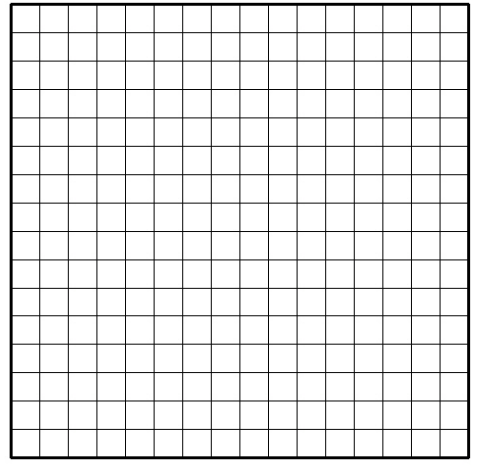

d)

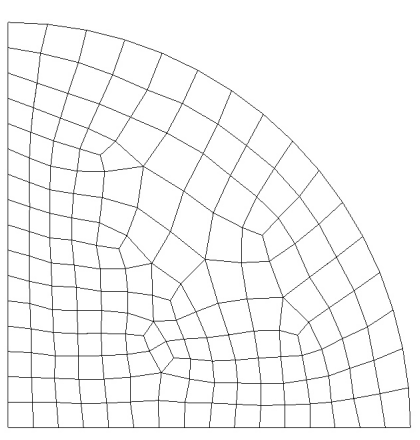


a)
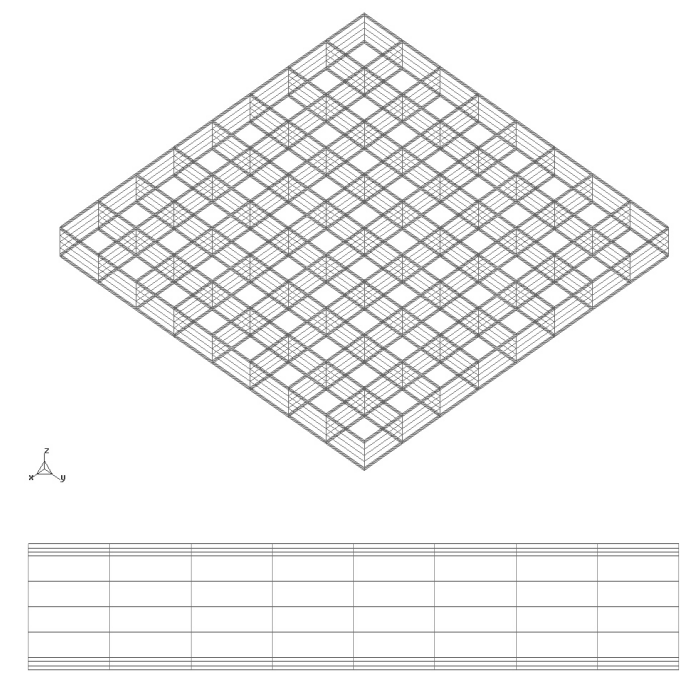

c)
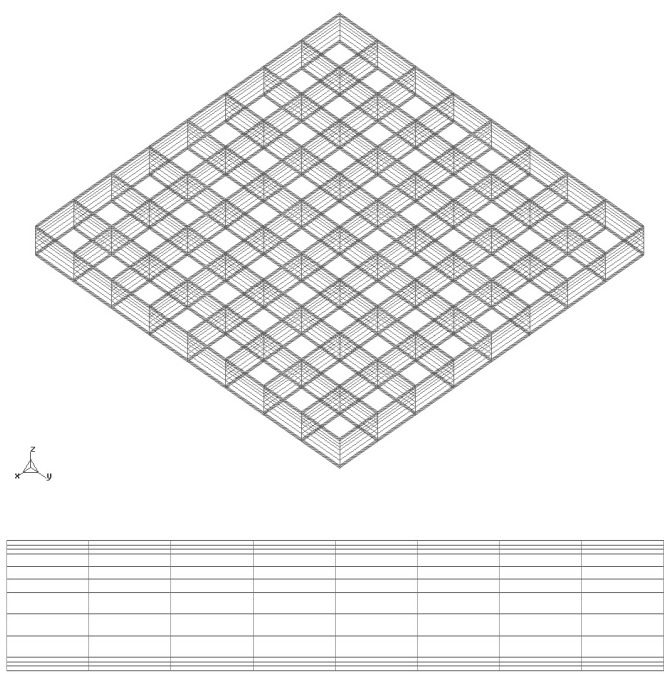

e)

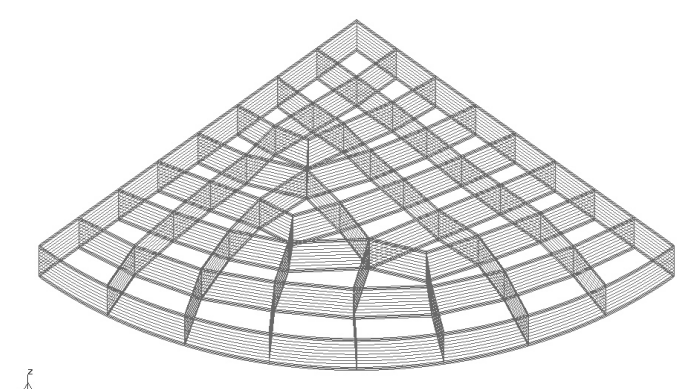
$\AA_{y}^{2}$

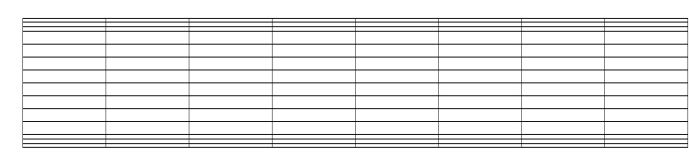

b)
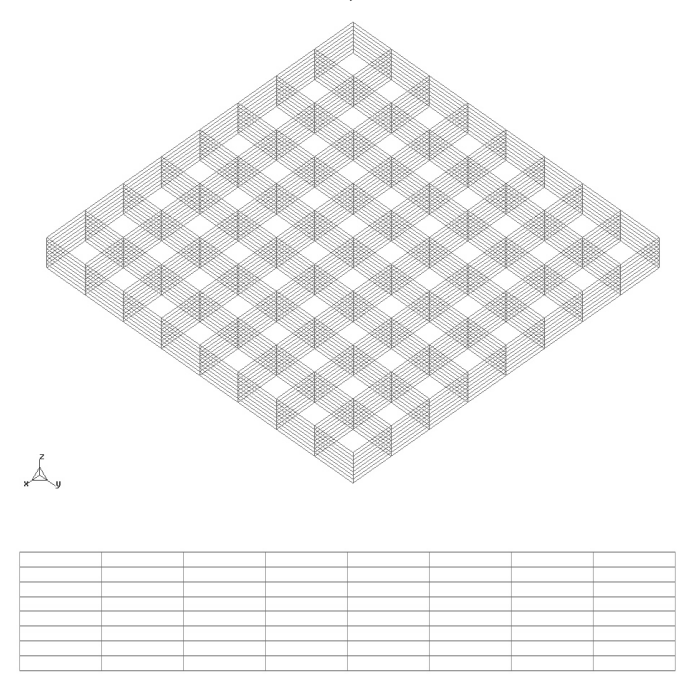

d)
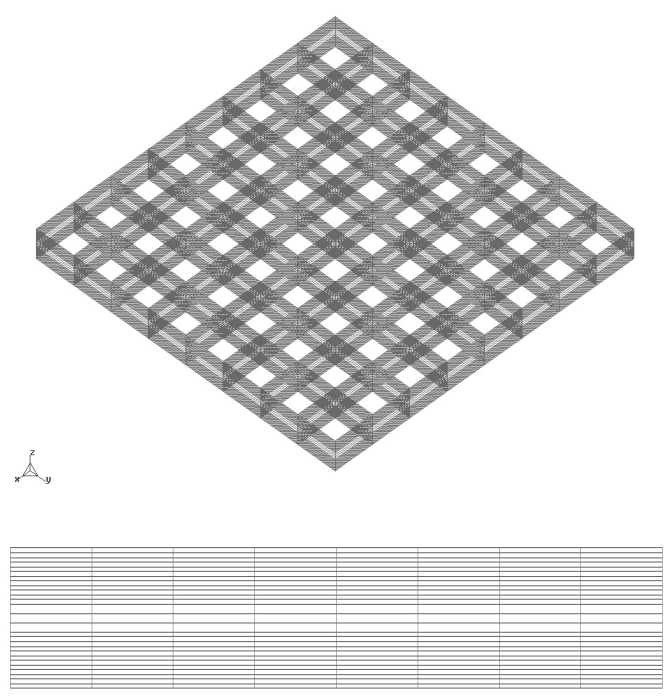

f)
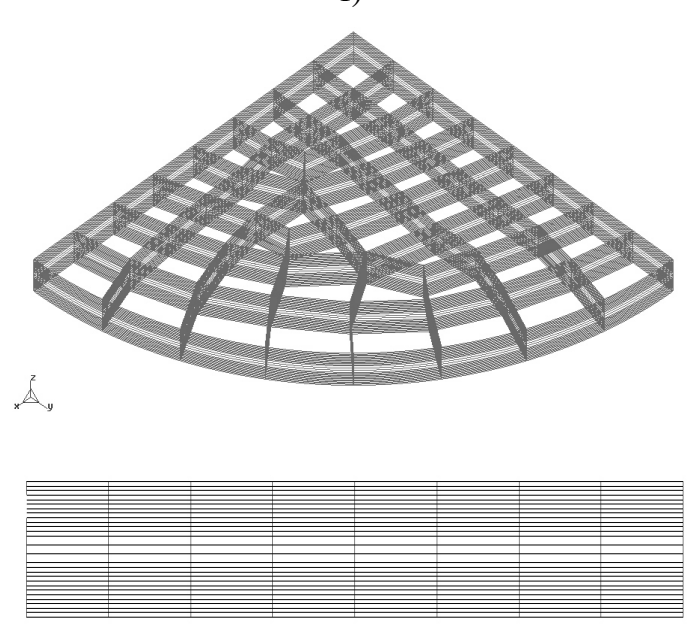

Figure 11 - HEXA20 reference meshes. Square meshes for composites C4 a), C5 b), C6 c), C7 d), and circular meshes for composites C6 e) and C7 f). 


\begin{tabular}{|c|c|c|c|c|}
\hline \multicolumn{5}{|c|}{ QLRZ meshes properties } \\
\hline $\begin{array}{c}\text { Meshes } \\
\text { (Figure 10) }\end{array}$ & NxN & $\begin{array}{c}\text { Number of } \\
\text { elements }\end{array}$ & Nodes & DOFs \\
\hline a & $8 \times 8$ & 64 & 81 & 567 \\
\hline b & $16 \times 16$ & 256 & 289 & 2023 \\
\hline c & -- & 40 & 53 & 371 \\
\hline d & -- & 168 & 193 & 1351 \\
\hline
\end{tabular}

Table 10 - QLRZ mesh properties.

\begin{tabular}{|c|c|c|c|c|}
\hline \multicolumn{5}{|c|}{ HEXA20 mesh properties } \\
\hline $\begin{array}{c}\text { Mesh } \\
\text { (Figure 11) }\end{array}$ & Composite & $\begin{array}{c}\text { Number } \\
\text { of elements }\end{array}$ & Nodes & DOFs \\
\hline a & C4 & 640 & 3285 & 9855 \\
\hline b & C5 & 512 & 2673 & 8019 \\
\hline c & C6 & 768 & 3897 & 11691 \\
\hline d & C7 & 1728 & 8487 & 25461 \\
\hline e & C6 & 602 & 3094 & 9282 \\
\hline f & C7 & 1161 & 5824 & 17472 \\
\hline
\end{tabular}

Table 11 - HEXA20 mesh properties.

Figures 12-17 show the computed vertical deflection $w$ (a), the thickness distribution of the axial displacement $u(\mathrm{~b})$, the axial stress $\sigma_{x}(\mathrm{c})$, the transverse shear stress $\tau_{x z}$ (d) for each plate under study.

The vertical deflection is accurately captured. At the center of plate, the maximum error (14\%) is given by the circular plate of composite C6 using the 40-element mesh (Figure 16a). For the finest mesh (168 elements) the computed errors are less than $10 \%$.

The thickness distribution of the axial displacement is accurately predicted in all cases. The ability to capture the complex kinematics of laminated composite materials is a key feature of the QLRZ plate element. The successful axial displacement prediction leads to accurate axial stress values as shown in Figures c). Figures d) display the good results for the thickness distribution of transverse shear stresses computed a posteriori using Eq.(24).

Results demonstrate the good performance of the QLRZ element.

Figure 12 shows the inaccurate results when modeling a composite laminated plate using QLLL elements based on RMT. The deflection at the plate center is three times stiffer than the reference solution (Figure 12a). The RMT solution also yields an erroneous linear thickness distribution of the axial displacement (Figure 12b), which leads to a distorted distribution of the axial stress (Figure 12c). Finally, the RMT is unable to capture the correct transverse shear stress distribution (Figure 12d). 
a)

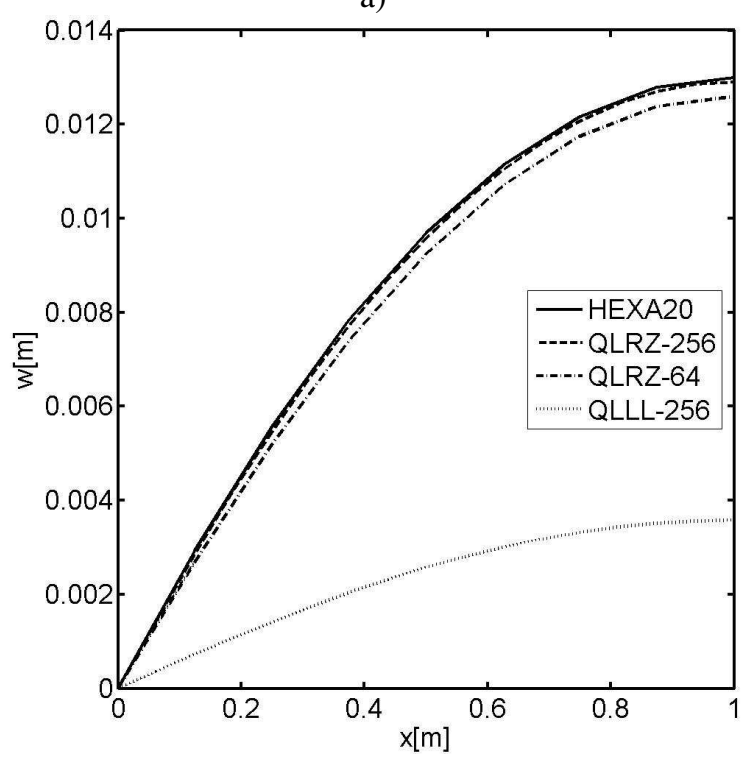

c)

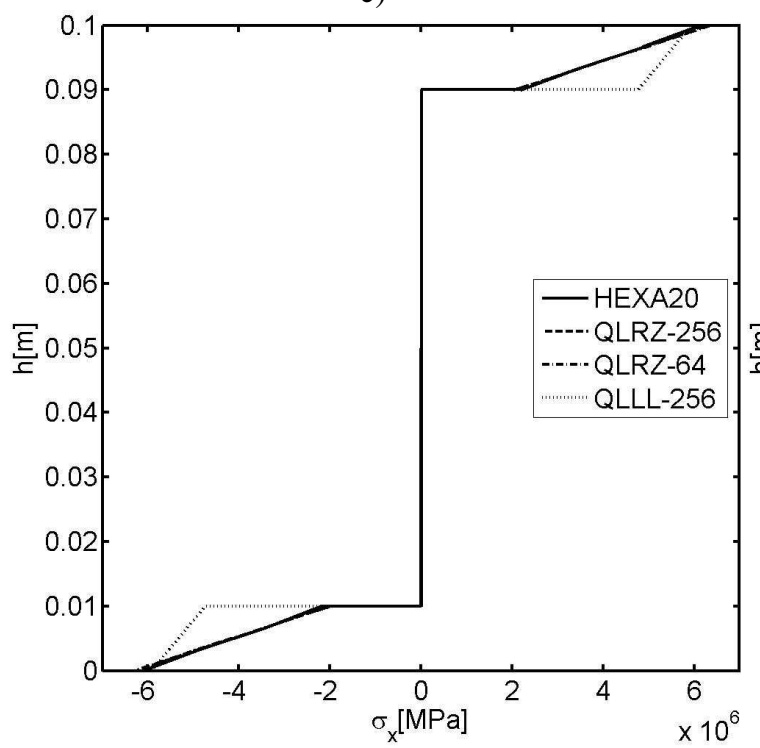

b)

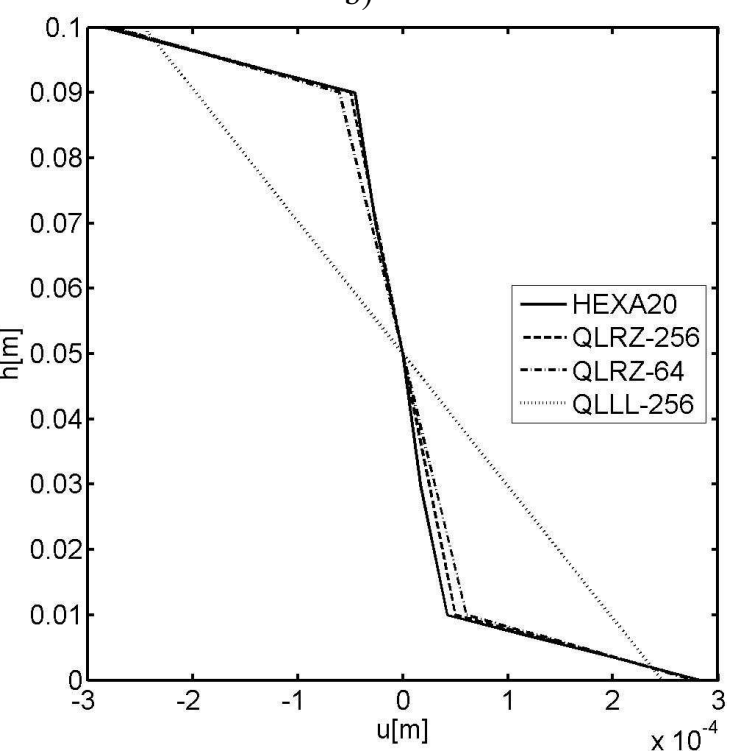

d)

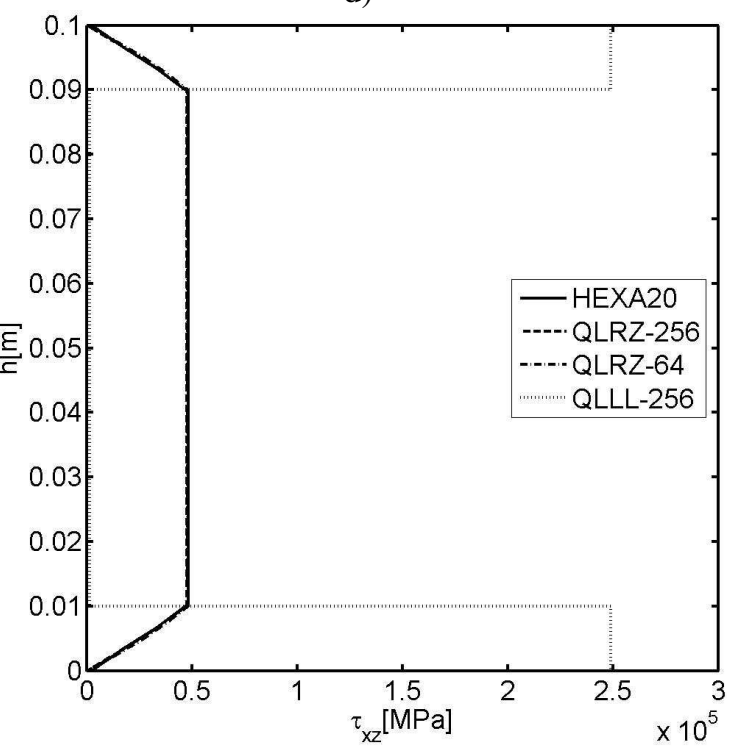

Figure 12 - SS square plate under uniformly distributed load. Composite C4. a) Vertical deflection along central line BC. Thickness distribution of: b) axial displacement $u$ at point B, c) axial stress $\sigma_{x}$ at the center point $\mathrm{C}$, and $\mathrm{d}$ ) transverse shear stress $\tau_{x z}$ at point $\mathrm{E}$. 
a)

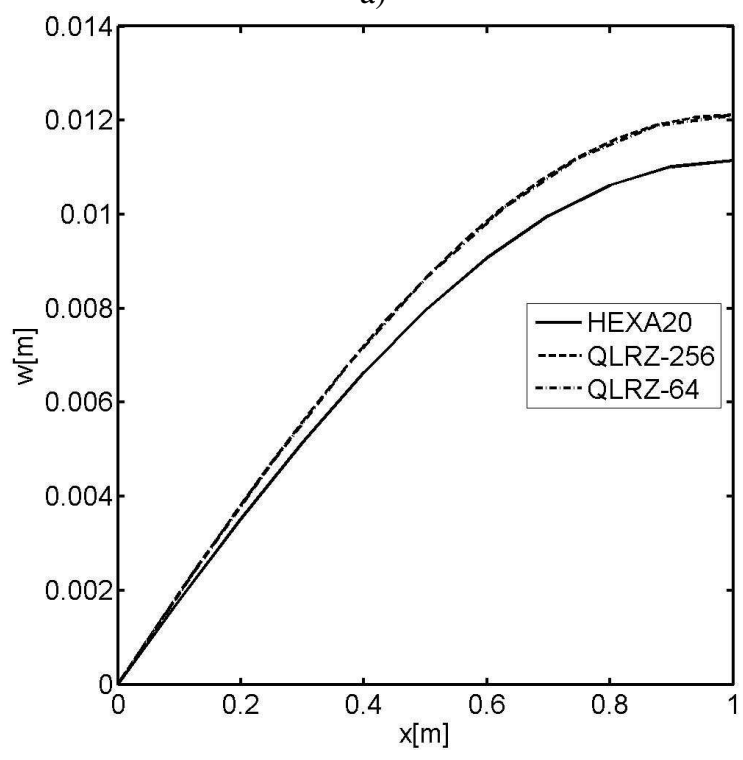

c)

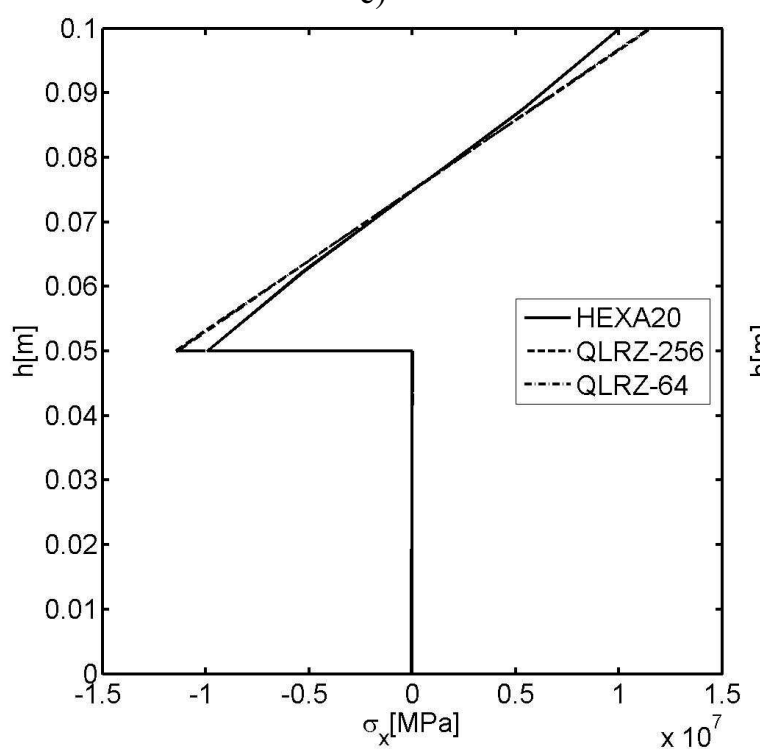

b)

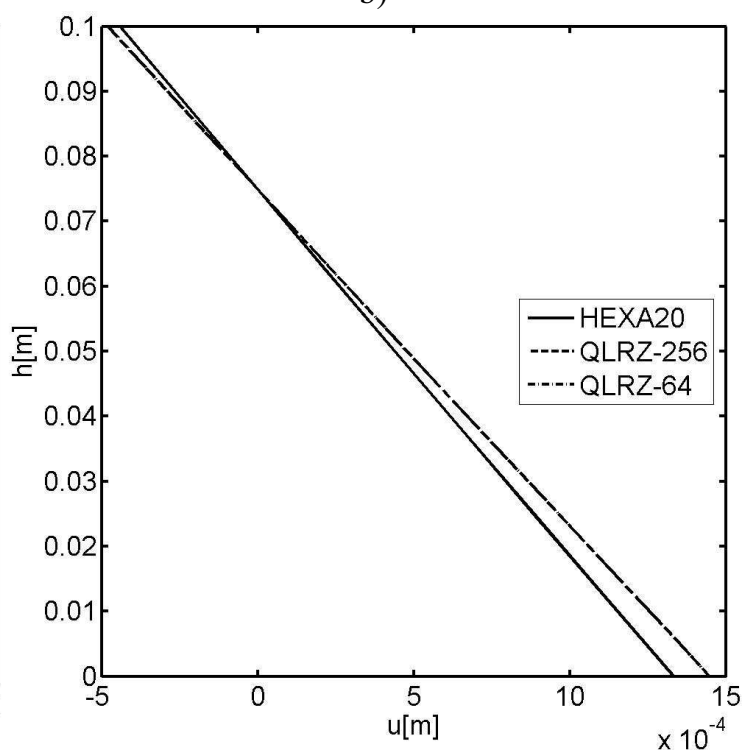

d)

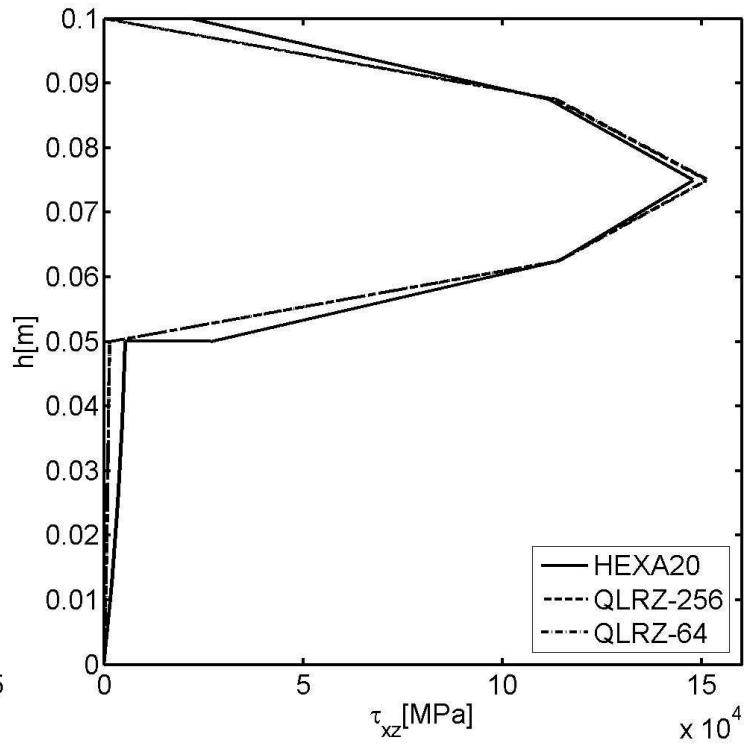

Figure 13 - SS square plate under uniformly distributed load. Composite C5. a) Vertical deflection along central line BC. Thickness distribution of: b) axial displacement $u$ at point $\mathrm{B}, \mathrm{c}$ ) axial stress $\sigma_{x}$ at the center point $\mathrm{C}$, and d) transverse shear stress $\tau_{x z}$ at point $\mathrm{E}$. 
a)

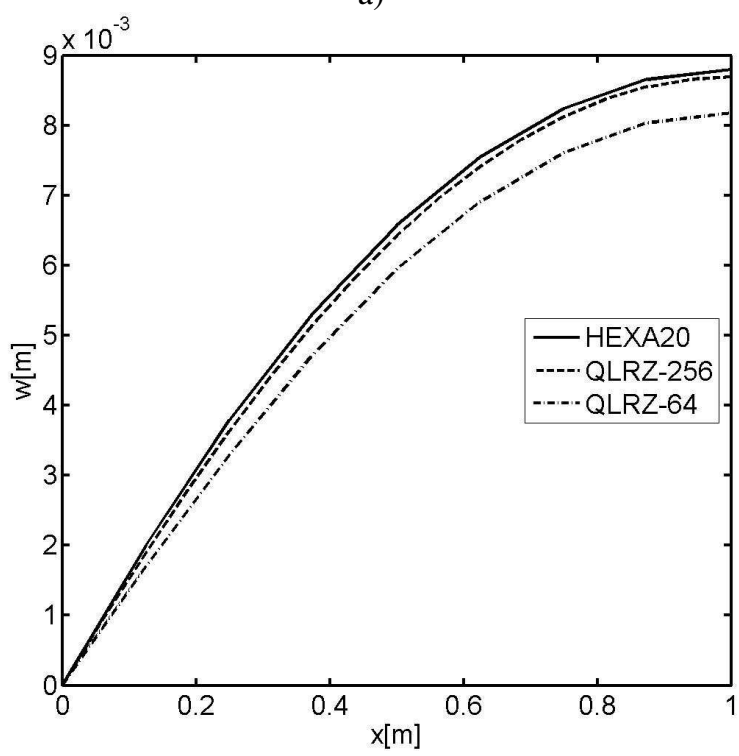

c)

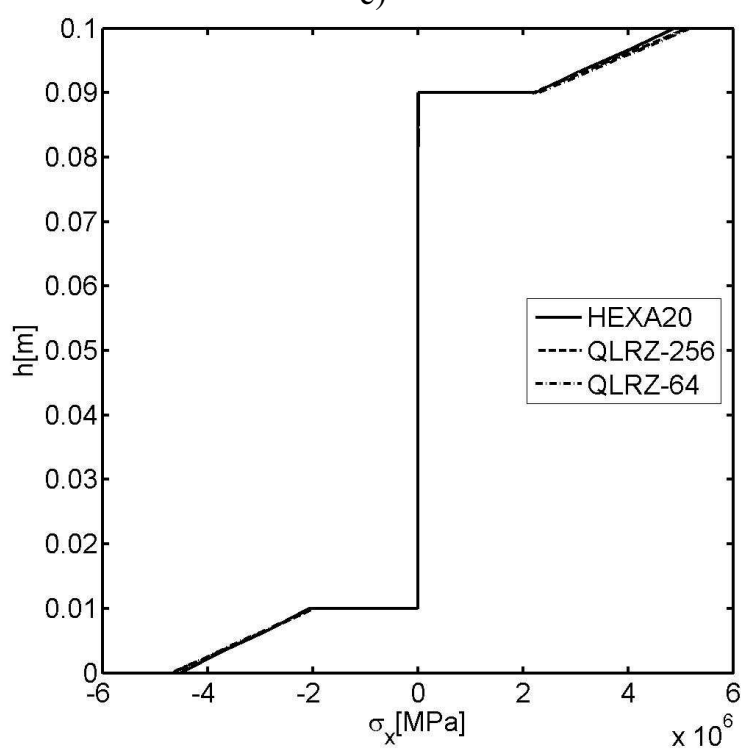

b)

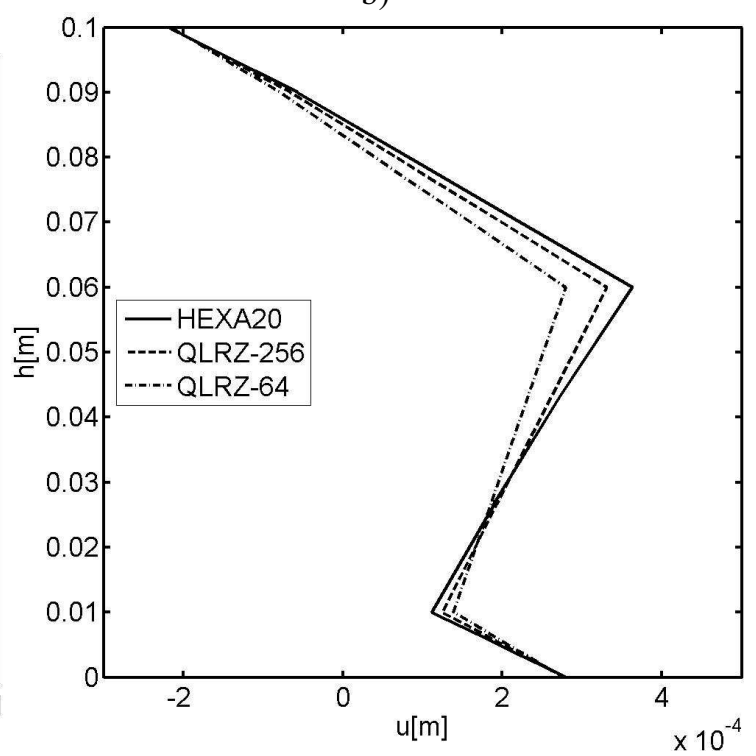

d)

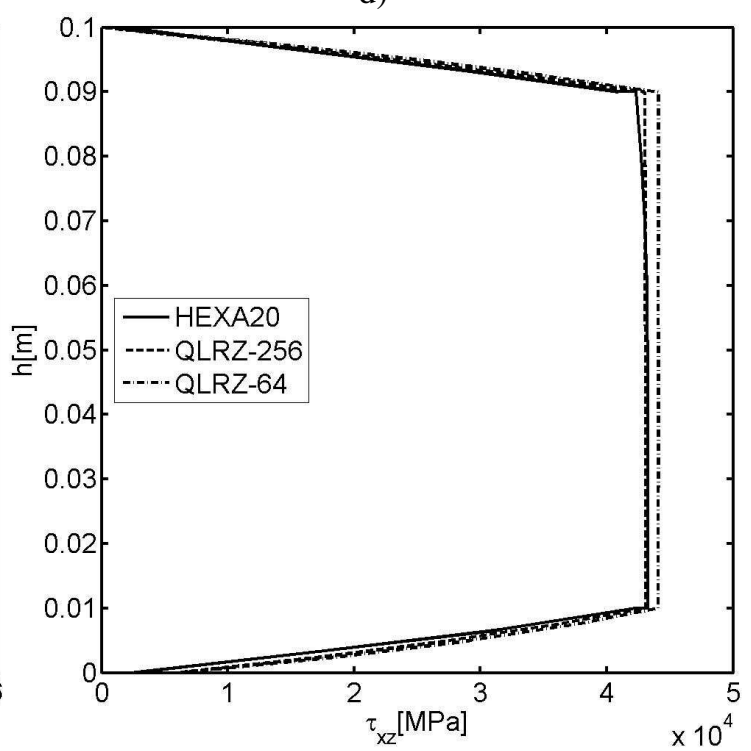

Figure 14 - SS square plate under uniformly distributed load. Composite C6. a) Vertical deflection along central line BC. Thickness distribution of: b) axial displacement $u$ at point B, c) axial stress $\sigma_{x}$ at the center point $\mathrm{C}$, and d) transverse shear stress $\tau_{x z}$ at point $\mathrm{E}$. 
a)

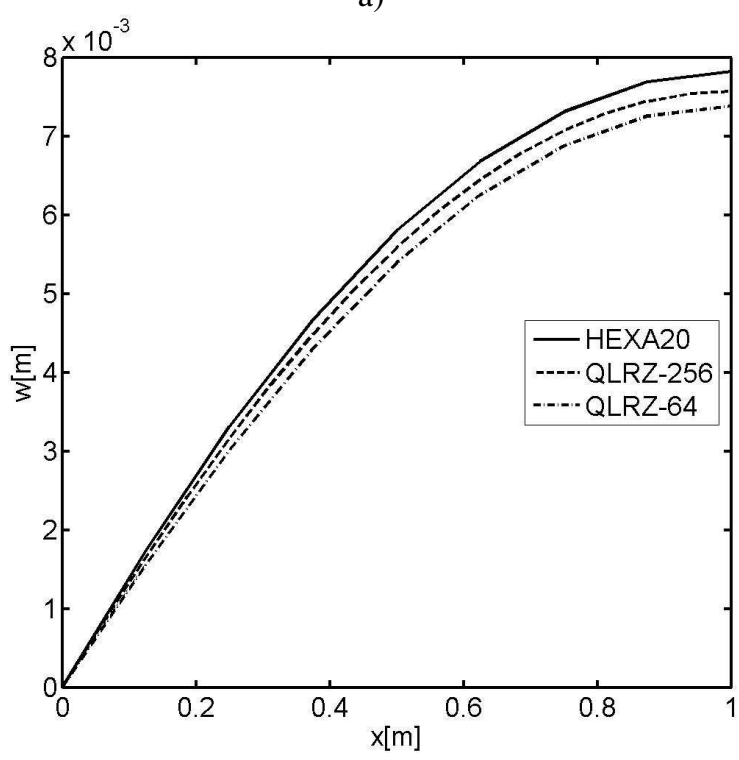

c)

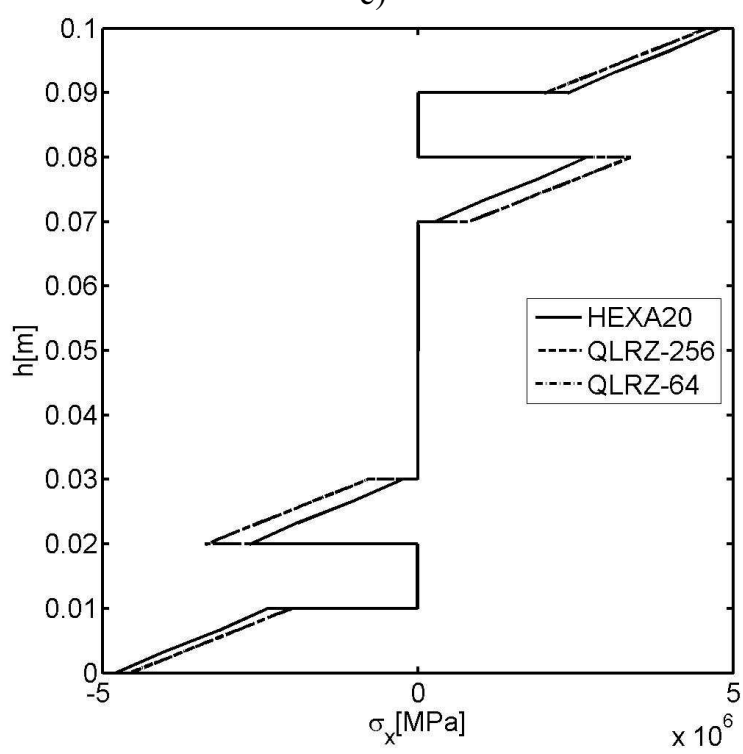

b)

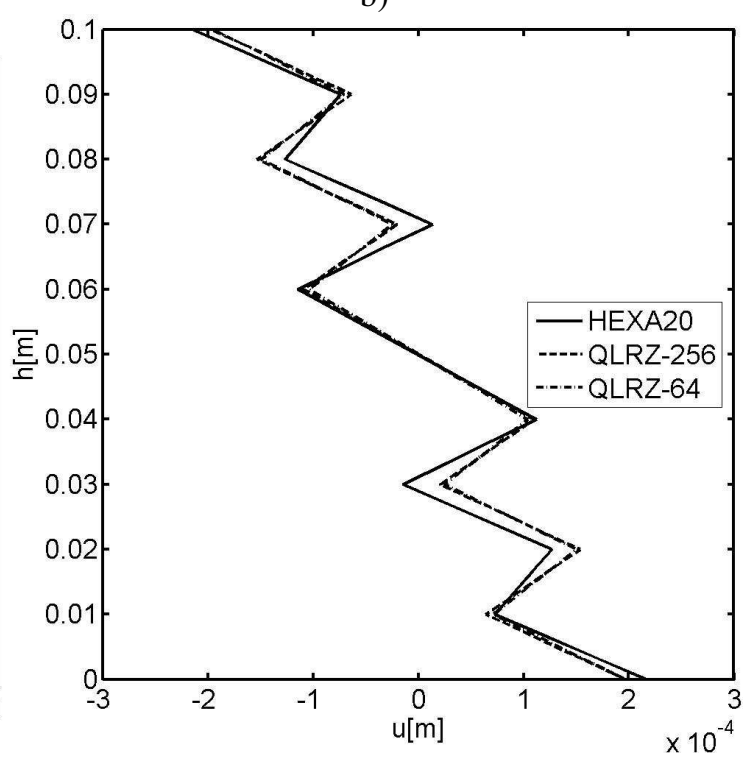

d)

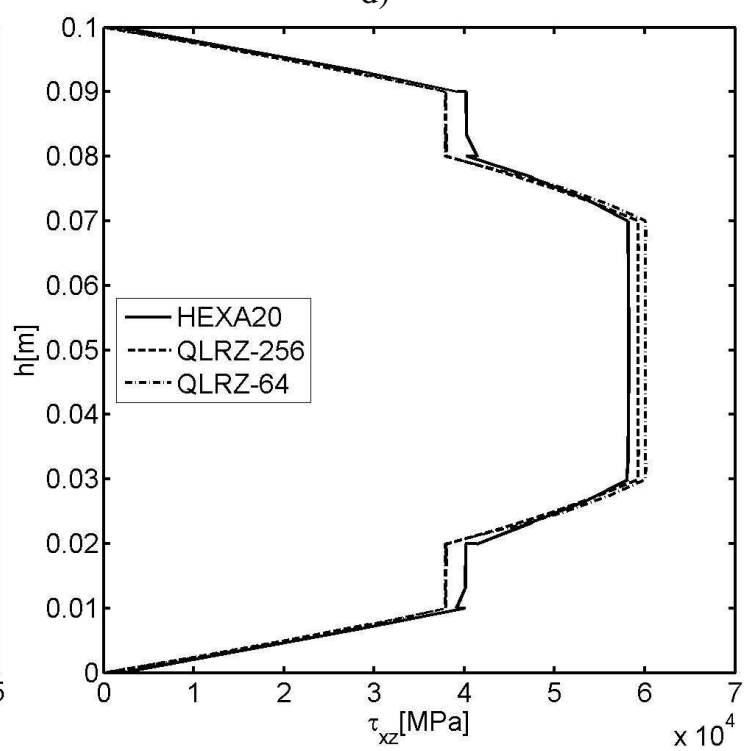

Figure 15 - SS square plate under uniformly distributed load. Composite C7. a) Vertical deflection along central line BC. Thickness distribution of: b) axial displacement $u$ at point B, c) axial stress $\sigma_{x}$ at the center point $\mathrm{C}$, and d) transverse shear stress $\tau_{x z}$ at point $\mathrm{E}$. 
a)

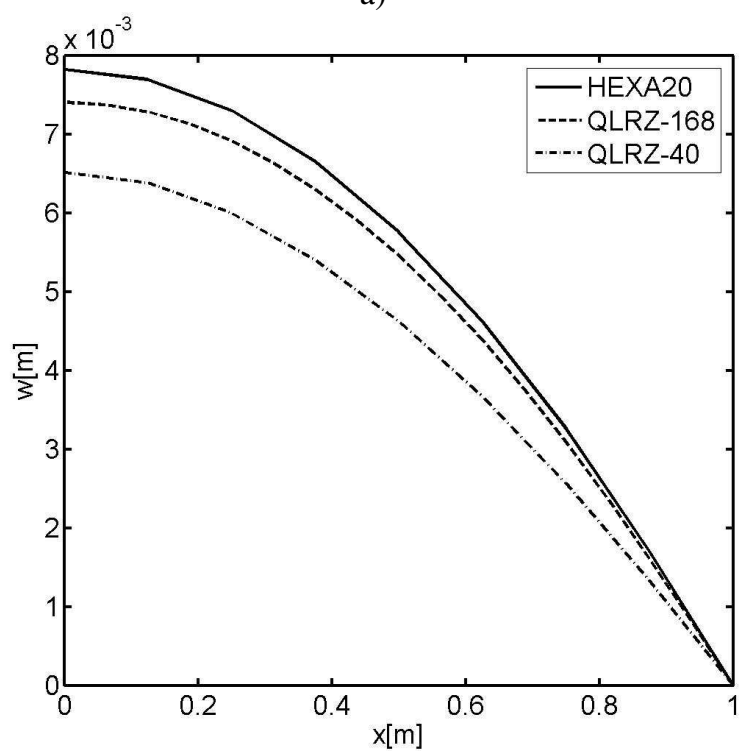

c)

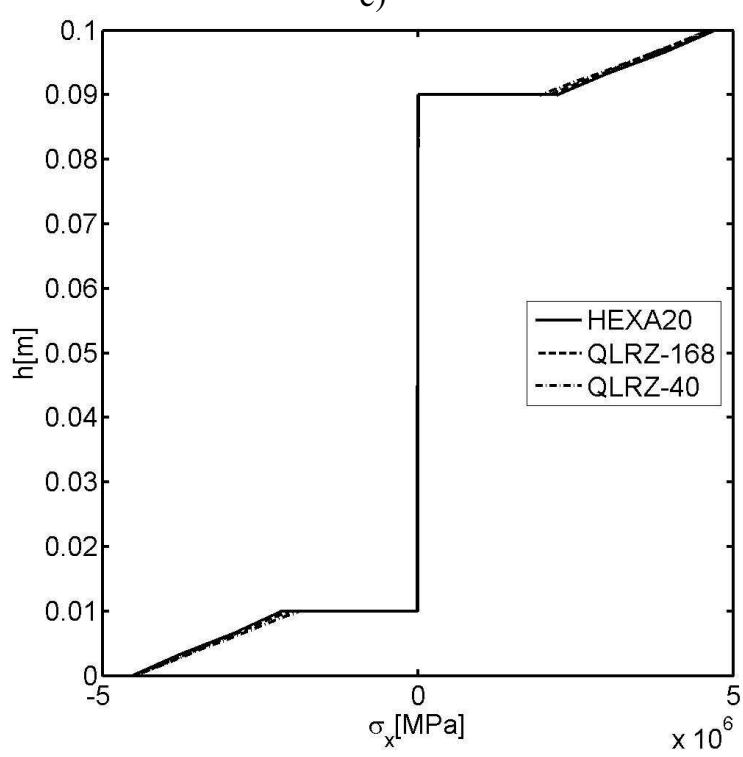

b)

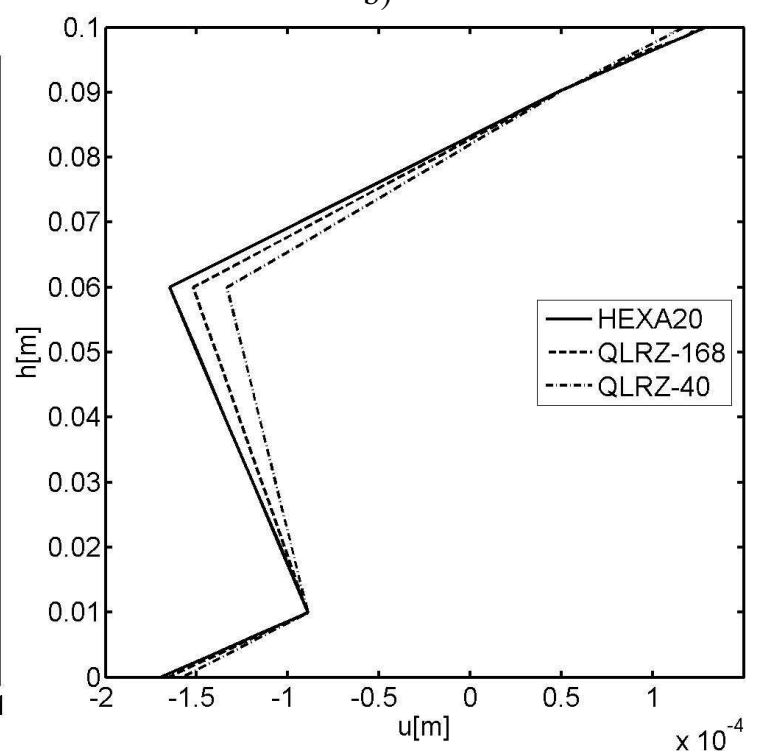

d)

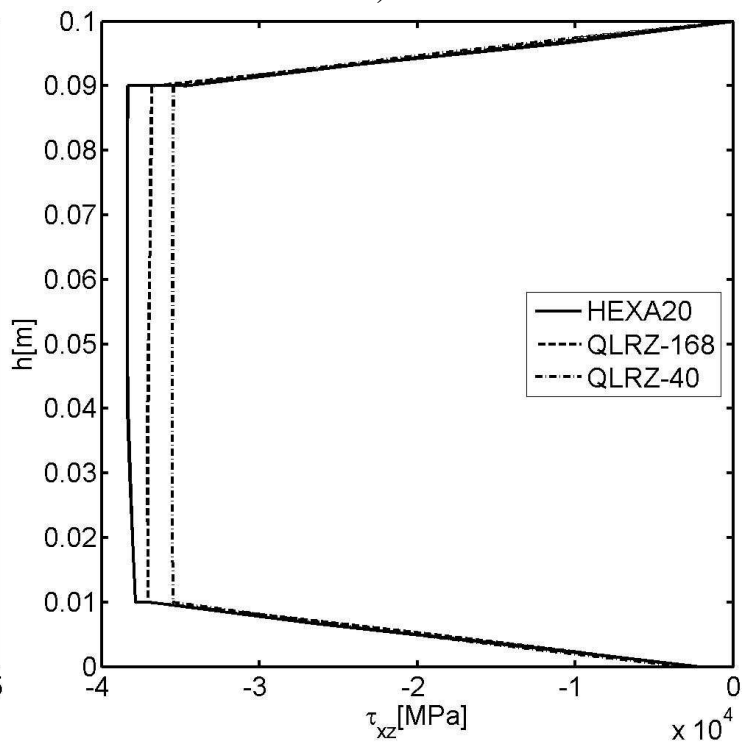

Figure 16 - SS circular plate under uniformly distributed load. Composite C6. a) Vertical deflection along line BC. Thickness distribution of: b) axial displacement $u$ at point $\mathrm{D}, \mathrm{c}$ ) axial stress $\sigma_{x}$ at the center point $\mathrm{C}$, and $\mathrm{d}$ ) transverse shear stress $\tau_{x z}$ at point $\mathrm{D}$. 
a)

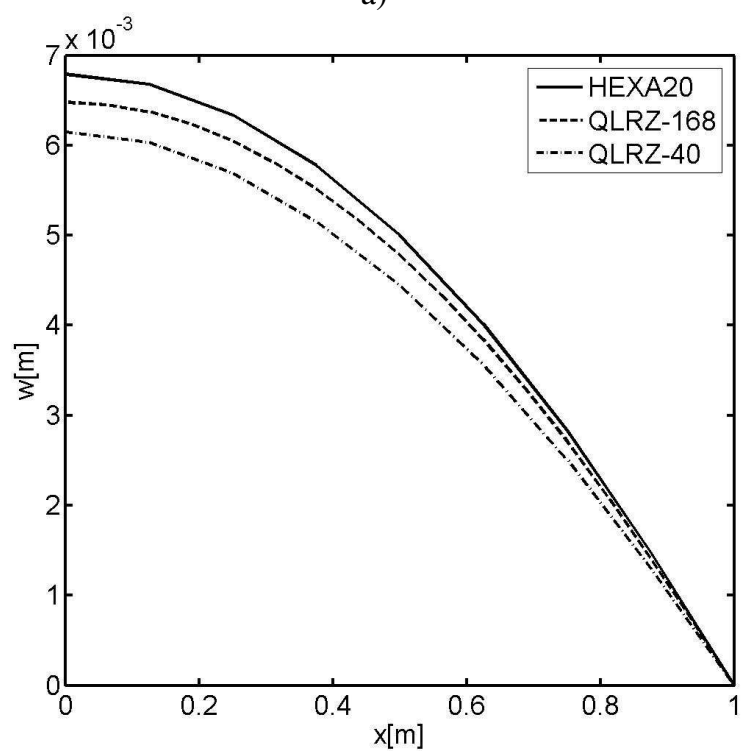

c)

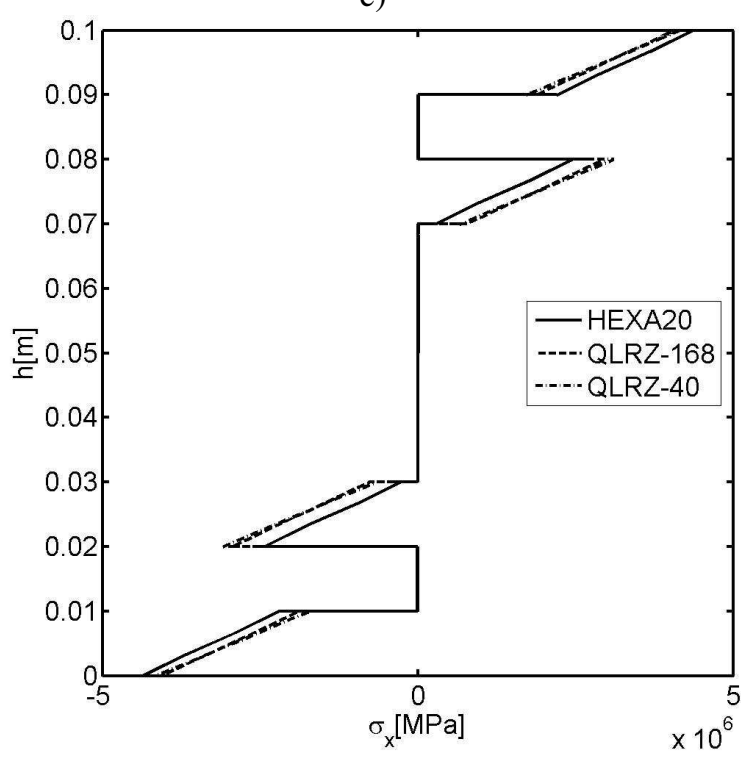

b)

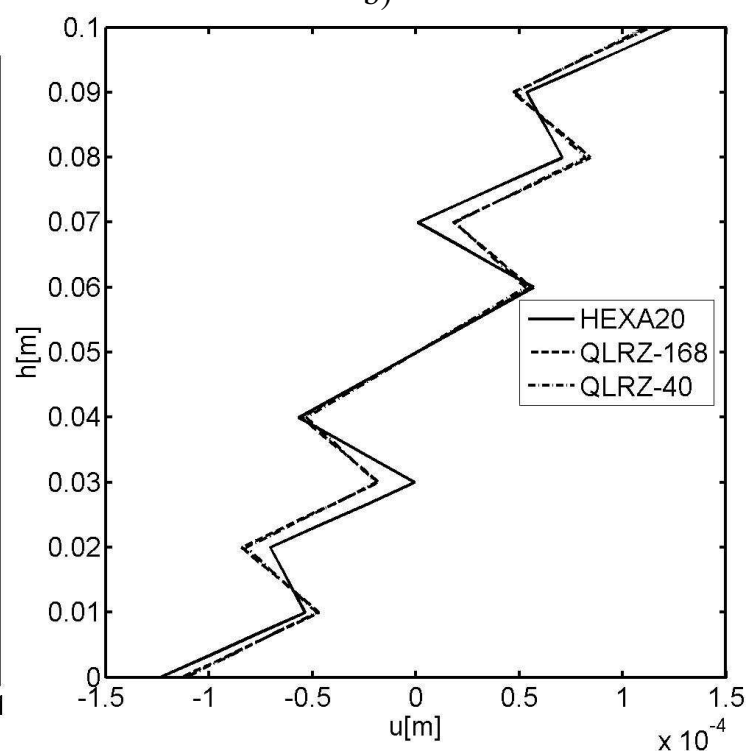

d)

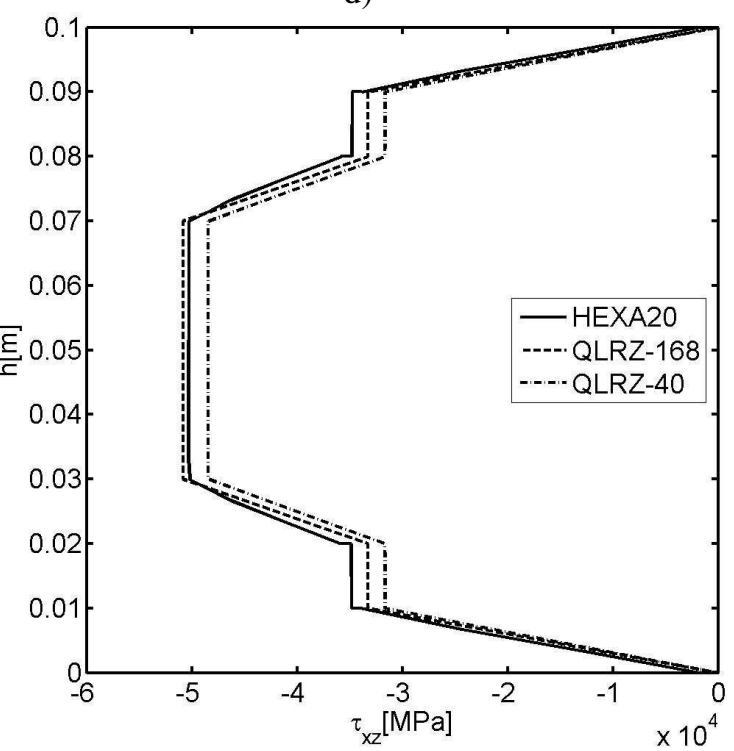

Figure 17 - SS circular plate under uniformly distributed load. Composite C7. a) Vertical deflection along line BC. Thickness distribution of: b) axial displacement $u$ at point $\mathrm{D}, \mathrm{c}$ ) axial stress $\sigma_{x}$ at the center point $\mathrm{C}$, and $\mathrm{d}$ ) transverse shear stress $\tau_{x z}$ at point $\mathrm{D}$.

\section{MODELING OF DELAMINATION WITH THE QLRZ ELEMENT}

Delamination, i.e. interlaminar cracks, is a common and dangerous source of damage in laminated composite materials [15], hence the higher interest of scientific community in its modeling. The simulation of delamination in plate and shell structures is still a challenger in computational solid mechanics. Among the most popular techniques to model the delamination phenomenon are the Virtual Crack Closure Technique (VCCT) [16], the Cohesive Finite Elements [17-19] and the Continuous Mechanics using damage models [20]. All of them need a "3D discretization" and, as mentioned above, this may lead to expensive computational costs for laminated composites of hundred of plies. Therefore, the use of plate models able to simulate delamination is a motivating alternative. 
In this section, the capability of the QLRZ element to model effectively delamination effects in laminated composite plates is shown. The delamination model chosen here simply implies introducing a very thin ply between adjacent material layers in the laminated composite section. Delamination occurs when the material properties of the added ply are reduced due to interlaminar failure by using a continuous damage model. The QLRZ element with this simple delamination model can take into account the reduction of the plate stiffness do to the interlaminar failure. Moreover, the QLRZ element can also accurately represent the jump in the axial displacement field across the layers.

Next we present an example where the SS square plate of Figure 9a ( $L=2 m$ and $h=0.1 \mathrm{~m})$ is analyzed under uniformly distributed load $q=10000 \mathrm{~N} / \mathrm{m}^{2}$. The $\mathrm{C} 7$ composite laminated material is employed (Table 9), and its material properties are shown in Table 8 . The objective is to simulate delamination between layers 3 and 4 by introducing a very thin layer $(h=0.001 \mathrm{~m})$ between these two layers (Figure 18), whose initial properties coincide with those of the layer 4 . The delamination is progressively induced by reducing the shear modulus of the interface layer up to 5 orders of magnitude from $G_{I L 1}=0.04 \times 10^{3} \mathrm{MPa}$ (model 1) to $G_{I L 6}=0.04 \times 10^{-2} \mathrm{MPa}$ (model 6) (Table 12). This reduction is applied over the whole plate surface. The QLRZ solution is obtained by using the $16 \times 16$ mesh of Figure 10. The reference solution employed is obtained by a 3D finite element analysis using a mesh of $8 \times 8 \times 28$ HEXA20 elements ( 3 elements per layers L1-9, and 1 element for the interface layer).

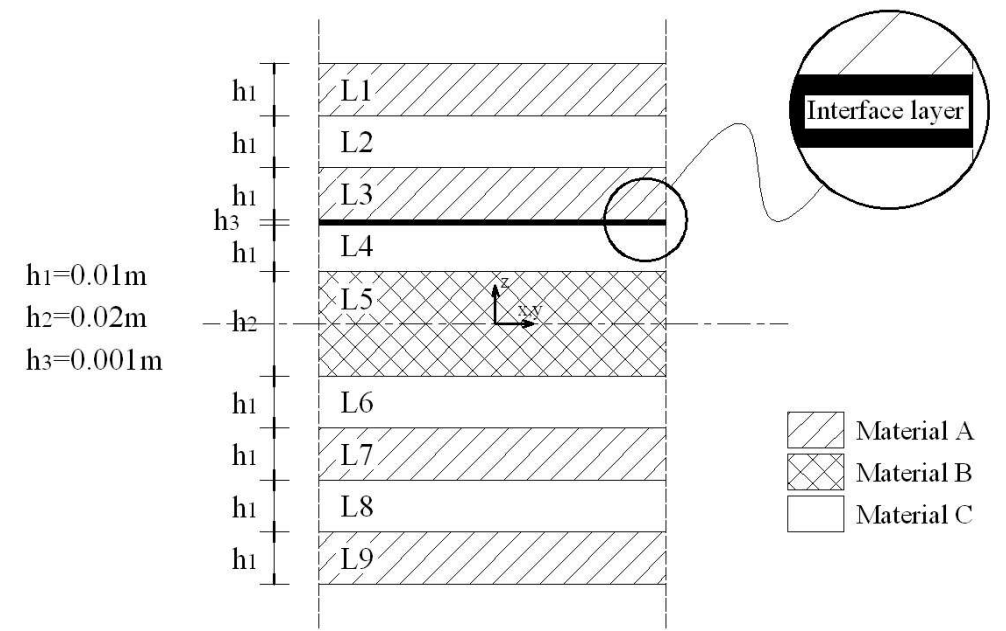

Figure 18 - Laminated composite C7 to which a thin interface layer between layers 3 and 4 is added.

\begin{tabular}{|c|c|c|c|}
\hline \multicolumn{4}{|c|}{ Shear modulus values for interface layer } \\
\hline Model & $\mathrm{G}_{\mathrm{IL}}[\mathrm{MPa}]$ & Model & $\mathrm{G}_{\mathrm{IL}}[\mathrm{MPa}]$ \\
\hline 1 & $0,04 \times 10^{3}$ & 4 & $0,04 \times 10^{0}$ \\
\hline 2 & $0,04 \times 10^{2}$ & 5 & $0,04 \times 10^{-1}$ \\
\hline 3 & $0,04 \times 10^{1}$ & 6 & $0,04 \times 10^{-2}$ \\
\hline
\end{tabular}

Table 12 - Induced shear modulus values of the interface layer for delamination study. 
Figure 19 shows the evolution of the vertical deflection at the plate center in terms of the shear modulus $G_{I L i}(i=1, \ldots, 6)$ of the interface layer (Table 12). Note that the deflection increases as the shear modulus decreases. Also, it is interesting that the deflection does not change after model $5\left(G_{I L 5}=0.04 \times 10^{-1} M P a\right)$. This may mean that after this value of $G_{I L 5}$ the composite $\mathrm{C} 7$ is separated into two completely disconnected parts (layers 1-3 and layers 4-9), and, therefore, is unable to transmit the shear stress across the section. The relative error for the central deflection, between the QLRZ and the HEXA20 solutions, is near to $25 \%$ for model 6.

Figure 20 shows the thickness distribution of the axial displacement along $x$ direction measured at point $\mathrm{E}$ (Figure 9a). As can be observed, the jump of axial displacement between layers 3 and 4 during delamination is well captured. Note that the jump remains stationary after model 5 according to the no change in the deflection value.

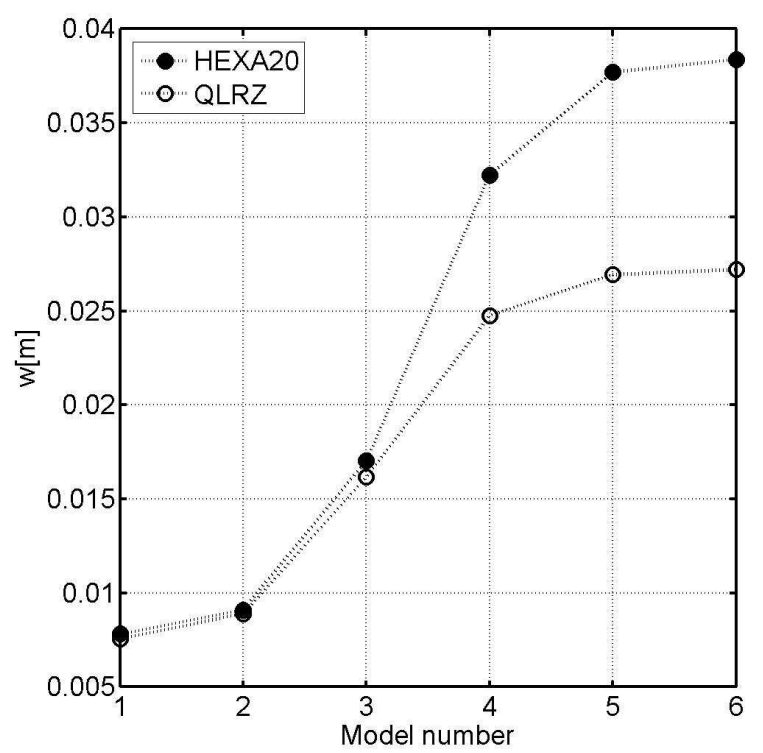

Figure 19 - Vertical deflection at center of plate for the different shear modulus values of the interface layer. 
a)

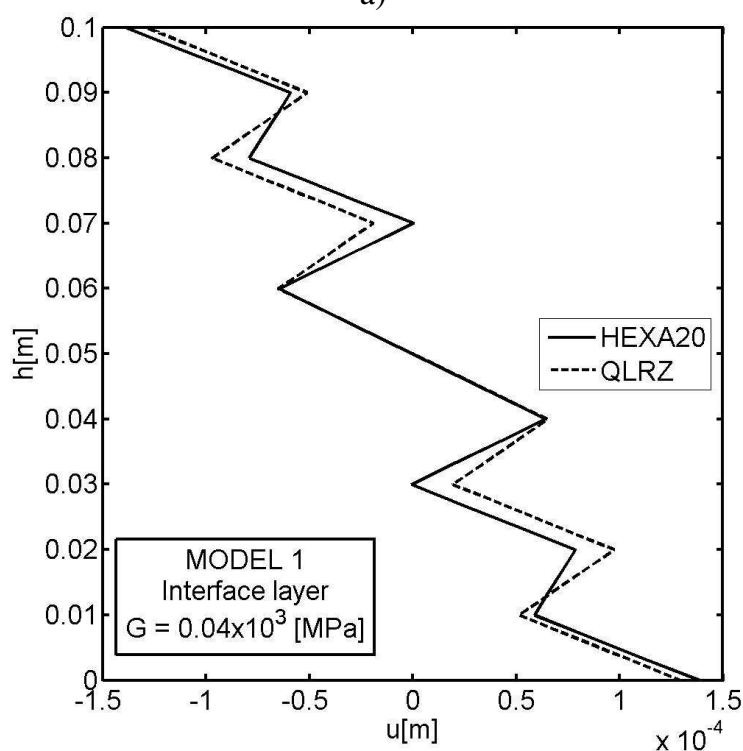

c)

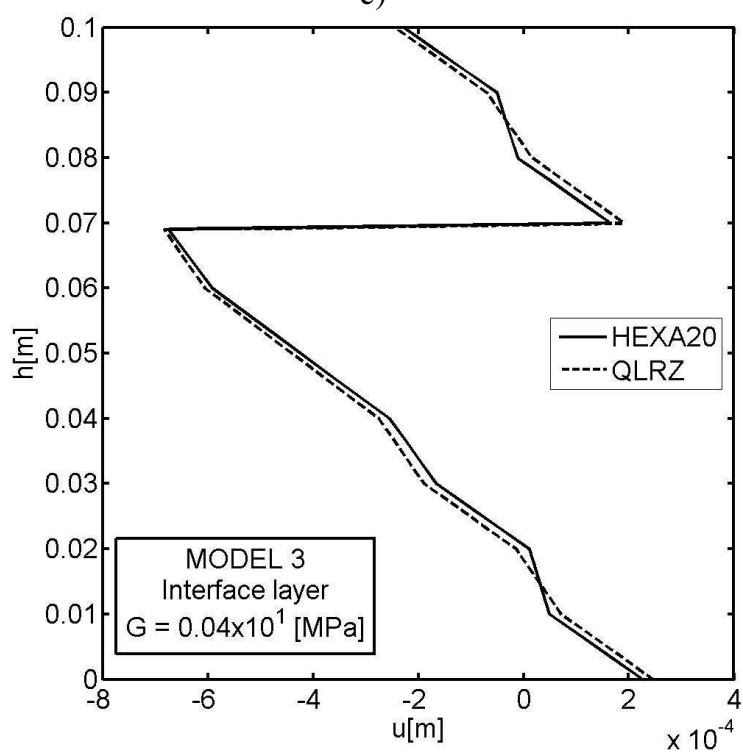

e)

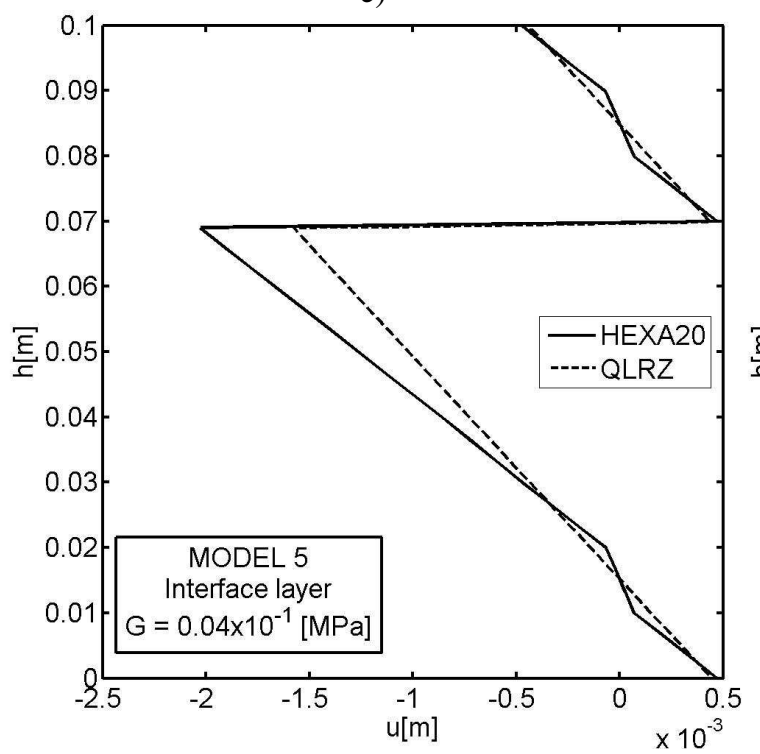

b)

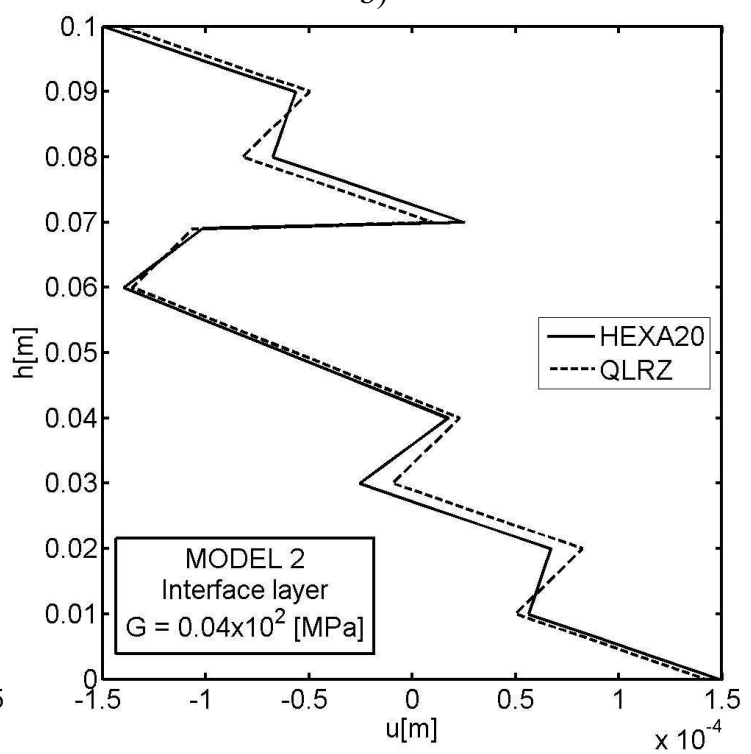

d)

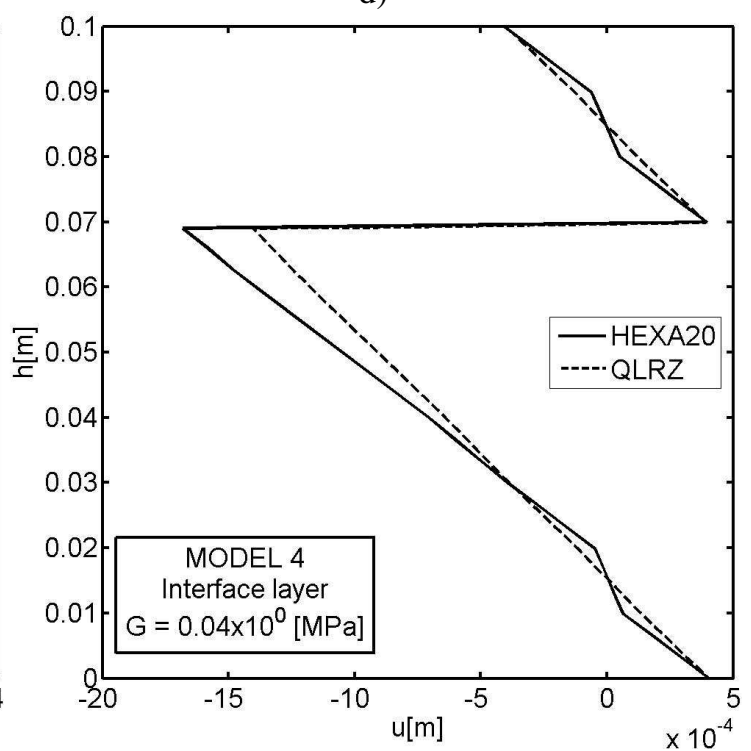

f)

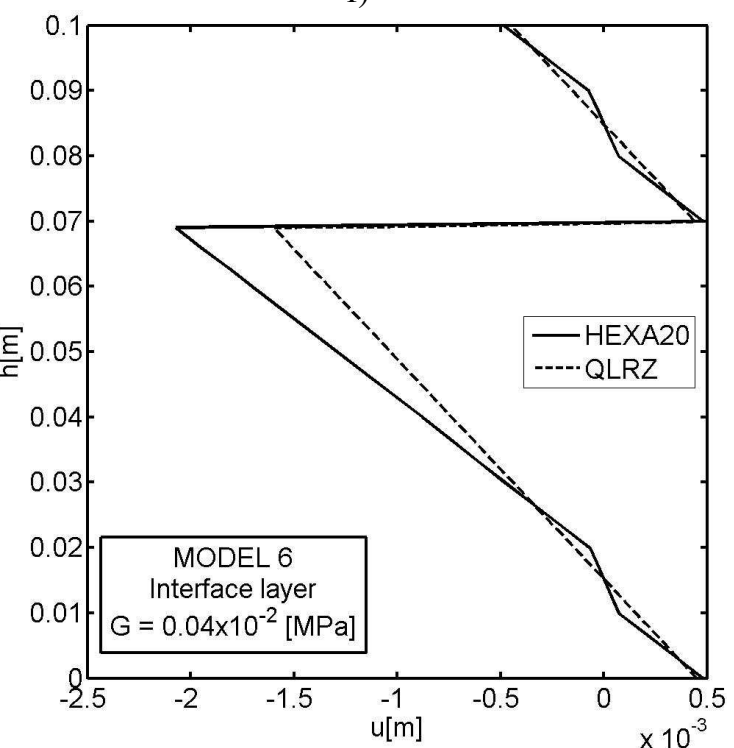

Figure 20 - Thickness distribution of axial displacement along $x$ direction at point $\mathrm{E}$ for each model. 


\section{CONCLUSIONS}

A simple, robust, shear locking free and accurate 4-noded plate element (called QLRZ) based on the refined zigzag theory has been presented. The shear locking defect was overcome by introducing an assumed linear shear strain field. The element has only seven unknowns per node which are interpolated by standard $\mathrm{C}^{0}$ linear shape functions. The thickness distribution of the transverse shear stresses is accurately reproduced by a posteriori computational process. The verification analysis has shown that the element is able to accurately model plates of homogeneous material for different loads and boundary conditions. The influence of the heterogeneity of composite laminated material on the convergence and accuracy of QLRZ solution has been studied. An important feature of the QLRZ element is its ability to capture the zigzag distribution of axial displacement and the subsequent complex strain and stress distribution across the thickness with the simple approximation chosen. This property makes possible to predict delamination effects as it has been shown in a simple demonstrative example.

\section{ACKNOWLEDGEMENTS}

This research was partially financial supported by the SAFECON project of the European Research Council (ERC) of the European Commission.

\section{ANNEX I}

\section{SHEAR LOCKING SOLUTION}

It is known that the standard four node plate element based on the Reissner-Mindlin theory (RMT) exhibits shear locking for thin plates [13]. Taking into account that the kinematic of RZT is a sum between the RMT and the zigzag displacement, we assume that the QLRZ element also suffers this defect. This assumption will be later evidenced in this section.

In the next we present two alternatives to overcome this drawback. The first is based on a reduced integration of the transverse stiffness matrix $\mathrm{K}_{t}$ (Eq.(20a)). The second is based on an assumed transverse shear strain field [13].

\section{AI.1 REDUCED INTEGRATION}

It is well known that shear locking can be eliminated by using reduced integration of the transverse shear stiffness matrix. Let us remember that $\mathrm{K}_{t}$ has second-order polynomials for rectangular elements, this means the exact integration is obtained using a $2 \times 2$ Gauss quadrature. The reduced integration implies using a 1x1 Gauss quadrature.

Let us retake the previous matrix $\mathrm{K}_{t}$ from Eq.(20a)

$$
\mathrm{K}_{t}=\mathrm{K}_{s}+\mathrm{K}_{s \phi}+\mathrm{K}_{s s \phi}+\left[\mathrm{K}_{s s \phi}\right]^{T}
$$

In order to assess the influence of the reduced integration of the matrix $\mathrm{K}_{t}$, the following integration combinations are selected. 


\begin{tabular}{|c|c|c|}
\hline \multicolumn{3}{|c|}{ INTEGRATION COMBINATIONS } \\
\hline Combinations & Exact & Reduced \\
\hline C1 & $\mathrm{K}_{s \phi} ; \mathrm{K}_{s s \phi}$ & $\mathrm{K}_{s}$ \\
\hline C2 & $\mathrm{K}_{s \phi}$ & $\mathrm{K}_{s} ; \mathrm{K}_{s s \phi}$ \\
\hline C3 & - & $\mathrm{K}_{t}$ \\
\hline
\end{tabular}

Table AI-1 - Integration combinations used to assess the influence of the reduced integration of $\mathrm{K}_{t}$.

The two key attributes of the reduced integration technique are, first, easy implementation in a finite element code, and second, a reduced computational cost. As a drawback, the reduced integration can originate new mechanisms (in addition to the rigid body motions) incompatible with the boundary conditions, which can propagate within the mesh. This problem is shown in the examples of section AI.3.

\section{AI.2 ASSUMED TRANSVERSE SHEAR STRAIN FIELDS}

A thin plate element must satisfy Kirchhoff condition of zero transverse shear strains, that is

$$
\varepsilon_{t}=\varepsilon_{s}+\varepsilon_{s \phi}=0
$$

where $\varepsilon_{s}$ and $\varepsilon_{s \phi}$ contain the average transverse shear strains of RMT ( $\gamma_{x z}$ and $\gamma_{y z}$ ) and the primary kinematic variables $\psi_{x}$ and $\psi_{y}$ (Eq.(1b)), respectively. Although the condition (AI.2) implies $\varepsilon_{s}+\varepsilon_{s \phi}=0$, we know that shear locking in a 4-noded ReissnerMindlin element is due to $\varepsilon_{s}$ [13], therefore, we consider that this effect in QLRZ elements is also due to the RMT transverse shear strain $\varepsilon_{s}$. Taking into account that $\varepsilon_{s}=\hat{\varepsilon}_{s}$, the condition (AI.2) can be reduced to

$$
\hat{\varepsilon}_{s}=0
$$

With the aim of demonstrate the origin of the locking effect in the QLRZ element, $\hat{\varepsilon}_{s}$ is explicitly developed by using Eqs.(16a) and (21). For simplicity, we consider $\gamma_{x z}$ only.

From Eq.(16a),

$$
\gamma_{x z}=\frac{\partial w_{0}}{\partial x}-\theta_{x}=\sum_{i=1}^{4} \frac{\partial N_{i}}{\partial x} \cdot w_{i}-N_{i} \cdot \theta_{x i}
$$

Substituting Eq.(16a) into Eq.(AI.4.a)

$$
\gamma_{x z}=\sum_{i=1}^{4}\left[\left(\frac{\xi_{i}}{4 a} w_{i}-\frac{1}{4} \theta_{x i}\right)+\left(\frac{\xi_{i} \eta_{i}}{4 a} w_{i}-\frac{\eta_{i}}{4} \theta_{x i}\right) \cdot \eta+\left(\frac{\xi_{i}}{4} \theta_{x i}\right) \cdot \xi-\left(\frac{\xi_{i} \eta_{i}}{4 a} \theta_{x i}\right) \cdot \xi \eta\right]
$$

Factoring Eq.(AI.4.b)

$$
\gamma_{x z}=\alpha_{1}\left(w_{i}, \theta_{x i}\right)+\alpha_{2}\left(w_{i}, \theta_{x i}\right) \cdot \eta+\alpha_{3}\left(\theta_{x i}\right) \cdot \xi+\alpha_{4}\left(\theta_{x i}\right) \cdot \xi \eta
$$

The Kirchhoff condition (AI.3) implies $\alpha_{i}=0(i=1,2,3,4) . \alpha_{1}=0$ and $\alpha_{2}=0$ are physically possible and they impose a relationship between $\theta_{x}$ and $w$. However, the element is unable to satisfy naturally the conditions $\alpha_{3}=0$ and $\alpha_{4}=0$, unless $\theta_{x i}=0$, which leads to $w_{i}=0$ (shear locking effect). Identical conclusion can be found for $\gamma_{y z}$ simply by replacing $\xi$ by $\eta$ and $\theta_{x i}$ by $\theta_{y i}$. 
Hence, it is possible to avoid the shear locking defect by imposing a transverse shear strain field (figure AI-1) like

$$
\hat{\varepsilon}_{s}=\left[\begin{array}{l}
\gamma_{x z} \\
\gamma_{y z}
\end{array}\right]=\left[\begin{array}{l}
\alpha_{1}\left(w_{i}, \theta_{x i}\right)+\alpha_{2}\left(w_{i}, \theta_{x i}\right) \cdot \eta \\
\alpha_{3}\left(w_{i}, \theta_{y i}\right)+\alpha_{4}\left(w_{i}, \theta_{y i}\right) \cdot \eta
\end{array}\right]=\overline{\mathrm{B}}_{s} \cdot \mathrm{a}^{(e)}
$$

where $\overline{\mathrm{B}}_{s}$ is the substitutive shear strain generalized matrix. This matrix is used instead of the original $\mathrm{B}_{s}$ for computing the shear stiffness matrices $\mathrm{K}_{s}$ and $\mathrm{K}_{s s \phi}$ of Eq.(20b), i.e.

$$
\begin{aligned}
& \mathrm{K}_{s}=\iint_{A} \overline{\mathrm{B}}_{s}{ }^{T} \cdot \hat{D}_{s} \cdot \overline{\mathrm{B}}_{s} \cdot d A \\
& \mathrm{~K}_{s s \phi}=\iint_{A} \overline{\mathrm{B}}_{s}{ }^{T} \cdot \hat{D}_{s s \phi} \cdot \mathrm{B}_{s \phi} \cdot d A
\end{aligned}
$$

These matrices can now be integrated exactly using a $2 \times 2$ Gauss quadrature.

Summarizing, the idea of this technique is to impose "a priori" a transverse shear strain field, which allowing the vanishing of $\hat{\varepsilon}_{s}$ in the thin limit. The assumed transverse shear strain interpolation is

$$
\hat{\varepsilon}_{s}=\sum_{k=1}^{m} \mathrm{~N}_{\gamma k} \cdot \gamma_{k}=\mathrm{N}_{\gamma} \cdot \gamma^{(e)}
$$

where $\gamma_{k}$ are the average transverse shear strain values at $m$ points within the element and $\mathrm{N}_{\gamma k}$ are the shear interpolating functions. By combining Eqs. (16a)and (AI.7.a) is obtained

$$
\hat{\varepsilon}_{s}=\sum_{k=1}^{m} \mathrm{~N}_{\gamma k} \cdot \mathrm{B}_{s k} \cdot \mathrm{a}_{k}^{(e)}=\overline{\mathrm{B}}_{s} \cdot \mathrm{a}^{(e)}
$$

and the new interpolations are

$$
\mathrm{a}=\mathrm{N} \cdot \mathrm{a}^{(e)} \quad ; \quad \hat{\mathcal{E}}_{s}=\mathrm{N}_{\gamma} \cdot \gamma^{(e)}
$$

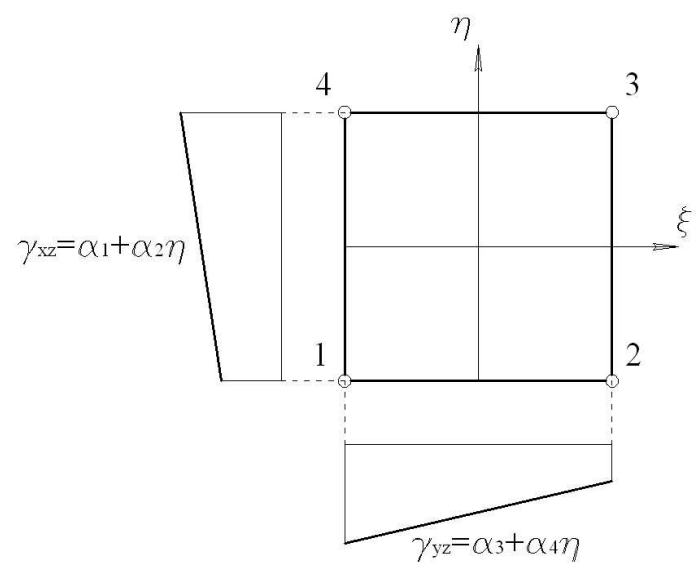

Figure AI-1 - Imposed transverse shear strain field.

AI.2.1 Computation of substitutive shear strain generalized matrix $\bar{B}_{s}$

The natural transverse shear strain field is given by $[13,14]$

$$
\hat{\varepsilon}_{s}^{\prime}=\left[\begin{array}{l}
\gamma_{\xi} \\
\gamma_{\eta}
\end{array}\right]=\left[\begin{array}{l}
\alpha_{1}+\alpha_{2} \eta \\
\alpha_{3}+\alpha_{4} \xi
\end{array}\right]=\left[\begin{array}{llll}
1 & \eta & 0 & 0 \\
0 & 0 & 1 & \xi
\end{array}\right] \cdot\left[\begin{array}{l}
\alpha_{1} \\
\alpha_{2} \\
\alpha_{3} \\
\alpha_{4}
\end{array}\right]=\mathrm{A} \cdot \alpha
$$


The transverse shear strains $\hat{\varepsilon}_{s}$ in the cartesian coordinate system are expressed as

$$
\hat{\varepsilon}_{s}=\left[\begin{array}{l}
\gamma_{x z} \\
\gamma_{y z}
\end{array}\right]=\mathrm{J}^{-1} \cdot \hat{\varepsilon}_{s}^{\prime}
$$

where $\mathbf{J}$ is the $2 \mathrm{D}$ Jacobian matrix

$$
\mathrm{J}=\left[\begin{array}{ll}
\frac{\partial x}{\partial \xi} & \frac{\partial y}{\partial \xi} \\
\frac{\partial x}{\partial \eta} & \frac{\partial y}{\partial \eta}
\end{array}\right]
$$

The coefficients $\alpha_{i}$ are obtained by sampling the natural shear strains (Eq.(AI.9)) at the four points shown in Figure AI-2, with

$$
\gamma_{\xi_{i}}=\left(\alpha_{1}+\alpha_{2} \eta\right) \cdot \cos \delta_{i}+\left(\alpha_{3}+\alpha_{4} \xi\right) \cdot \sin \delta_{i} \quad ; \quad i=1,4
$$

where $\delta_{i}$ is the angle between $\bar{\xi}_{i}$ direction and the natural $\xi$ axis. Combining Eqs. (AI.9) and (AI.11) gives

$$
\begin{gathered}
\gamma_{\bar{\xi}}=\left[\begin{array}{c}
\gamma_{\bar{\xi}_{1}} \\
\gamma_{\bar{\xi}_{2}} \\
\gamma_{\bar{\xi}_{3}} \\
\gamma_{\bar{\xi}_{4}}
\end{array}\right]=\left[\begin{array}{cccc}
1 & -1 & 0 & 0 \\
0 & 0 & 1 & 1 \\
1 & 1 & 0 & 0 \\
0 & 0 & 1 & -1
\end{array}\right] \cdot\left[\begin{array}{l}
\alpha_{1} \\
\alpha_{2} \\
\alpha_{3} \\
\alpha_{4}
\end{array}\right]=\mathrm{P} \cdot \alpha \\
\alpha=\mathrm{P}^{-1} \cdot \gamma_{\bar{\xi}}
\end{gathered}
$$

where the strains $\gamma_{\xi_{i}}$ are related to $\gamma_{\xi_{i}}$ and $\gamma_{\eta_{i}}$ by

$$
\gamma_{\bar{\xi}}=\left[\begin{array}{c}
\gamma_{\xi_{1}} \\
\gamma_{\bar{\xi}_{2}} \\
\gamma_{\bar{\xi}_{3}} \\
\gamma_{\xi_{4}}
\end{array}\right]=\left[\begin{array}{llllllll}
1 & 0 & 0 & 0 & 0 & 0 & 0 & 0 \\
0 & 0 & 0 & 1 & 0 & 0 & 0 & 0 \\
0 & 0 & 0 & 0 & 1 & 0 & 0 & 0 \\
0 & 0 & 0 & 0 & 0 & 0 & 0 & 1
\end{array}\right] \cdot\left[\begin{array}{l}
\gamma_{\xi_{1}} \\
\gamma_{\eta_{1}} \\
\gamma_{\xi_{2}} \\
\gamma_{\eta_{2}} \\
\gamma_{\xi_{3}} \\
\gamma_{\eta_{3}} \\
\gamma_{\xi_{4}} \\
\gamma_{\eta_{4}}
\end{array}\right]=\mathrm{T} \cdot \hat{\gamma}
$$

Combining Eqs. (AI.9), (AI.12) and (AI.13) gives

$$
\hat{\varepsilon}_{s}^{\prime}=\mathrm{A} \cdot \mathrm{P}^{-1} \cdot \mathrm{T} \cdot \hat{\gamma}^{\prime}
$$

The cartesian transverse shear strains $\hat{\gamma}$ at the sampling points are related to the natural transverse shear strains $\hat{\gamma}$ by

$$
\hat{\gamma}^{\prime}=\left[\begin{array}{cccc}
\mathbf{J}^{1} & 0 & 0 & 0 \\
0 & \mathbf{J}^{2} & 0 & 0 \\
0 & 0 & \mathbf{J}^{3} & 0 \\
0 & 0 & 0 & \mathbf{J}^{4}
\end{array}\right] \cdot\left[\begin{array}{l}
\hat{\gamma}_{1} \\
\hat{\gamma}_{2} \\
\hat{\gamma}_{3} \\
\hat{\gamma}_{4}
\end{array}\right]=\mathrm{C} \cdot \hat{\gamma} \quad ; \quad \hat{\gamma}_{i}=\left[\begin{array}{l}
\gamma_{x z} \\
\gamma_{y z}
\end{array}\right]_{i}
$$

The relationship between the cartesian shear strains $\hat{\gamma}$ at the four sampling points (figure AI-2) and the nodal displacements $\mathrm{a}^{(e)}$ is 


$$
\hat{\gamma}=\mathrm{B}_{s} \cdot \mathrm{a}^{(e)}
$$

with

$$
\mathrm{B}_{s}=\left[\begin{array}{llll}
B_{s 1} & B_{s 2} & B_{s 3} & B_{s 4}
\end{array}\right]^{T}
$$

where $B_{s i}(i=1,2,3,4)$ is the original transverse generalized strain matrix (Eq.(16c)) at the $i$ th sampling point.

Combining Eqs. (AI.10.a), (AI.14), (AI.15) and (AI.16.a) gives

$$
\hat{\varepsilon}_{s}=\mathrm{J}^{-1} \cdot \mathrm{A} \cdot \mathrm{P}^{-1} \cdot \mathrm{T} \cdot \mathrm{C} \cdot \mathrm{B}_{s} \cdot \mathrm{a}=\overline{\mathrm{B}}_{s} \cdot \mathrm{a}
$$

where $\overline{\mathrm{B}}_{s}$ is the sought substitute transverse shear strain matrix given by

$$
\overline{\mathrm{B}}_{s}=\mathrm{J}^{-1} \cdot \mathrm{A} \cdot \mathrm{P}^{-1} \cdot \mathrm{T} \cdot \mathrm{C} \cdot \mathrm{B}_{s}
$$
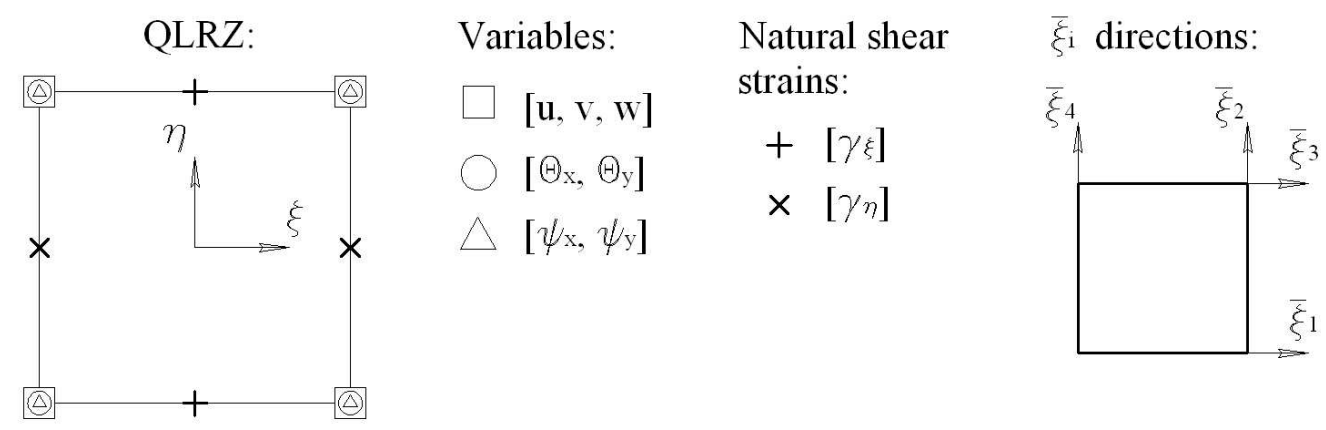

Figure AI-2 - QLRZ plate element.

\section{AI.3 STUDY OF ALTERNATIVES}

In order to show the efficacy of the two selected alternative and to show the mechanisms creation when the reduced integrations of Table AI-1 are used, are analyzed tow case.

A simply supported square plate of length side $L=2$ under a uniformly distributed load of unit value $(q=1)$ is analyzed in the first example (Figure AI-3a). The analysis is performed for four span-to-thickness ratios: $\lambda=L / h=5,10,50,100$. A clamped square plate of length side $L=2$ and thickness $h=0.2(\lambda=10)$ under a center point load of value $P=4$ is studied in the second example (Figure AI-3b). Both structures have using a composite laminated material whose properties are listed in Table AI-2. Only one quarter of the plate is studied due to symmetry (Figure AI-3) using a mesh of 16x16 QLRZ elements (Figure AI-4a) whit 289 nodes and 1445 DOFs. The reference solution is obtained by a 3D finite element analysis using a mesh of 10x10x9 (3 elements per ply) of 20-noded hexahedral elements (HEXA20) involving 4499 nodes and 13497 DOFs (Figure AI-4b). 


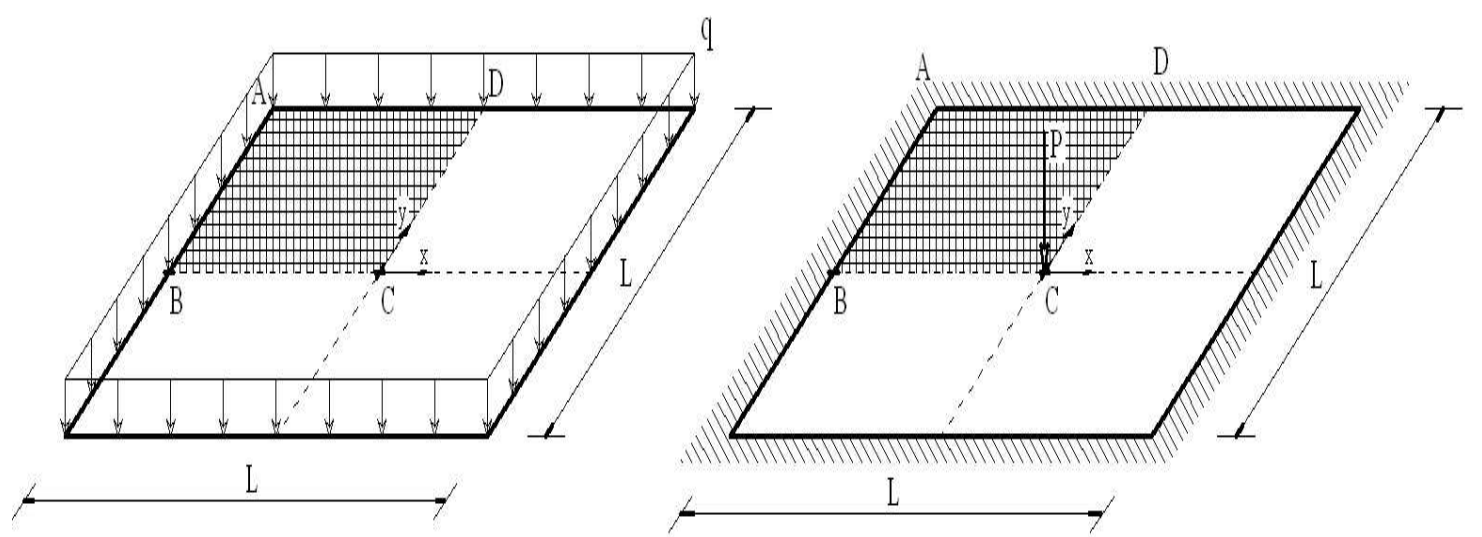

Figure AI-3 - Simply supported square plate under uniformly distributed load a). Clamped square plate under center point load b).

\begin{tabular}{|c|c|c|c|}
\hline \multicolumn{4}{|c|}{ Composite material properties } \\
\hline Properties & $\begin{array}{c}\text { Layer 1 } \\
\text { (top) }\end{array}$ & Layer 2 & $\begin{array}{c}\text { Layer 3 } \\
\text { (bottom) }\end{array}$ \\
\hline h & $0,25 \cdot L / \lambda$ & $0,50 \cdot L / \lambda$ & $0,25 \cdot L / \lambda$ \\
\hline E & $2,19 \mathrm{E} 5$ & $2,19 \mathrm{E} 4$ & $4,4 \mathrm{E} 5$ \\
\hline G & $0,876 \mathrm{E} 5$ & $0,876 \mathrm{E} 4$ & $1,76 \mathrm{E} 5$ \\
\hline
\end{tabular}

Table AI-2 - Material properties for shear locking study.

a)

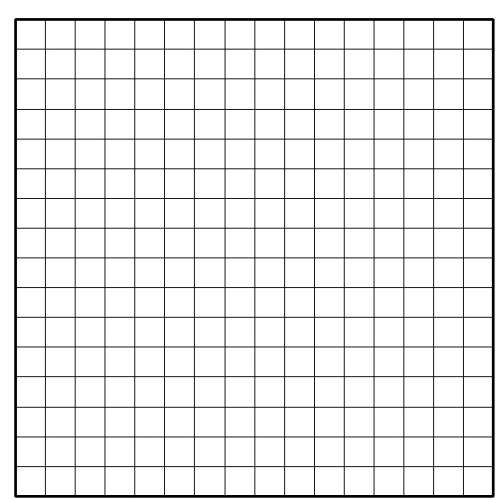

b)

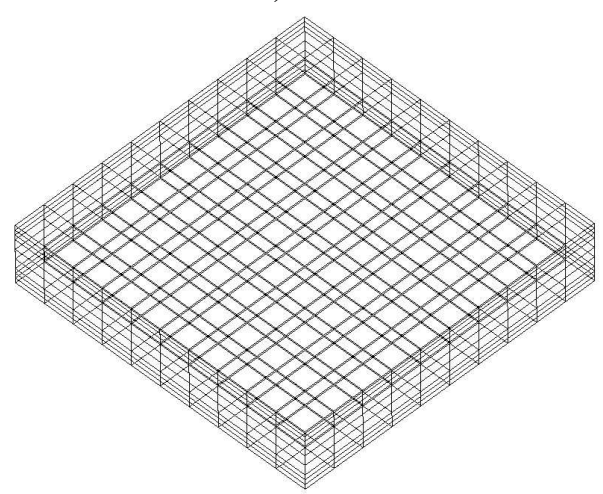

$\mathbf{V}_{x}^{2}$

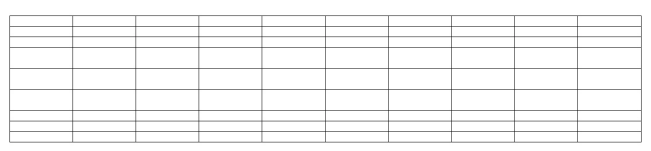

Figure AI-4 - Meshes used for the analysis of one quarter of the SS plate. 16x16 QLRZ elements a) and 10x10x9 HEXA20 elements b).

Figure AI-4 shows the $r$ ratio defined as

$$
r=\frac{w_{Q L R Z}}{w_{3 D}}
$$


where $w_{Q L R Z}$ and $w_{3 D}$ are the middle deflection at the plate center obtained with the QLRZ element and the 3D finite element analysis, respectively. The QLRZ element results have been obtained using exact integration of matrix $\mathrm{K}_{t}$ (exact), a reduced integration of matrices $\mathrm{K}_{s}, \mathrm{~K}_{s \phi}$ and $\mathrm{K}_{s s \phi}$ for the three combinations of table AI-1 (C1, $\mathrm{C} 2$, and $\mathrm{C} 3$ ), and finally using the assumed transverse shear strain field technique (QLRZ).

(a)

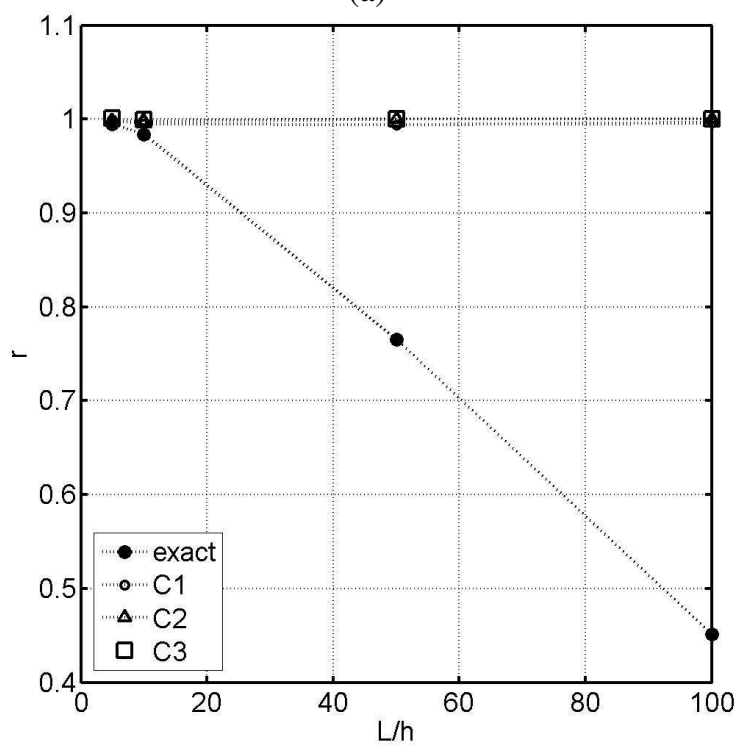

(b)

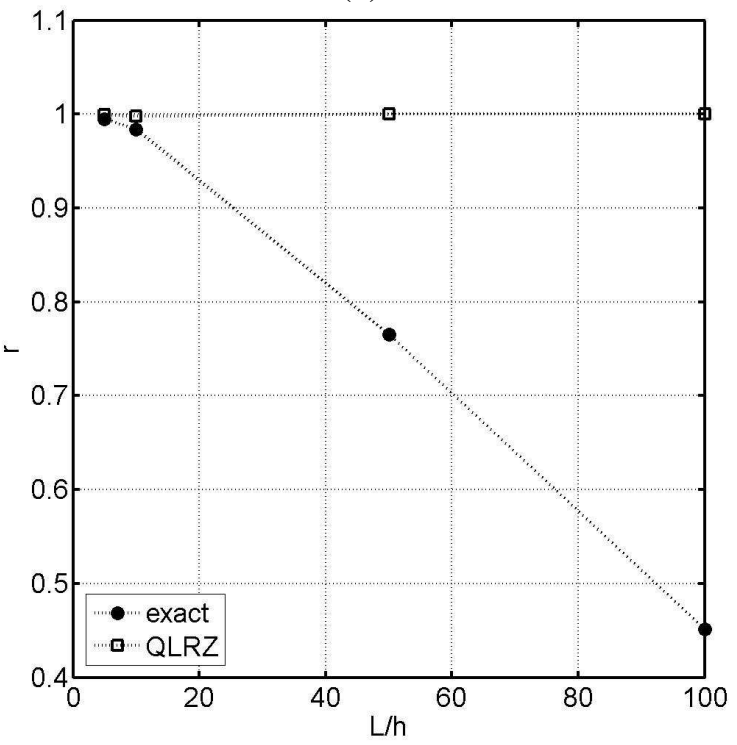

Figure AI-5 - $r$ ratio vs. span-to-thickness $\lambda$. Simply supported square plate under uniformly distributed load. Figure a): exact integration (exact) and the three integration combinations (C1, C2, and C3) of table AI-1. Figure b): exact integration and assumed transverse shear strain fields (QLRZ).

Figure AI-5 clearly shows the shear locking defects when exact integration of $\mathbf{K}_{t}^{(e)}$ is used. However, this defect disappears by using both techniques.

Figure AI-6 shows the distribution of the vertical deflection $w$ along the plate central line $\mathrm{BC}$ (Figure AI-3b) obtained with exact integration of matrix $\mathrm{K}_{t}$, by using reduced integration (Table AI-1) and assumed transverse shear strain technique, and 3D analysis (HEXA20). Figure AI-6a reveals the existence of mechanisms in mesh when reduced integration is used. These mechanisms do not appear if the assumed transverse shear strain technique is used. 
(a)

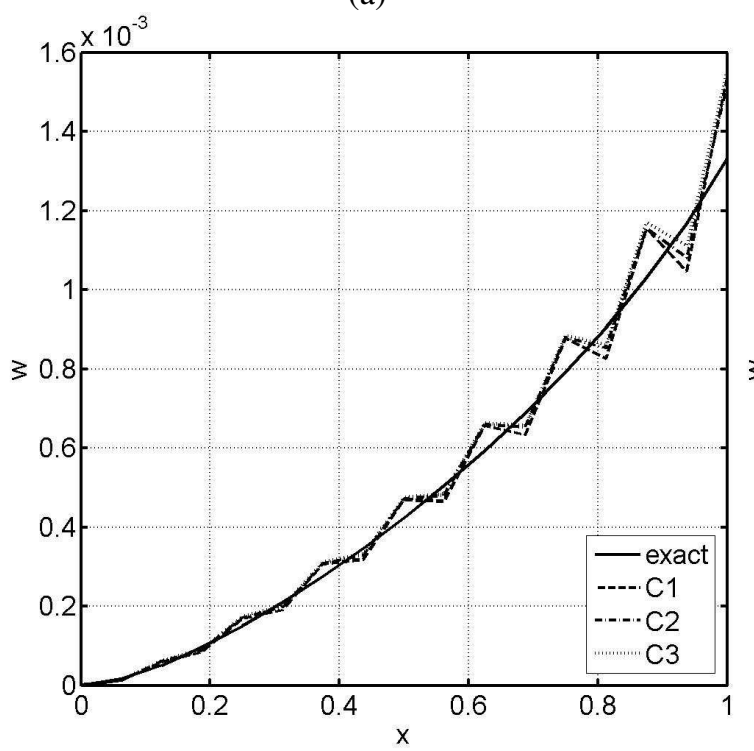

(b)

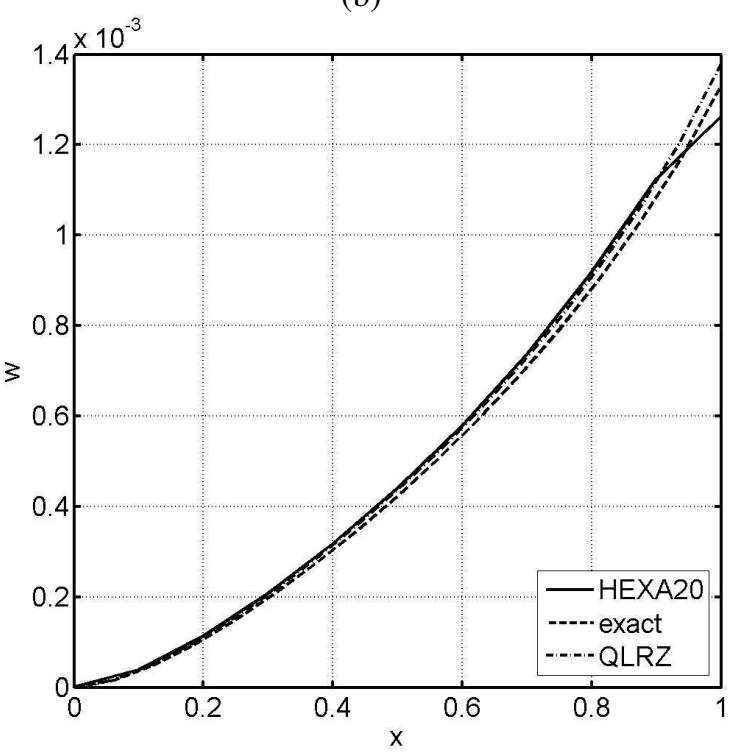

Figure AI-6 - Vertical deflection $w$ along BC. Clamped square plate $(\lambda=10)$ under a center point load. Figure a): exact integration (exact) and the three integration combinations (C1, C2, and C3) of table AI-1. Figure b): exact integration, assumed transverse shear strain fields (QLRZ), and 3D analysis (HEXA20).

The results show that the assumed transverse shear strain technique is adequate to develop a robust shear locking free plate element.

\section{REFERENCES}

[1] Tessler, A., Sciuva, M. D., and Gherlone, M., A consistent refinement of first-order shear deformation theory for laminated composite and sandwich plates using improved zigzag kinematics, Mechanics of Materials and Structures, 2010, Vol. 5(2), pp. 341-365.

[2] Kirchhoff, G., Über das Gleichgewicht und die Bewegung einer elastishen Scheibe, J Angew Math, 1850, Vol. 40(-), pp. 51-88.

[3] Reissner, E., The effect of transverse shear deformation on the bending of elastic plates, Appl. Mech., 1945, Vol. 12(-), pp. 69-79.

[4] Mindlin, R. D., Influence of rotatory inertia and shear in flexural motions of isotropic elastic plates, Appl. Mech., 1951, Vol. 18(-), pp. 31-38.

[5] Liu, D. and Li, X., An overall view of laminate theories based on displacement hypothesis, Journal of Composite Materials, 1996, Vol. 30(14), pp. 1539-1561.

[6] Wanji, C. and Zhen, W., A Selective Review on Recent Development of Displacement-Based Laminated Plate Theories, Recent Patents on Mechanical Engineering, 2008, Vol. 1(-), pp. 2944.

[7] J. N. Reddy and D. H. Robbins, J., Theories and computational models for composite laminates, Applied Mechanics Reviews, 1994, Vol. 47(6), pp. 147-165.

[8] Carrera, E., Historical review of Zig-Zag theories for multilayered plates and shells, Applied Mechanics Reviews, 2003, Vol. 56(3), pp. 287-308.

[9] Tessler, A., Sciuva, M. D., and Gherlone, M., Refinement of Timoshenko Beam Theory for Composite and Sandwich Beams Using Zigzag Kinematics, Technical Publication TP-215086, NASA, 2007.

[10] Oñate, E., Eijo, A., and Oller, S., Simple and accurate two-noded beam element for composite laminated beams using a refined zigzag theory., Computer Methods in Applied Mechanics and Engineering, 2012, pp. 362-382.

[11] Gherlone, M., Tessler, A., and Di Sciuva, M., C ${ }^{\circ}$ beam element based on the refined zigzag theory for multilayered composite and sandwich laminates, Composite Structures. Accepted for publication, 2011

[12] Oñate, E., Eijo, A., and Oller, S., Two-noded beam element for composite and sandwich beams using Timoshenko theory and Refined ZigZag Kinematics, Publication No-346, CIMNE, 2010.

[13] Onate, E., Structural analysis by the finite element method, Vol. 2: Beams, plates and shells.CIMNE-Springer, Barcelona, 2012. 
[14] Oñate, E., Zienkiewicz, O., Suárez, B., and Taylor, R. L., A general methodology for deriving shear constrained Reissner-Mindlin plate elements, Int. Journal for Numerical Methods in Engineering, 1992, Vol. 33(2), pp. 345-367.

[15] Bolotin, V. V., Delaminations in composite structures: its origin, buckling, growth and stability., Composites: Part B 27B, 1996, pp. 129-145.

[16] Krueger, R., The Virtual Crack Closure Technique: History, Approach and Applications, Applied Mechanics Reviews, 2002, Vol. 57(2), pp. 109-143.

[17] Borg, R., Nilsson, L., and Simonsson, K., Modeling of delamination using a discretized cohesive zone and damage formulation, Composites Science and Technology, 2002, Vol. 62(10-11), pp. 1299-1314.

[18] Turon, A., Camanho, P. P., Costa, J., and Da'vila, C. G., A damage model for the simulation of delamination in advanced composites under variable-mode loading, Mechanics of Materials, 2006, Vol. 38(11), pp. 1072-1089.

[19] Balzani, C. and Wagner, W., An interface element for the simulation of delamination in unidirectional fiber-reinforced composite laminates, Engineering Fracture Mechanics, 2008, Vol. 75(9), pp. 2597-2615.

[20] Martinez, X., Rastellini, F., Oller, S., Flores, F., and Oñate, E., Computationally optimized formulation for the simulation of composite materials and delamination failures, Composites: Part B, 2011, Vol. 47(134-144. 